\title{
On the multi-orbital band structure and itinerant magnetism of iron-based superconductors
}

\author{
Ole Krogh Andersen ${ }^{1}$ and Lilia Boeri ${ }^{1}$
}

Key words Fe-based superconductors, Electronic Structure, Tight-Binding, Downfolding, Magnetism, Interlayer Coupling

This paper explains the multi-orbital band structures and itinerant magnetism of the iron-pnictide and chalcogenide superconductors. We first describe the generic band structure of a single, isolated FeAs layer. Use of its Abelian glide-mirror group allows us to reduce the primitive cell to one FeAs unit. For the lines and points of high symmetry in the corresponding large, square Brillouin zone, we specify how the one-electron Hamiltonian factorizes. From density-functional theory, and for the observed structure of $\mathrm{LaOFeAs}$, we generate the set of eight $\mathrm{Fe} d$ and As $p$ localized Wannier functions and their tight-binding (TB) Hamiltonian, $h(\mathbf{k})$. For comparison, we generate the set of five Fe $d$ Wannier orbitals. The topology of the bands, i.e. allowed and avoided crossings, specifically the origin of the $d^{6}$ pseudogap, is discussed, and the role of the As $p$ orbitals and the elongation of the FeAs 4 tetrahedron emphasized. We then couple the layers, mainly via interlayer hopping between As $p_{z}$ orbitals, and give the formalism for simple tetragonal and body-centered tetragonal (bct) stackings. This allows us to explain the material-specific 3D band structures, in particular the complicated ones of bct $\mathrm{BaFe}_{2} \mathrm{As}_{2}$ and $\mathrm{CaFe}_{2} \mathrm{As}_{2}$ whose interlayer hoppings are large. Due to the high symmetry, several level inversions take place as functions of $k_{z}$ or pressure, and linear band dispersions (Dirac cones) are found at many places. The underlying symmetry elements are, however, easily broken by phonons or impurities, for instance, so that the Dirac points are not protected. Nor are they pinned to the Fermi level because the Fermi surface has several sheets. From the paramagnetic TB Hamiltonian, we form the band structures for spin spirals with wavevector $\mathbf{q}$ by coupling $h(\mathbf{k})$ and $h(\mathbf{k}+\mathbf{q})$. The band structure for stripe order is studied in detail as a function of the exchange potential, $\Delta$, or moment, $m$, using Stoner theory. Gapping of the Fermi surface (FS) for small $\Delta$ requires matching of FS dimensions (nesting) and $d$-orbital characters. The interplay between $p d$ hybridization and magnetism is discussed using simple $4 \times 4$ Hamiltonians. The origin of the propeller-shaped Fermi surface is explained in detail. Finally, we express the magnetic energy as the sum over band-structure energies and this enables us to understand to what extent the magnetic energies might be described by a Heisenberg Hamiltonian, and to address the much discussed interplay between the magnetic moment and the elongation of the $\mathrm{FeAs}_{4}$ tetrahedron.

Copyright line will be provided by the publisher

[ $\left.{ }^{1}\right]$ Max-Planck-Institute for Solid State Research, Heisenbergstrasse 1, D70569 Stuttgart, Germany

\section{Foreword}

This paper is dedicated to Manuel Cardona, on the occasion of his 75th birthday. Manuel Cardona is worldfamous not only for being an outstanding and creative experimentalist, but also for having deep theoretical insights, in particular into the band structures of solids. His book [1] with Peter Yu on Fundamentals of Semiconductors, now a classic of solid-state physics, devotes a large part to the explanation of theory in simple, accessible language. For our contribution to the present Festschrift, we have chosen a topic off the main line of this special issue, but we hope that Manuel and others will appreciate our effort to follow his example and make a complicated problem more transparent.

We started working on superconductivity in the iron pnictides, and the later discovered chalchogenides, not only because we felt that this phenomenon would create the same kind of excitement as high-temperature superconductivity in cuprates had done two decades earlier, an excitement that the senior author had experienced under the leadership of Manuel Cardona, but also because we -like many other researchershad hoped that this would provide a shortcut to understanding high-temperature superconductivity. In fact, these new iron-based superconductors and the cuprates (as well as heavy-Fermion systems) share the most 
important property of having a superconducting phase close to -or even coexisting with- an antiferromagnetic phase. In the iron-based superconductors, however, the latter is metallic rather than insulating, and these superconductors may therefore lack the complications of strong-correlation Mott-Hubbard physics. In both iron-based superconductors and cuprates, phonons seem to play a minor role for the coupling, although the debate is still alive [2]. After two and a half years with enormous theoretical and experimental effort, published in over 2000 papers, [3, 4, 5] it has become clear that, although superconductivity in the iron-based superconductors could turn out to be simpler than in the cuprates, the former are in many respects more complicated than the latter -their band structures, for example, are considerably more intricate- and our present understanding is far from complete.

Trying to swim upwards through this cascade of papers has blinded at least the senior author and made him focus on finally publishing our own results. Encouraged by Manuel Cardona, to whom this could never have happened, we now take the opportunity to publish a pedagogical, self-contained account of the band structures and itinerant magnetism in the iron-based superconductors, stressing the role of symmetry and, as far as possible, reducing problems to that of diagonalizing a $2 \times 2$ matrix.

\section{Introduction}

The first report of superconductivity in an iron pnictide, specifically in F-doped LaOFeP below $5 \mathrm{~K}$ in 2006 [6, 7], was hardly noticed and only two years later, when F-doped LaOFeAs was reported to superconduct below $28 \mathrm{~K}$, the potential of iron pnictides as high-temperature superconducing materials was realized. [8] Following this discovery, more than 50 new iron superconductors with the same basic structure were discovered [9] with $T_{c}$ reaching up to $56 \mathrm{~K}$. [10] This structure is shown in Fig. 11 for the case of LaOFeAs. The common motive is a planar FeAs layer in which the Fe atoms form a square lattice, tetrahedrally coordinated with As atoms placed alternatingly above and below the hollow centers of the squares. Instead of As, the ligand could be another pnictogen $(\mathrm{P})$ or a chalcogen (X=Se or Te), but for simplicity, in this paper we shall refer to it as As. These superconductors are divided in four main families depending on their 3D crystal structure [11]: The iron chalcogenides are simple tetragonal (st) with the FeX layers stacked on top of each other (11 family). The iron pnictides have the FeAs layers separated by alkali metals (111 family), or by rare-earth oxygen/fluoride blocking layers (1111 family as in Fig.11), in st stacking, or by alkali-earth metals (122 family) in body-centered tetragonal (bct) stacking.

Iron-based superconductors share some general physical properties, although the details are often specific to families, or even to compounds. With the exception of LiFeAs, the undoped compounds are spin-density wave (SDW) metals at low temperature with the Fe spins ordered anti-ferromagnetically between nearest neighbors in the one direction and ferromagnetically in the other, thus forming stripe or double-stripe (FeTe) patterns. The values of the measured magnetic moments range from $0.4 \mu_{B} / \mathrm{Fe}$ in LaOFeAs [12], to $\sim 1 \mu_{B}$ in $\mathrm{BaFe}_{2} \mathrm{As}_{2}$ compounds, to over $2 \mu_{B}$ in doped tellurides. [3, 4, 5] At a temperature above or at the Neel temperature, which is of order $100 \mathrm{~K}$, there is a tetragonal-to-orthorhombic phase transition in which the in-plane lattice constant contracts by $0.5-1.0 \%$ in the direction of ferromagnetic order. Superconductivity sets in when the magnetic order is suppressed by pressure, electron or hole doping, or even isovalent doping on the As site, and at a much lower temperature. Both superconductivity and magnetism are found to depend crucially on the details of the crystal structure; for example is it often observed that the highest $T_{c}$ s occur in those compounds where the $\mathrm{FeAs}_{4}$ tetrahedra are regular. [13] Critical temperatures range from a few $\mathrm{K}$ in iron-phosphides to $56 \mathrm{~K}$ in SmOFeAs. The variations in the phonon spectra are, however, small and seem uncorrelated with $T_{c}$. This, together with the proximity of magnetism and superconductivity in the phase diagram, was a first indication that the superconductivity is unconventional. A stronger indication seems to come from the symmetry of the superconducting gap, which is currently a strongly debated issue. [14] Depending on the sample, and on the experimental technique, multiple gaps with $s$ symmetry and various degrees of anisotropy -but also of nodes- have been reported. [3, 4, 5] It now seems as if the gap symmetry is not universal, but material specific in these compounds. 

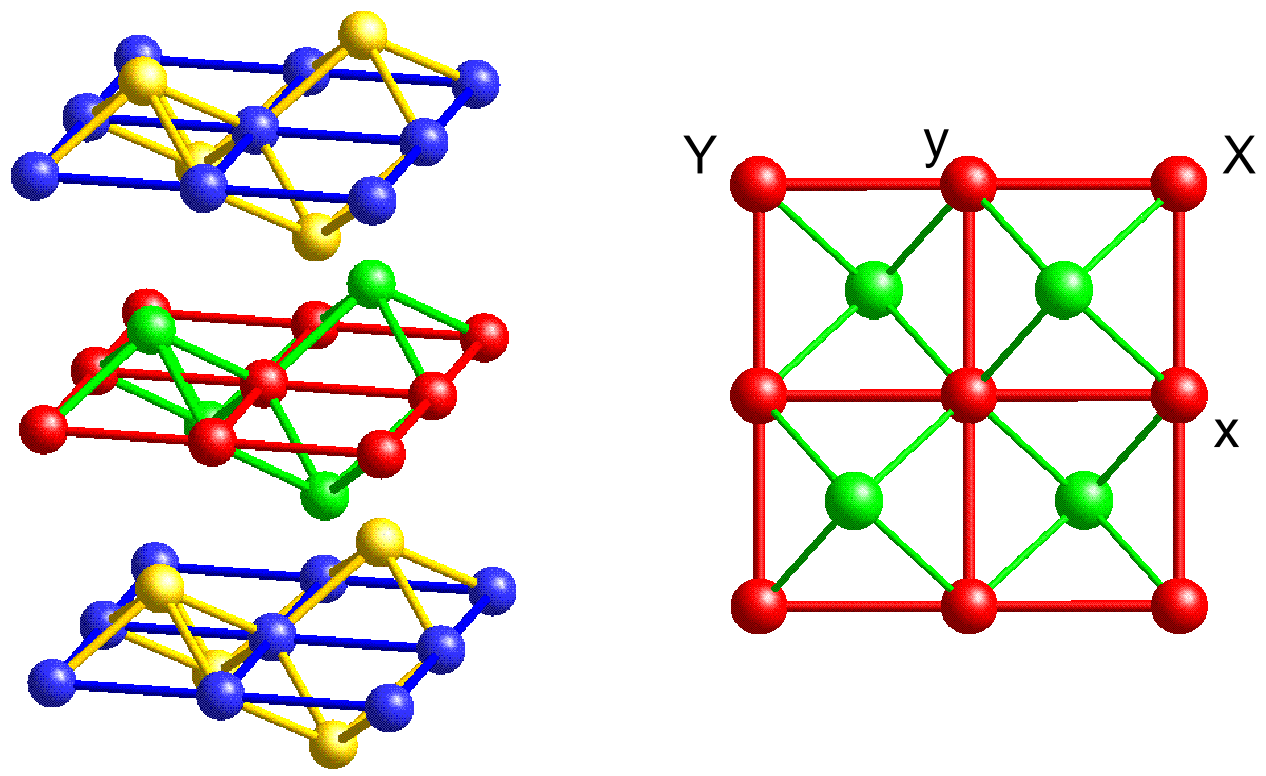

Fig. 1 The layered structure of simple tetragonal LaOFeAs. The 3D primitive cell contains one $\mathrm{Fe}_{2} \mathrm{As}_{2}$ and one $\mathrm{La}_{2} \mathrm{O}_{2}$ layer, each containing three sheets: a square planar $\mathrm{Fe}$ (red) or $\mathrm{O}$ (blue) sheet sandwiched between two planar As (green) or La (yellow) sheets. $c=874 \mathrm{pm}$. The coordination of Fe with As, or O with La, is tetrahedral. $\mathbf{x}$ and $\mathbf{y}$ are the vectors between the Fe-Fe or O-O nearest neighbors (separated by $a=285 \mathrm{pm}$ ) and $\mathbf{X}$ and $\mathbf{Y}$ are those between As-As or La-La nearest neighbors in the same sheet. The directions of those vectors we shall denote $x, y, X$, and $Y$.

Current understanding of the basic electronic structure has been reached mainly by angle-resolved photoemission (ARPES) [15, 16, 17, 18, 19, 20, 21, 22, 23, 24], quantum oscillation, and de-Haas-van-Alphen (dHvA) experiments [25, 26, 27, 28, 29, 30, 31] in combination with density-functional (DFT) calculations. [32, 33, 34, 35, 36, 37, 38, 39, 40, 41, 42, 43] All parent compounds have the electronic configuration $\mathrm{Fe}$ $d^{6}$ and are metallic. In all known cases, the Fermi surface (FS) in the paramagnetic tetragonal phase has two concentric hole pockets with dominant $d_{x z} / d_{y z}$ character and two equivalent electron pockets with respectively $d_{x z} / d_{x y}$ and $d_{y z} / d_{x y}$ character. A third hole pocket may also be present, but its character, $d_{x y}$ or $d_{3 z^{2}-1}$, as well as the sizes and shapes of all sheets, vary among different families of compounds, and, within the same family, with chemical composition and pressure. In all stoichiometric compounds, the volumes of the hole sheets compensate those of the electron sheets. The magnetically stripe-ordered phase remains metallic, but the FS becomes much smaller and takes the shape of a propeller [18] plus, possibly, tiny pockets.[25]

Given the strong tendency to magnetism, and the low value of the calculated electron-phonon interaction, [44, 45, 46] spin fluctuations are the strongest candidate for mediating the superconductivity. Alternative scenarios have been proposed, in which superconductivity is due to magnetic interactions in the strong-coupling limit, polarons, or orbital fluctuations. [47] Models for spin fluctuations are based on the weak-coupling, itinerant limit, with superconductivity related to the presence of strong nesting between hole and electron sheets of the paramagnetic Fermi surface, which is also held responsible for the instability towards magnetism. [42, 43, 48] This possibility has been investigated using more ore less sound models of the band structure, combined with different many-body methods (RPA, FLEX, frG, model ME calculations) which do seem to agree on a picture with competing instabilities towards magnetism and superconductivity. [42, 43, 49, 50, 51, 52, 53, 54, 55, 56, 57, 58, 59, 60] The superconducting phase should be characterized by multiple gaps, with $s$ and $d$ symmetries almost degenerate. Modifying the shape and 
orbital characters of the different sheets of the Fermi surface by doping, pressure, or chemistry can influence the leading instability and affect the structure of the gap. As a result, a reasonable, qualitative picture of the materials trend, such as the dependence of $T_{c}$ and gap symmetry on the tetrahedral angle, has evolved. [52, 57] Most experimental evidence seems to support this picture, but several points remain controversial. A badly understood issue is how to include 3D effects, which is particularly serious for the bct 122 compounds.

Another problem concerns the magnetism: While it is true that spin-polarized DFT (SDFT) calculations reproduce the correct atomic coordinates and stripe-order of the moment, the magnitude of the moment is, except in doped $\mathrm{FeTe}$, at least two times larger than what is measured by neutron scattering, or inferred from the gaps measured by ARPES, [30, 61], dHvA, and optics, [62] albeit much smaller than the saturation moment of $4 \mu_{B} / \mathrm{Fe}$. Suppressing the too large moments in the calculations will, however, ruin the good agreement for the structure and the phonon spectra. [45, 63, 64, 65, 66] This over-estimation of the moment is opposite to what was found 25 years ago for the superconducting cuprates where the SDFT gave no moment, but is typical for itinerant magnets close to a magnetic quantum critical point (QCP). [63] The magnetic fluctuations in time and space have been described [67] using a localized Heisenberg model with competing ferro- and antiferromagnetic interactions between respectively first and second-nearest neighbors, but to reconcile this model with the partly metallic band structure is a problem. [48, 68, 69, 70, 71] Another possible solution of the moment problem in SDFT is that moments of the predicted size are present, but fluctuate on a time scale faster than what is probed by the experiments. [63] In fact, two recent studies of realistic, DFT-derived multi-band Hubbard models solved in the dynamical mean-field approximation (DMFT) show that the magnetism has two different energy scales. [72, 73] It is therefore possible that the electronic correlations after all do play a role in these multi-band, multi-orbital materials. [74, 75]

Experiments and calculations have revealed a marked interplay between the details of the band structure and the superconducting properties. Most of these observations are empirical and we feel that there is a need to explain the origin of such details. In this paper, we therefore attempt to give a self-contained, pedagogical description of the paramagnetic and spin-polarized band structures. Specifically, we discuss the Fe $d$ As $p$ band-structure topology, causing the pseudogap at $d^{6}$ as well as numerous Dirac cones, the interlayer hopping in the simple-tetragonal and body-centered-tetragonal structures, the spin-spiral band structures, and the band-resolved magnetic energies. In all of this, the covalency between Fe $d$ and As $p$ is found to play a crucial role. Applications to superconductivity are beyond the scope of the present paper.

In Sect. 2 we explain the structure of a single, isolated FeAs layer and use the glide mirror to reduce the primitive cell to one FeAs unit and have $\mathrm{k}$ running in the large, square Brillouin zone (BZ) known from the cuprates. Halving the number of bands will prove important when it comes to understanding the multiorbital band structure. In Sect. 3 we show that this band structure may be generated and understood from downfolding, [76] of the DFT Hilbert space for LaOFeAs to a basis set consisting of the five Fe $d$, localized Wannier orbitals, or -as we prefer-including explicitely also the three As $p$ orbitals. Even the latter $8 \times 8$ tight-binding (TB) Hamiltonian, $h(\mathbf{k})$, has long-ranged $p p$ and $p d$ hoppings due to the diffuseness of the As $p$ orbitals, and its accurate, analytical matrix elements are so spacious that they will be published at a different place. [77] The crucial role of the As $p$ orbitals for the low-energy band structure, the electron bands in particular, and the presence of a $d^{6}$ pseudogap is emphasized. The different sheets of the FS are discussed. In Fig. 2 we show the factorization of the Bloch waves along the lines and points of high symmetry in the large BZ. The high symmetry of the single, tetragonal layer allows many bands to cross and leads to linear dispersions, and even to Dirac cones. Our understanding of this generic band structure of a single layer then allows us to discuss standard DFT calculations for specific materials. This is done in Sect.4] where we first see that increasing the As height moves an antibonding $p_{z} / d_{x y}$ level down towards the degenerate top of the $d_{x z} / d_{y z}$ hole bands, with which it cannot cross, and thereby causes the inner, longitudinal band to develop a linear dispersion. Interlayer hopping is shown to proceed mainly via the As $p_{z}$ orbital and to have a strength and $\left(k_{x}, k_{y}\right)$-dependence which depends on the material family. This hopping is strongest for the bct structure where the As atoms in neighboring layers face each other. In st SmOFeAs and for $k_{z}$ at the edge of the 3D BZ, the antibonding $p_{z} / d_{x y}$ level reaches the top of the hole bands and forms a Dirac cone together with the longitudinal hole band. In LiFeAs and FeTe the Dirac 


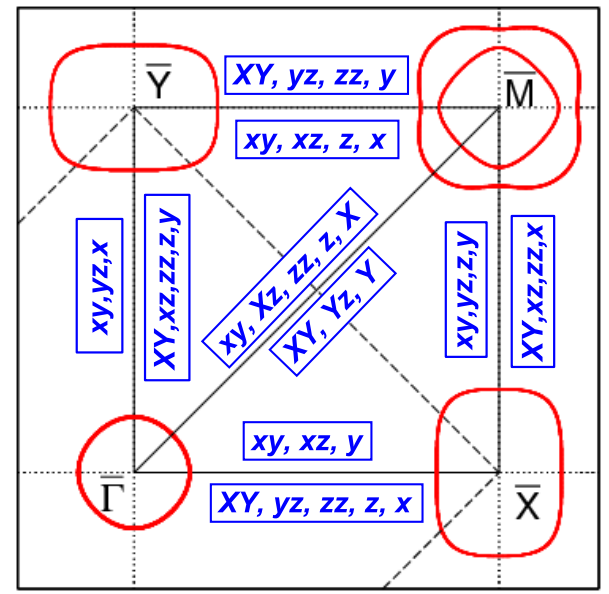

Fig. 2 Upper-right quarter of the large Brillouin zone (BZ) for the glide-mirror space group of the single FeAs layer (black), the factorization of the band Hamiltonian (blue), and the LaOFeAs Fermi surface (red). The BZ for merely the translational part of the space group has half the area and is folded-in as indicated by the dashed black lines. In order to distinguish the corners, $\mathrm{M}$, and edge midpoints, $\mathrm{X}$, of these two square BZs, we use an overbar for the large BZ. Hence $\Gamma=\bar{\Gamma}, M=\bar{X}$, and $\mathrm{X}$ is the common midpoint of the $\bar{X} \bar{Y}$ and $\bar{\Gamma} \bar{M}$-lines. The folding causes all three hole pockets to be centered at $\Gamma$ and the two electron pockets be centered at $M$ with their axes crossed. The blue boxes along the lines of high symmetry contain the orbitals whose Bloch-sums may hybridize (belong to the same irreducible representation). At the high-symmetry points, this factorization of the Hamiltonian into diagonal blocks is as follows: $\bar{\Gamma}[x y][X Y][X z, X][Y z, Y][z z, z], \overline{\mathrm{X}}[x z][x y, y][y z, z][X Y, z z, x]$, and $\overline{\mathrm{M}}[X Y][z z][x y, z][X z, X][Y z, Y]$. With often used notations [43], the inner and outer sheet of the $\overline{\mathrm{M}}$-centered $x z / y z$-like hole pockets are respectively $\alpha_{1}$ and $\alpha_{2}$ while the $\overline{\mathrm{X}}$ and $\overline{\mathrm{Y}}$-centered $x y / x z$ and $x y / y z$-like electrons sheets are respectively $\beta_{1}$ and $\beta_{2}$, and the $\bar{\Gamma}$-centered $x y$-like hole pocket is $\gamma$.

point is inside the BZ. The interlayer hopping not only causes the As $p_{z}$-like 2D bands to disperse with $k_{z}$, but also folds the bands into the conventional, small BZ, i.e. it couples $h(\mathbf{k})$ and $h(\mathbf{k}+\pi \mathbf{x}+\pi \mathbf{y})$. The formalism for interlayer hopping is given in Sect.4.2 and its increasing influence on the band structures of $\mathrm{BaFe}_{2} \mathrm{As}_{2}, \mathrm{CaFe}_{2} \mathrm{As}_{2}$, and collapsed $\mathrm{CaFe}_{2} \mathrm{As}_{2}$ is shown and explained, for the first time, we believe. In $\mathrm{CaFe}_{2} \mathrm{As}_{2}$, we find that the nearly linear dispersion of the $d_{x y} / p_{z}$-like electron band has developed into a full Dirac cone.

The effects of spin polarisation on the generic 2D band structure are discussed in Sect.5 We consider spin spirals which have a translationally invariant magnitude but a spiralling orientation which is given by q. Their band Hamiltonian possesses translational symmetry both in configurational and in spin-space, but independently of each other as long as spin-orbit coupling is neglected. The spin spiral therefore simply couples $h(\mathbf{k})$ to $h(\mathbf{k}+\mathbf{q})$, regardless of whether $\mathbf{q}$ is commensurable or not. For $h(\mathbf{k})$ we use the DFT $p d$ Hamiltonian derived in Sect.3. In order to keep the analysis transparent and amenable to generalization, we shall treat the exchange coupling using the Stoner model rather than full SDFT. This has the avantage that it decouples the band structure and self-consistency problems, so that we can study the band structure as a function of the exchange potential, $\Delta$. In Sect.5.2 we discuss the bands and FSs for the observed stripe order. As long as the moment is a linear function of $\Delta$, gapping requires matching of $d$-orbital characters as well as FS dimensions (nesting). For larger moments, and ferromagnetic order in the $x$ direction, the FS is different and shaped like a two-blade propeller in the $k_{y}$ direction. It is formed by crossing $d_{x y} / p_{z^{-}}$ $d_{y z \downarrow}$ and $d_{z z \downarrow} / d_{X Y \uparrow}$ bands, which cannot hybridize along the line through the blades and the hub. The resulting Dirac cone has been predicted before [78] and also observed. [31, 79] The interplay between $p d$ hybridization and magnetism is discussed using simple, analytical $4 \times 4$ models. In Sect.5.3 we first show the static spin-suceptibility, $m(\Delta) / \Delta$, calculated for stripe and checkerboard orders as functions of the electron doping in the rigid-band approximation. The low-moment solution -maybe fortuitously- 
resembles the behaviour of the observed moment as a function of doping and $\mathbf{q}$. We then discuss the electronic origin of the magnetic energies and first show how the magnetic energy may be interpreted as the difference between double-counting-corrected magnetic and non-magnetic band-structure energies. This directly relates the magnetism to the band structure and we specifically look at the origin of the magnetic energy. We find that the magnetic energy gain is caused by the coupling of the paramagnetic $d_{x y}$ hole and $d_{x y} / p_{z}$ electron bands, as well as by that of the $d_{x z}$ parts of the two other electron and hole bands. The Fermi-surface contributions to the magnetic energy are comparatively small. We can then explain why increasing the distance between the As and Fe sheets increases the stripe-ordered moment, and vice versa.

At the end, we compare our results with those of fully self-consistent SDFT spin-spiral calculations of moments and energies as functions of $\mathbf{q}$ and doping in the virtual-crystal approximation, for $\mathrm{LaO}_{1-\mathrm{x}} \mathrm{F}_{\mathrm{x}} \mathrm{FeAs}$ and $\mathrm{Ba}_{1-2 \mathrm{y}} \mathrm{K}_{2 \mathrm{y}} \mathrm{Fe}_{2} \mathrm{As}_{2}$.

\section{Structure}

The basic structural unit for the iron-based superconductors is a planar FeAs layer consisting of three sheets: (Fig. 11). In the high-temperature paramagnetic tetragonal phase, the iron atoms form a square sublattice $(a \equiv 1)$ with each Fe tetrahedrally coordinated by four As ligands. The latter thus form two $\sqrt{2} \times \sqrt{2}$ square lattices above and below the Fe plane at a vertical distance of approximately half the $a$-constant of the Fe sublattice. The Fe and As positions are thus described by respectively :

$$
\mathbf{t}=n_{x} \mathbf{x}+n_{y} \mathbf{y} \quad \text { and } \quad\left(n_{x}-n_{y} \pm \frac{1}{2}\right) \mathbf{x}+\left(n_{x}+n_{y}+\frac{1}{2}\right) \mathbf{y} \pm \frac{\eta}{2} \mathbf{z}=\mathbf{T}+\frac{1}{2}\left\{\begin{array}{c}
\mathbf{X}+\eta \mathbf{z} \\
\mathbf{Y}-\eta \mathbf{z}
\end{array}\right.
$$

where $\mathbf{x}$ and $\mathbf{y}$ are the orthogonal vectors between the Fe nearest neighbors and $n_{x}$ and $n_{y}$ take all integer values. $\mathbf{z}$ is perpendicular to $\mathbf{x}$ and $\mathbf{y}$, and has the same length. For perfect tetrahedra, $\eta=1$, and for LaOFeAs, $\eta=0.93$. Instead of $\eta \equiv \sqrt{2} \cot \theta / 2 \equiv 2 \sqrt{2} z_{\text {As }}$, it is customary to specify the As-Fe-As tetrahedral angle, $\theta$, or the internal parameter, $z_{\mathrm{As}}$. While $\mathbf{t}$ are the translations of the Fe sublattice, $\mathbf{T} \equiv$ $n_{X} \mathbf{X}+n_{Y} \mathbf{Y}$ are those of the As sublattice whose primitive translations are $\mathbf{X} \equiv \mathbf{y}+\mathbf{x}$ and $\mathbf{Y} \equiv \mathbf{y}-\mathbf{x}$. The latter are turned by $45^{\circ}$ with respect to $\mathbf{x}$ and $\mathbf{y}$, and $\sqrt{2}$ longer. The translation group of the FeAs layer is $\mathbf{T}$ and has two FeAs units per cell. These are, however, related by a glide mirror. Rather than using the irreducible representations of the 2D translation group, it is therefore simpler to use those of the group generated by the primitive Fe-translations, $\mathbf{x}$ and $\mathbf{y}$, combined with mirroring in the Fe-plane. These glide-mirror operations ("take a step and stand on your head") generate an Abelian group with only one FeAs unit per cell and irreducible representations, $\exp (i \mathbf{k} \cdot \mathbf{r})$, which are periodic for $\mathbf{k}$ in the reciprocal lattice, $h_{x} 2 \pi \mathbf{x}+h_{y} 2 \pi \mathbf{y}$, with $h_{x}$ and $h_{y}$ integer. The corresponding Brillouin zone (BZ) shown in Fig. 22 is a square, centered at the $\bar{\Gamma}$-point, $\mathbf{k}=\mathbf{0}$, with corners at the $\overline{\mathrm{M}}$-points, $\mathbf{k}=\pi \mathbf{y} \pm \pi \mathbf{x}$ and $-\pi \mathbf{y} \mp \pi \mathbf{x}$, i.e. at $\pm \pi \mathbf{X}$ and $\pm \pi \mathbf{Y}$, and edge-centers at the $\overline{\mathrm{X}}$ and $\overline{\mathrm{Y}}$-points, $\mathbf{k}= \pm \pi \mathbf{x}$ and $\pm \pi \mathbf{y}$. In this paper, we shall use this more heavy notation instead of e.g. $(\pi, \pi)$ for $\overline{\mathrm{M}}$ and $(\pi, 0)$ for $\overline{\mathrm{X}}$ as done for cuprates, because for the iron superconductors, no consensus exists about whether to use the $(x, y)$ or the $(X, Y)$ coordinate system. The overbar is used to designate the high-symmetry points in the $2 \mathrm{D}$ reciprocal space for the glide-mirror group. In conclusion, use of the glide-mirror group reduces the number of bands by a factor of two, and this is important when attempting to understand the intricacies of the band structure.

In Fig. 3 we sketch the antibonding Bloch sums of the Fe $d_{x y}$ (top) and $d_{x z}$ (bottom) orbitals, and realize that with the glide-mirror notation the former has $\mathbf{k}=\mathbf{0}$ and the latter $\mathbf{k} \cdot \mathbf{x}=\pi$. Accordingly, the top of the pure Fe $d_{x y}$ band is at $\bar{\Gamma}$, while the degenerate top of the pure $d_{x z}$ and $d_{y z}$ bands is at $\bar{M}$. We shall often return to this. (Authors who unfold without reference to the glide-mirror group, may have $\bar{\Gamma}$ and $\bar{M}$ interchanged, with the result that the $x y$ hole pocket and the two $x z / y z$ hole pockets are respectively at $\overline{\mathrm{M}}$ and $\bar{\Gamma}$. In order to avoid this confusion, it is useful to remember that the two $x z / y z$ hole pockets are those towards which the electron superellipses at $\bar{X}$ and $\bar{Y}$ are pointing).

The real 3D crystals consist of FeAs layers stacked in the $z$-direction with other layers intercalated, although the iron chalcogenides, FeX, have no intercalation. Fig 1 specifically shows LaOFeAs, for which all 


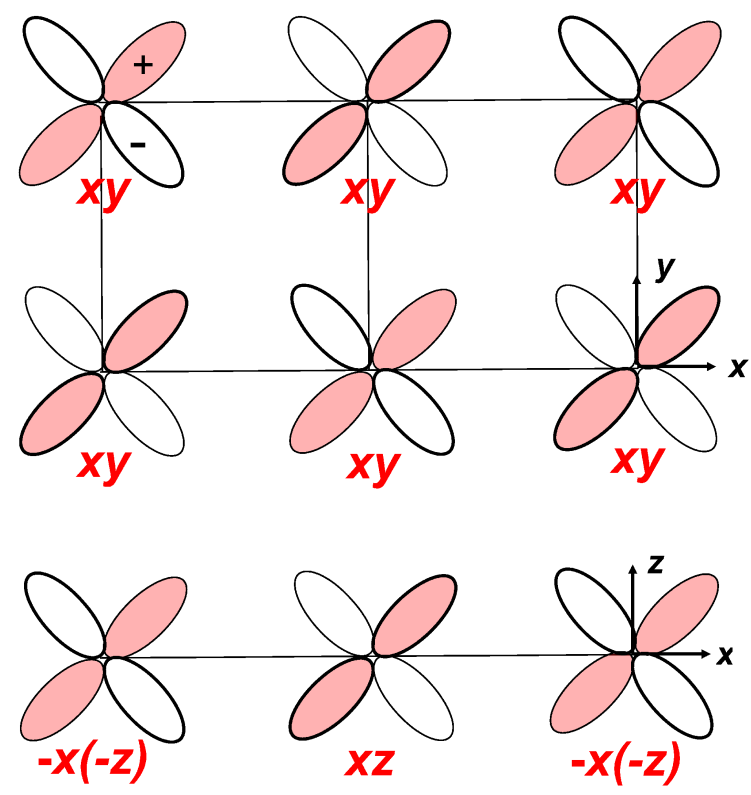

Fig. 3 Sketch of the antibonding Bloch sum of Fe $d_{x y}$ orbitals in the $x y$-plane (top) and of the antibonding Bloch sum of Fe $d_{x z}$ orbitals in the $x z$-plane (bottom). A Bloch sum is formed by adding the glide-mirrored orbital multiplied by $\exp (i \mathbf{k} \cdot \mathbf{t})$, where the glide, $\mathbf{t}$, is a primitive translation, $\mathbf{x}$ or $\mathbf{y}$, and the mirror is the Fe plane. The antibonding Bloch sum of $d_{x y}$ orbitals has $\mathbf{k}=\mathbf{0}$ and that of $d_{x z}$ orbitals has $\mathbf{k} \cdot \mathbf{x}=\pi$. That the lobes of the real Wannier orbitals avoid the As sites (Fig. 6) is indicated by enhancing the countours of the lobes pointing towards the reader.

our Wannier-orbital (3D) calculations were done, unless otherwise stated. The interlayer coupling is weak but not negligible, and it depends on the material. Although the 2D glide-mirror may take the 3D crystal into itself, as is the case for LnOFeAs, FeX, and LiFeAs, we do want to use $k_{z}$ to enumerate the states in the third direction. For the 3D crystals we shall therefore use the standard 3D translation group according to which only the $\mathbf{X}$ and $\mathbf{Y}$ translations, combined with an out-of plane translation, leave the crystal invariant. The corresponding $2 \mathrm{D}$ reciprocal lattice is $h_{X} \pi \mathbf{X}+h_{Y} \pi \mathbf{Y}=\left(h_{X}+h_{Y}\right) \pi \mathbf{y}+\left(h_{X}-h_{Y}\right) \pi \mathbf{x}$. Hence, the 3D Brillouin zone is as shown by the dashed lines in Fig. 2 (for $k_{z}=\pi / 2 c$ ), with $\overline{\mathrm{M}}$ falling onto $\bar{\Gamma}$ and with corners at $\overline{\mathrm{X}}$ and $\overline{\mathrm{Y}}$, now named $\mathrm{M}$. Interlayer hopping may thus couple the glide-mirror states at $\mathbf{k}$ with those at $\mathbf{k}+\pi \mathbf{x}+\pi \mathbf{y}$. This material-dependent coupling will be considered in Section 4 after we have explained the generic electronic structure of a single FeAs layer.

Spin-orbit interaction also invalidates the glide-mirror symmetry, but the splitting of states degenerate at $\mathbf{k}$ and $\mathbf{k}+\pi \mathbf{x}+\pi \mathbf{y}$ is at most $\frac{3}{2} \zeta_{\mathrm{Fe} 3 d} \approx 0.1 \mathrm{eV}$, and this only occurs if all three $x y, y z$, and $x z$ states happen to be degenerate and purely Fe $d$-like.

\section{Paramagnetic 2D band structure}

In this section we shall describe the generic $2 \mathrm{D}$ band structure of an isolated FeAs layer. We start by observing that the bands are grouped into full and empty, separated by a pseudogap. We then discuss the grouping of the bands into Fe $3 d$ and As $4 p$, and derive two sets of Wannier orbitals from DFT, one set describing merely the five Fe $d$-like bands and another set describing the eight Fe $d$ - and As $p$-like bands. Armed with those sets, we can return to a detailed description of the low-energy bandstructure, i.e. the one which forms the pseudogap at $d^{6}$ and the Fermi surface. This is done in subsection 3.3 where we shall see that the hybridization between -or covalency of- the As $p$ and the Fe $d$ orbitals is crucial for the band 


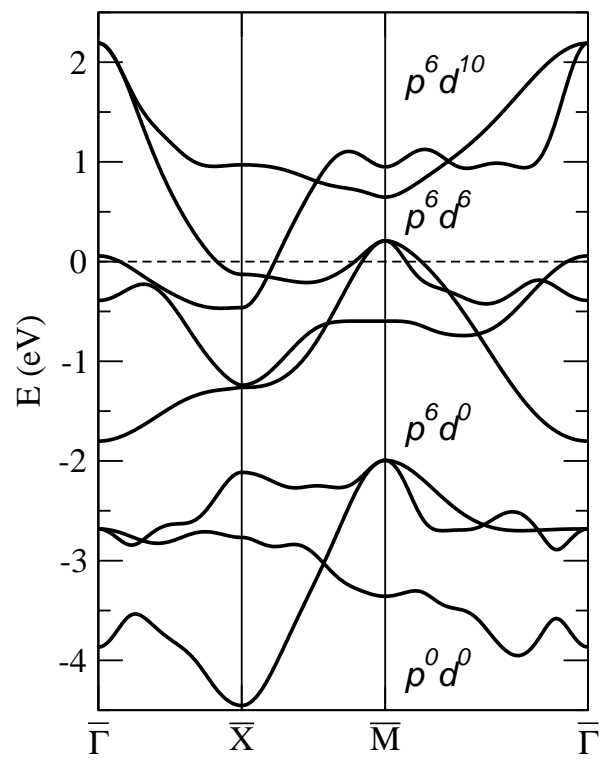

Fig. 4 Band structure of paramagnetic, tetragonal pure LaOFeAs with the experimental structure near the Fermi level $(\equiv 0)$ and for $\mathbf{k}$ along the high-symmetry lines in the large 2D Brillouin zone (Fig. 2). Band energies are in eV. These DFT-GGA bands were calculated with the NMTO method and a basis of Fe $d$ and As $p$ downfolded NMTOs. Transformation to real space yields the eight Wannier functions shown in Fig 6 The 2D bands were obtained by neglecting the interlayer hoppings and forming glide-mirror Bloch sums of the Wannier orbitals on a single FeAs layer, i.e. by appropriately flipping the signs of the intra-layer hopping integrals. The large gaps in the figure are labelled by an electronic configuration which corresponds to a $p$-set and a $d$-set of Wannier orbitals which span respectively the three lowest and the five highest bands. This $d$-set is illustrated in Fig 5 Upon electron doping in the rigid band approximation, the $\bar{\Gamma}$-centered hole pocket fills once the doping exceeds $0.1 \mathrm{e} / \mathrm{Fe}$, and when it exceeds $0.3 \mathrm{e} / \mathrm{Fe}$, also the $\bar{M}$-centered hole pockets fill.

topology. Bringing this out clearly, was in fact our original reason for deriving the eight-orbital $p d$ set, although the five-orbital $d$ set suffices to describe the low-energy band structure.

For FeTe and $\mathrm{LaOFe} A$ s the formal ionic states are respectively $\mathrm{Fe}^{2+} \mathrm{Te}^{2-}$ and $\mathrm{La}^{3+} \mathrm{O}^{2-} \mathrm{Fe}^{2+} \mathrm{As}^{3-}$. In fact, for all parents of the iron-based superconductors, the nominal electronic configuration is ligand $p^{6}$ Fe $d^{6}$. The generic 2D band structure is shown in Fig. 4 for energies ranging from $4.5 \mathrm{eV}$ below to 2.5 $\mathrm{eV}$ above the Fermi level and along the high-symmetry lines of the BZ (Fig. 2). In the energy range considered, there are eight bands which are seen to separate into three low-energy and five high-energy bands. They may be called respectively the ligand $p$ - and iron $d$-bands, and the corresponding electron count is as written on the figure. At $p^{6} d^{6}$ the two uppermost bands are seen to be detached from the rest, except at one (Dirac) point along the $\overline{\mathrm{X}} \overline{\mathrm{M}}$-line where two bands cross, because their Bloch functions are respectively even and odd with respect to reflection in a vertical mirror parallel to $\overline{\mathrm{X}} \overline{\mathrm{M}}$ and containing nearest-neighbor As atoms. If the energy of this crossing could be moved up, above the relative band maxima at $\bar{\Gamma}$ and $\overline{\mathrm{M}}$, it would drag the Fermi level along and the material would transform into a zero-gap semiconductor. For the iron-based superconductors, however, the Fermi level is merely in a pseudogap and the Fermi surface (FS) consists of a $\bar{\Gamma}$-centered hole pocket, two $\bar{M}$-centered hole pockets, and two compensating electron pockets centered at respectively $\overline{\mathrm{X}}$ and $\overline{\mathrm{Y}}$ (Fig. 22).

\subsection{Fe $d$ five-orbital Wannier basis}

Characterizing the five upper bands as Fe $d$ is sound, because they can be spanned exactly by five Wannier functions [43] which behave like Fe $d$-orbitals. This can be seen in Fig. 5. Our Wannier functions were constructed [76] to have $d$ character on the central Fe site and no $d$ character on any other Fe site. This 


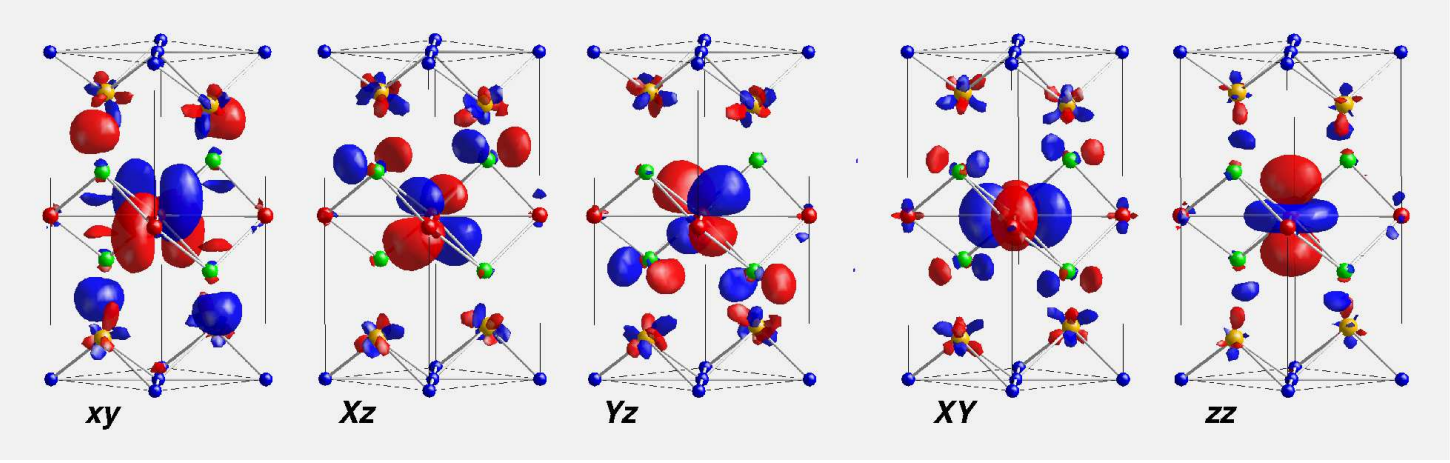

Fig. 5 The set of five Fe $d$-like Wannier orbitals (downfolded and orthonormalized NMTOs) which span the five $\mathrm{LaOFe} A s$ bands extending from $-1.8 \mathrm{eV}$ below to $2.2 \mathrm{eV}$ above the Fermi level. Shown are the positive and negative contours, $\chi_{m}(\mathbf{r})= \pm|c|$, with the former in red and the latter in blue. Orientation and coloring (Fe red, As green, La yellow, and O blue) as in Fig.1 The three orbitals to the left and the two to the right would belong to respectively the $t_{2}$ and $e$ representations, had the point symmetry been tetragonal. Now, $t_{2}$ split into $a\left(d_{x y}\right)$ and $t\left(d_{x z}, d_{y z}\right)$. Note that the $t$ orbitals $d_{x z} \equiv\left(d_{X z}-d_{Y z}\right) / \sqrt{2}$ and $d_{y z} \equiv\left(d_{X z}+d_{Y z}\right) / \sqrt{2}$, whose Bloch sums form the proper linear combinations for $\mathbf{k}$ along $\bar{\Gamma} \bar{X}$ and $\bar{X} \bar{M}$ (Fig.2), are not simply $45^{\circ}$-turned versions of $d_{X z}$ and $d_{Y z}$ shown here, in particular because the $p$ tails of the latter are on different pairs of arsenics. The $p$ tails are thus always directed towards the nearest As neighbors in the same plane, i.e. they are $X$ or $Y$, and they antibond with the $t$ head.

makes them localized Wannier orbitals. The five bands of course have characters other than Fe $d$, and those characters are mixed into the Fe $d$ Wannier orbitals. This by-mixing follows the point symmetry in the crystal. Specifically, the Fe $d_{x y}$ Wannier orbital has on-site Fe $p_{z}$ character breaking the horizontal-mirror symmetry of the pure $d_{x y}$ orbital, as well as strong off-site $p_{z}$ character on all four As neighbors. The sign of the As $p_{z}$ character is antibonding to Fe $d_{x y}$ because the As $p$ hybridization pushes the Fe $d$ band up in energy. The corresponding nodes between the $\mathrm{Fe} d$ and As $p$ tails make neighboring lobes difficult to see in the figure. Hence, only the As $p_{z}$ lobes pointing towards the La layers are big. Similarly, the Fe $d_{X z}$ Wannier orbital antibonds with $p_{X}$ on the two As neighbors in the $X$ direction, and $\mathrm{Fe} d_{Y z}$ antibonds with $p_{Y}$ on the two As neighbors in the $Y$ direction. If the Fe-site symmetry had been exactly tetragonal, the three above-mentioned Wannier orbitals would have been degenerate and transformed according to the $t_{2}$ irreducible representation. However, the non-tetrahedral environment, e.g. flattening of the tetrahedron $(\eta<1)$, increases the energy of the $d_{x y}$ orbital above that of the $d$ orbitals belonging to $t$, i.e. $d_{X z}$ and $d_{Y z}$ or, equivalently, $d_{x z}$ and $d_{y z}$. In LaOFeAs, the energy of $d_{x y}$ is $\sim 0.1 \mathrm{eV}$ above that of $d_{t}$. The two remaining Wannier orbitals, $d_{3 z^{2}-1} \equiv d_{z z}$ and $d_{y^{2}-x^{2}} \equiv d_{X Y}$, antibond less with As $p$ because their lobes point between the arsenics. Fe $d_{z z}$ is seen to antibond with $p_{z}$ on the four As neighbors and $\mathrm{Fe} d_{X Y}$ antibonds with $p_{Y}$ on the two As neighbors in the $X$ direction, and with $p_{X}$ on the two As neighbors in the $Y$ direction. In tetrahedal symmetry these two orbitals would transform according to the $e$ representation, and that holds quite well also in the real materials where the orbitals are degenerate within a few meV. Their energy is $\sim 0.2 \mathrm{eV}$ below that of the $d_{X z}$ and $d_{Y z}$ orbitals. This $e-t_{2}$ splitting of a central $d$ shell in a tetrahedron having $p$ orbitals at its corners is an order of magnitude smaller than the $t_{2 g}-e_{g}$ splitting in an octahedron which allows for better alignment of the $p$ and $d$ orbitals. The $\sim 0.2 \mathrm{eV} e-t_{2}$ splitting in $\mathrm{LaOFeAs}$ is 20 times smaller than the width of the Fe $d$-band structure in Fig. 4 and does not cause separation into two lower $e$ and three higher $t_{2}$ bands with a pseudogap at $d^{4}$. Nevertheless, the $t_{2}$ and $e$ orbitals do play quite different roles in forming the band structure near the Fermi level, as we shall see later.

Whereas in cubic perovskites, including the cuprate superconductors, the effective $d d$ hopping in the separated $t_{2 g}$ and $e_{g}$ bands proceeds almost exclusively through the $p$ tails, which are placed between the nearest-neighbor $d$ orbitals, the effective $d d$ hopping in the iron-based superconductors proceeds directly 


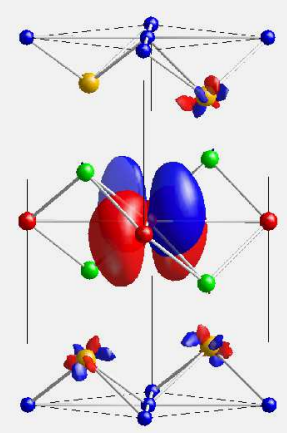

$x y$
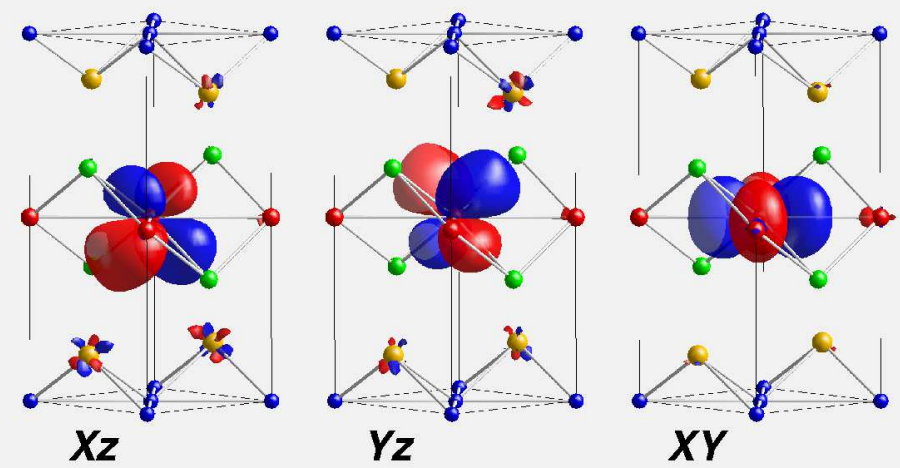

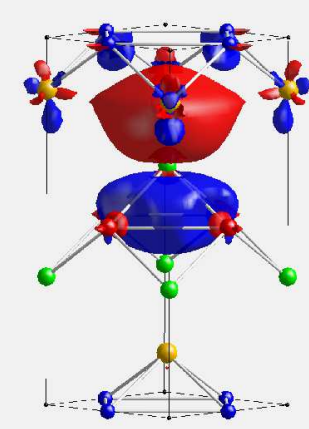

$\boldsymbol{z}$

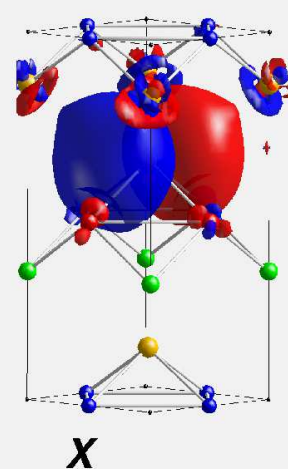

$\boldsymbol{X}$
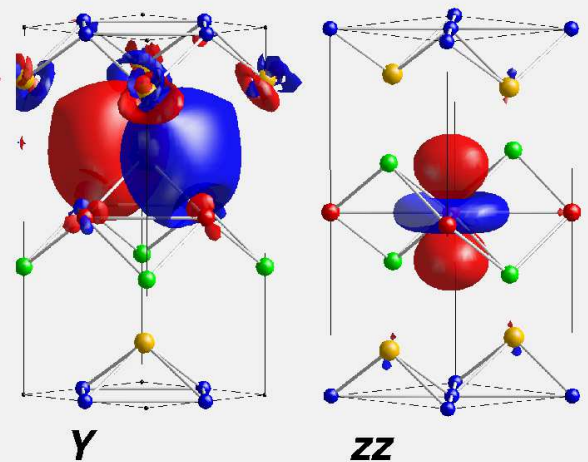

$\mathbf{Z Z}$

Fig. 6 Same as Fig.5 but for the set of eight As $p$ - and Fe $d$-like Wannier functions which span the entire band structure shown in Fig. 4

between nearest-neighbor $d$ orbitals on the square lattice as well as via the $p$ tails lying above and below the plane of the $d$ orbitals.

Tabulations of the hopping integrals for the $d$-orbital Hamiltonian may be found in Refs. [43] and [59].

\subsection{Fe $d$ As $p$ eight-orbital Wannier basis and its Hamiltonian}

In order to explain the band structure, and in particular the interlayer coupling in Sect. 4, we find it useful to exhibit the As $p$ characters explicitly. We therefore choose not to downfold the As $p$ channels, but span the entire eight-band structure in Fig. 4 by the eight As $p$ and Fe $d$ Wannier orbitals; all other channels remain downfolded [76]. These eight orbitals are shown in Fig.6 Due to the lack of As $p$ tails, the Fe $d$ orbitals of this $p d$ set are more localized than those of the $d$ set and the integrals for hopping between them have a shorter range. This basis set is also more suited for including the on-site Coulomb correlations. The As $p$ orbitals are, on the other hand, quite diffuse and give rise to strong and long-ranged $p p$ and $p d$ hoppings inside the layer. For the $p_{z}$ orbital, this is partly due to its La $d$ and $\mathrm{O} p$ tails. This situation is very different from the one found in the cuprates, where long-ranged $p p$ hopping is blocked by the presence of $\mathrm{Cu}$ in the same plane. Although the orbitals of the AsFe $p d$ set resemble atomic orbitals more than those of the $d$ set, they do tend to avoid the space covered by the other orbitals in the set: The Fe $d$ orbitals avoid the As sites and the As $p$ orbitals avoid the Fe sites. This distorts in particular the Fe $t_{2}$ orbitals. The on-site energies, $\epsilon_{\alpha}$, and the nearest-neighbor hopping integrals, $t_{\alpha, \beta}^{n_{x}, n_{y}}$, are given in the table below. Here, all energies are in $\mathrm{eV}$ and the hopping integral is the matrix element of the Hamiltonian between Wannier orbitals $\alpha$ and $\beta$ with $n_{x} \mathbf{x}+n_{y} \mathbf{y}$ being the vector from $\alpha$ to $\beta$, projected onto the Fe plane. All hopping integrals needed to obtain converged energy bands together with their analytical expressions will be published in Ref. [77]. 
For LaOFeAs, $a=285 \mathrm{pm}$ (and, within a few per cent, the same for the other iron-based superconductors). The energies of the $p$ and $d$ orbitals are respectively -1.8 and $-0.7 \mathrm{eV}$. This $1.1 \mathrm{eV} p d$ separation is merely a fraction of the $7 \mathrm{eV} p d$-band width and it therefore seems fair to claim that the band structure is more covalent than ionic. Nevertheless, it does split into three lower As $p$-like and five upper Fe $d$-like bands as noted above. The band structure fattened by the weight of each of the eight Wannier orbitals of the $p d$ set is shown in Fig.7 Here and in the following we write $x y$ for $\mathrm{Fe} d_{x y}, t$ for $\mathrm{Fe} d_{t}, z$ for As $p_{z}$, a.s.o.. The strange wiggles of some of the bands may be seen to have strong $z$ character and this tells us that the reason for those wiggles is intra-layer hopping via the LaO layers, whose orbitals are downfolded mainly into the As $z$ orbital.

\begin{tabular}{|cccccc|}
\hline$\epsilon_{x y}=-0.72$ & $\epsilon_{x z}=-0.65$ & $\epsilon_{X Y}=-0.75$ & $\epsilon_{z z}=-0.62$ & $\epsilon_{z}=-1.75$ & $\epsilon_{x}=-1.93$ \\
$t_{x y, x y}^{1,0}=0.23$ & $t_{x z, x z}^{1,0}=0.23$ & $\begin{array}{c}t_{X Y, X Y}^{1,0}=-0.31 \\
t_{X Y, z z}^{1,0}=-0.15\end{array}$ & $t_{z z, z z}^{1,0}=-0.06$ & $t_{z, z}^{1,0}=0.32$ & $t_{X, X}^{1,0}=0.24$ \\
& & $t_{z, X}^{1,0}=-0.27$ & $t_{X, X}^{11}=0.53$ \\
$t_{x y, X}^{\frac{1}{2}, \frac{1}{2}}=0.22$ & $t_{X z, X}^{\frac{1}{2}, \frac{1}{2}}=0.72$ & $t_{X Y, X}^{\frac{1}{2},-\frac{1}{2}=0.62}$ & $t_{z z, z}^{\frac{1}{2}, \frac{1}{2}}=-0.41$ & $t_{x y, z}^{\frac{1}{2}, \frac{1}{2}}=0.52$ & $t_{z z, X}^{\frac{1}{2}, \frac{1}{2}}=0.31$ \\
& $t_{Y z, Y}^{\frac{1}{2}} \frac{1}{2}=-0.49$ & & & & \\
\hline
\end{tabular}

\subsection{D Bands and Fermi surface}

We now follow the bands around the $d^{6}$ pseudogap and begin with the Fermi surface near $\bar{\Gamma}$.

The $\bar{\Gamma}$-centered hole pocket is seen to have $x y$ character and, as sketched at the top of Fig. 3 , its Bloch function $d d$-antibonds with all four nearest $\mathrm{Fe}$ neighbors. If in this figure we imagine inserting the $x y$ orbital of the $d$ set (Fig. 5), we realize that the sum of the As $p_{z}$ tails cancel. In fact, none of the other 8 orbitals in the $p d$ set can mix with the Bloch sum of $x y$ orbitals at $\bar{\Gamma}$. This we have stated in the caption to Fig.2 2 together with the selection rules for all other high-symmetry points. The selection rules for the high-symmetry lines are given in blue on the figure. For $\mathbf{k}$ moving from $\bar{\Gamma}$ towards $\overline{\mathrm{X}}$, the $x y$ band is seen to disperse downwards because in the $x$-direction, the character of the wavefunction goes from $d d$ antibonding to bonding and, at the same time, the band gets repelled by the above-lying $x z / y$ band whose $x z$ orbitals point in the direction of the $\mathbf{k}$-vector, i.e. the longitudinal $t$ band. The corresponding inter-band matrix element increases linearly with the distance from $\bar{\Gamma}$, whereby the downwards curvature of the $x y$ band is enhanced by about $10 \%$. The resulting hole band mass is about twice that of a free-electron.

At $\overline{\mathrm{X}}$, the $x y$ Bloch sum bonds between nearest Fe neighbors in the $x$ direction and antibonds between those in the $y$ direction. In addition, weak hybridization with a $2 \mathrm{eV}$ lower-lying As $y$ band provides $y$ character to antibond between the $x y$ orbitals in the $y$ direction. This pushes the $x y$ band up at $\overline{\mathrm{X}}$ by 0.2 $\mathrm{eV}$ to $-0.4 \mathrm{eV}$. As $\mathbf{k}$ now moves on from $\overline{\mathrm{X}}$ towards $\overline{\mathrm{M}}$, the Bloch sum of $x y$ orbitals becomes bonding between Fe nearest neighbors in the $y$ direction as well, whereby the As $z$ tails (Fig [5) no longer cancel and their antibonding contribution increases linearly with $k_{y}$. This causes the band to disperse strongly upwards, to a maximum $1 \mathrm{eV}$ above the Fermi level. As can be seen from Fig. 7 this strong change of band character may also be explained as the result of strong $p d$ hybridization and avoided crossing of a pure $x y$ band dispersing downwards from $\overline{\mathrm{X}}$ to $\overline{\mathrm{M}}$ and a pure $z$ band dispersing strongly upwards due to long-range hopping, partly via La and $\mathrm{O}$ (see Fig. 6). Corresponding to the $1.3 \mathrm{eV}$ upwards dispersion of the $x y / z$ antibonding band along $\overline{\mathrm{X}} \overline{\mathrm{M}}$, we see a downwards dispersion the $z / x y$ bonding band. At $\overline{\mathrm{M}}, x y$ and $z$ hybridize, but only with each other: the pure $x y$ level is at $-2.0 \mathrm{eV}$, the pure $z$ level at $-0.4 \mathrm{eV}$, and the $x y-z$ hybridization is $2 \mathrm{eV}$, thus pushing the antibonding, predominantly $z$-like level up to $+0.9 \mathrm{eV}$ and the bonding, predominantly $x y$-like level down to $-3.4 \mathrm{eV}$.

The $t$-bands which form the $\bar{M}$-centered hole pockets exhibit a very similar behavior as the $x y$ band when, instead of going along the path $\bar{\Gamma}-\overline{\mathrm{X}}-\overline{\mathrm{M}}$ (or $\bar{\Gamma}-\overline{\mathrm{Y}}-\overline{\mathrm{M}}$ ), we go along the path $\overline{\mathrm{M}}-\overline{\mathrm{X}}-\bar{\Gamma}$ for the $x z$ band and along $\bar{M}-\bar{Y}-\bar{\Gamma}$ for the $y z$ band. The avoided $p d$ crossing is now between a pure $x z$ band dispersing downwards from $\overline{\mathrm{X}}$ to $\bar{\Gamma}$ and a pure $y$ band dispersing strongly upwards; these dispersions are strong because $\mathbf{k}$ changes in the direction of strong hoppings, $d d \pi$ and $p p \pi$, respectively. At $\overline{\mathrm{X}}$, the $x z$ band is 

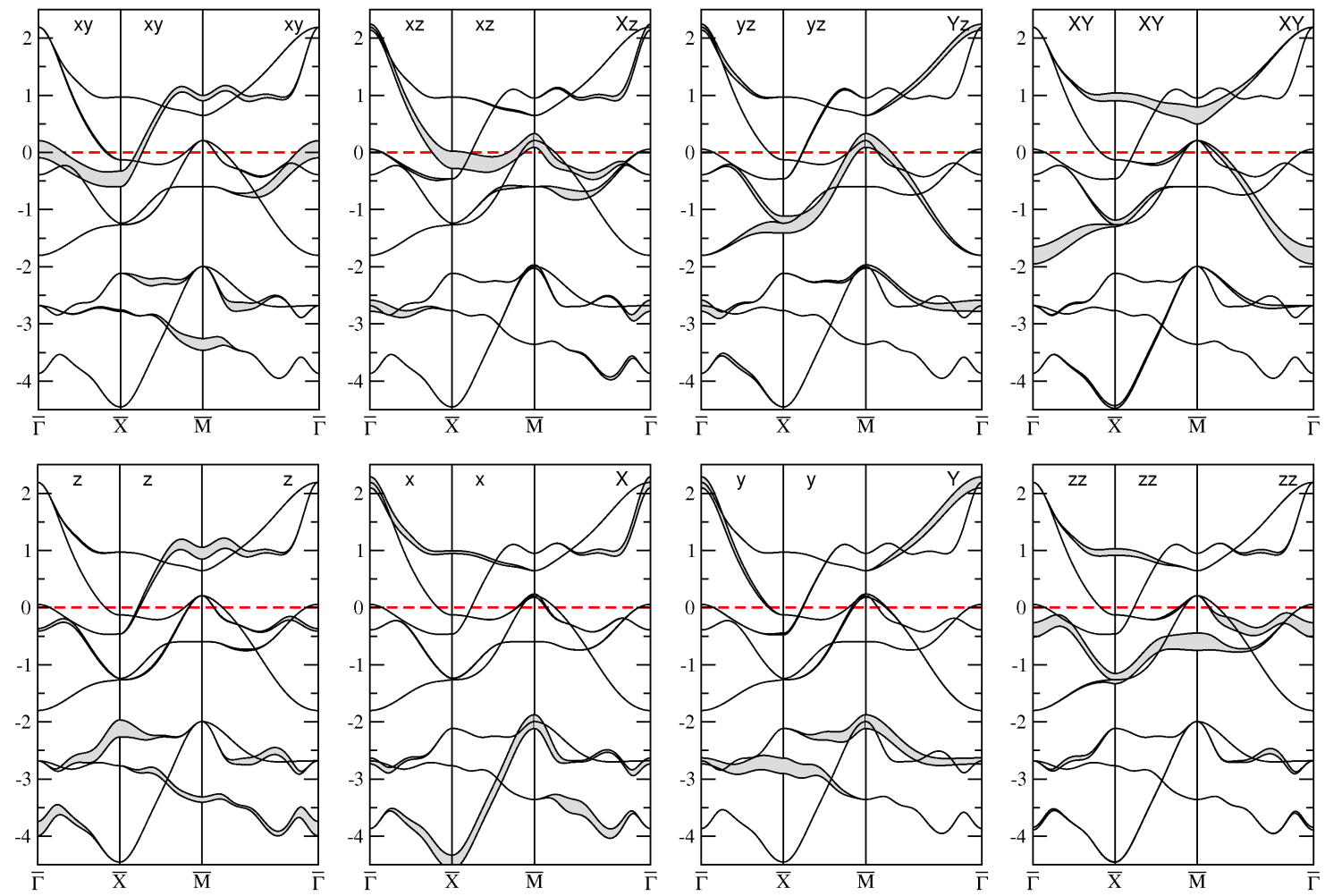

Fig. 7 Band structure from Fig. 4f fattened by the character of each of the eight Wannier orbitals in the $p d$ set (Fig. 6). A fatness was obtained by perturbing the on-site energy of the orbital in question. That two bands share the same fatness means that they hybridize. In order to concentrate the fatness onto as few bands as possible we chose the appropriate linear-combination of $t$ orbitals (see Fig. 2) for each high-symmetry line. The $\bar{\Gamma} \bar{M}$-line was chosen as the one in the $X$ direction.

pure and merely $0.1 \mathrm{eV}$ below the Fermi level. At $\bar{\Gamma}$, the pure $x z$ level is at $-1 \mathrm{eV}$, the pure $y$ level is at $+0.5 \mathrm{eV}$, and the hybridization between them is over $2 \mathrm{eV}$ thus pushing the antibonding, predominantly $y$-like level to $+2.2 \mathrm{eV}$ above the Fermi level and the bonding, predominanly $x z$-like level down to -2.7 $\mathrm{eV}$. Whereas along $\bar{\Gamma} \overline{\mathrm{X}}$, the $x y$ and $x z / y$ bands hybridize and therefore cannot cross, along $\overline{\mathrm{X}} \overline{\mathrm{M}}$, they $d o$ cross because the Bloch sums with $k_{x}=\pi$ of $\mathrm{Fe} x y$ and Fe $x z$ orbitals are respectively even and odd upon reflection in the As-containing vertical mirror perpendicular to the $x$ direction. In other words, they belong to different irreducible representations (Fig. 2). Since outside the $\overline{\mathrm{X}} \overline{\mathrm{M}}$-line, hybridization between them is no longer forbidden, the accidental degeneracy is at a Dirac point in 2D (a line in 3D). To separate the two bands would require moving the $x y / y$ above the $x z$ level at $\overline{\mathrm{X}}$.

Near $\overline{\mathrm{X}}$, the $x y$ - and $x z$-like bands are close in energy and both have minima. The minimum of the $x y$-like band curves steeply upwards towards $\bar{M}$ due to strong hybridization with $z$, and that of the $x z$-like band curves steeply upwards towards $\bar{\Gamma}$, due to strong hybridization with $y$, but is flat towards $\bar{M}$, which is the direction transversal to the dominating $d d \pi$ hopping. These two bands hybridize weakly with each other, except on the $\overline{\mathrm{X}} \overline{\mathrm{M}}$-line. As a result, they form a lower and an upper band of which the latter cuts the Fermi level at an $\overline{\mathrm{X}}$-centered electron pocket. As long as the Fermi level is well above the band crossing along $\overline{\mathrm{X}} \overline{\mathrm{M}}$, the shape is that of a 4th-order superellipse. This ellipse points towards $\overline{\mathrm{M}}$ where its character is mainly $x y / z$ and is flat towards $\bar{\Gamma}$, where its character is predominantly $x z / y$. 
The two bands forming the $\overline{\mathrm{X}}$-centered electron pocket can be modeled by the Hamiltonian,

$$
H\left(k_{x}, k_{y}\right)=\left(\begin{array}{cc}
\epsilon_{x y} & 0 \\
0 & \epsilon_{x z}
\end{array}\right)+\left(\begin{array}{cc}
\frac{\tau k_{x}^{2}}{m_{x y, x y}^{x}}+v_{x y, x y}^{y} k_{y} & v_{x y, x z}^{x} k_{x} \\
v_{x y, x z}^{x} k_{x} & \frac{\tau k_{x}^{2}}{m_{d d \pi}}+\frac{\tau k_{y}^{2}}{m_{d d \delta}}
\end{array}\right),
$$

in the basis of two effective (downfolded) Bloch orbitals, $x y$ and $x z$, and where the origin of $\left(k_{x}, k_{y}\right)$ is taken at $\overline{\mathrm{X}}$. Note that the dispersion of the effective $x y$ band towards $\overline{\mathrm{M}}$ is nearly linear for energies not too close to $\epsilon_{x y}$ due to the avoided crossing of the pure $x y$ and $z$-bands and the linear increase of their hybridization (see Fig.77, we shall return to this). In expression (2), $\epsilon_{x y}=-0.44 \mathrm{eV}$ and $\epsilon_{x z}=-0.13 \mathrm{eV}$ are the levels at $\overline{\mathrm{X}}$ with respect to the Fermi level, $v_{x y, x y}^{y}=0.53$ and $v_{x y, x z}^{x}=-0.29 \mathrm{eV} \cdot a$ are band slopes, i.e. group velocities, and $m_{x y, x y}^{x}=2.9, m_{d d \pi}=2.3$, and $m_{d d \delta}=-7$ are the band masses relative to that of a free electron. Negative masses are those of holes. Moreover, $\tau \equiv\left(a_{0} / a\right)^{2} \mathrm{Ry}=0.47 \mathrm{eV}$ with $a_{0}$ the Bohr radius. The numerical values are for pure $\mathrm{LaOFeAs}$ with the experimental structure and were obtained by fitting the result of an LAPW calculation near the Fermi level. Like everywhere else in this paper, $k$ is in units of the inverse Fe-Fe nearest-neighbor distance, $1 / a=1 / 285 \mathrm{pm}$. By symmetry, the $x y$ - and $y z$-like bands give rise to a $\bar{Y}$-centered electron pocket which also points towards $\bar{M}$, where the character is mainly $x y / z$, and is flat towards $\bar{\Gamma}$ with predominant $y z / x$ character. This is illustrated in Fig. 8 by projection of the Fermi surface onto the various orbitals.

Although none of the sheets of the paramagnetic Fermi-surface have major $e$ character, the two $e$ orbitals play a decisive role in the formation of the $d^{6}$ pseudogap. The pure $z z$ band is centered at $-0.6 \mathrm{eV}$ and disperses so little that it lies entirely below the Fermi level. At $\overline{\mathrm{M}}$, the $z z$ band is pure, and at $\bar{\Gamma}$, hybridization with the $z$ band pushes it up by $0.6 \mathrm{eV}$, an amount larger than the width of the pure $z z$ band, to $-0.4 \mathrm{eV}$. The pure $X Y$ band, on the other hand, is broad because the lobes of the nearest-neighbor $X Y$ orbitals point directly towards each other. This band has its minimum at $\bar{\Gamma}$, saddlepoint at $\overline{\mathrm{X}}$, and maximum at $\bar{M}$. Both extrema, at respectively $-1.8 \mathrm{eV}$ and $+0.6 \mathrm{eV}$, are pure. At intermediate energies, the $X Y$ band is however gapped in large regions centered at $\bar{X}$ and $\bar{Y}$ by avoided crossings with the $z z$ band.

Near the $\bar{M} \bar{\Gamma}$-lines, where the $X Y$ and $z z$ bands cannot hybridize, the $X Y$ band has an avoided crossing near $\bar{M}$ with the upper, transversal hole band, $Y z / Y(X z / X)$ in the $X(Y)$ direction. The hybridization between the transversal hole band and the downwards-dispersing pure $X Y$ band vanishes at $\overline{\mathrm{M}}$, but increases linearly with the distance from $\overline{\mathrm{M}}$, and with a slope proportional to the $p d \pi$-like hopping integral between the $X Y$ and $Y$ orbitals. This means that, if at $\overline{\mathrm{M}}$, the $X Y$ level at $0.6 \mathrm{eV}$ could be lowered by 0.4 $\mathrm{eV}$ such as to become degenerate with the degenerate top of the hole bands, then the transverse hole band and the $X Y$ band would form a Dirac cone. The trace of this cone can still be seen in Fig.7, in particular at the low-energy edge of the fat $X Y$ band. If the singly degenerate $X Y$ level at $\bar{M}$ had been below the degenerate $Y z / Y$ level, then this lowest level would be the singly-degenerate top of an $X Y$-like hole band and the higher-lying, doubly-degenerate level would be the bottom of the transversal $Y z / Y$ electron band. The degenerate partner does not hybridize with $X Y / Y$ and is therefore independent of the position of the $X Y$-band. Hence, the actual band structure of the $\mathrm{LaOFeAs}$ has the $X Y$ and $Y z / Y$ levels, at respectively 0.6 and $0.2 \mathrm{eV}$, inverted.

The model Hamiltonian for a Dirac cone is:

$$
H(k) \approx\left(\begin{array}{rr}
-\frac{1}{2} g & 0 \\
0 & \frac{1}{2} g
\end{array}\right)+\left(\begin{array}{cc}
\frac{\tau k^{2}}{m_{1}} & v k \\
v k & \frac{\tau k^{2}}{m_{2}}
\end{array}\right),
$$

where for the above-mentioned example $k$ is the distance from $\overline{\mathrm{M}}$. The zero of energy is midway between the two $\bar{M}$ levels, $X Y$ and $t / p$, which are separated by $g$. In view of the approximately circular shape and isotropic $X Y$ character of the transversal (outer) $\bar{M}$-centered hole sheet seen in Fig. 8 , the isotropic $2 \times 2$ Hamiltonian (3) is a reasonable representation and may be obtained by limiting $\mathrm{k}$ to one of the four $\overline{\mathrm{M}} \bar{\Gamma}$ directions where only three transversal orbitals can mix (Fig.22], and then downfolding the transversal $p$ orbital, i.e. $Y$ if $k$ is along $X$. Since the unhybridized $X Y$ band disperses downwards towards $\bar{\Gamma}$, its 

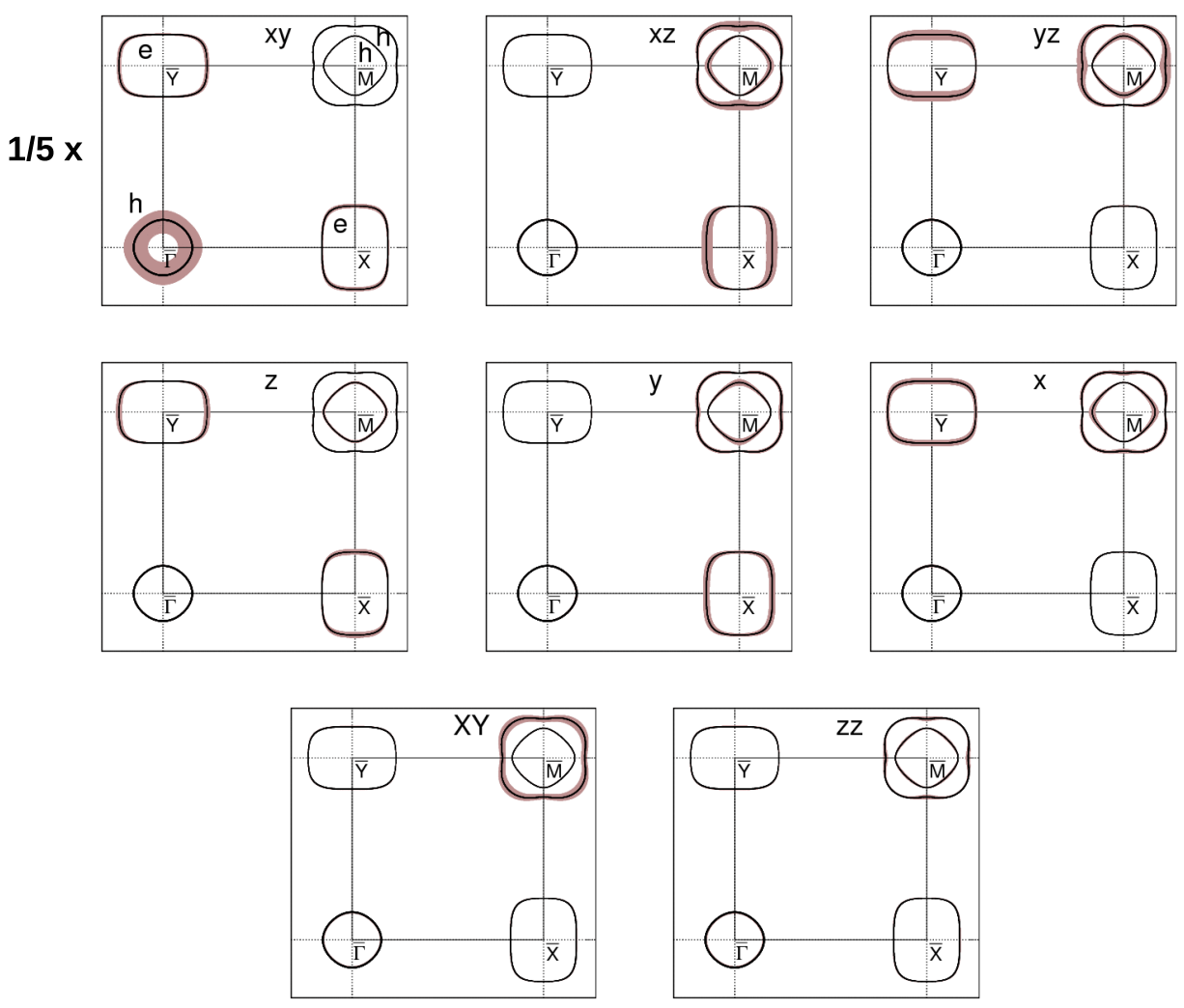

Fig. 8 Fermi surface for LaOFeAs (see also Fig. 2). The fatness was obtained as in Fig.7 7 and thus gives the orbital weight times the inverse of the Fermi velocity projected onto the plane. The fatness of the dominating $x y, x z$, and $y z$ orbitals have been reduced by a factor 5. Here, $h$ and $e$ refer to, respectively, hole and electron sheets.

mass, $m_{2}$, is negative, and since the unhybridized transversal band disperses upwards, its mass, $m_{1}$, is positive. Finally, the coupling $v k$ is proportional to the product of the $Y z-Y$ hybridization at $\bar{M}$, which is in fact responsible for moving the pure $Y z$ band up by $0.4 \mathrm{eV}$, and the $X Y-Y$ hybridization, which is proportional to $k$. These hybridizations are clearly seen in the pictures of the $d$ Wannier orbitals in Fig[5 For this incipient $X Y-Y z / Y$ Dirac cone in LaOFeAs, $g=0.4 \mathrm{eV}, m_{2}=-1.4, m_{1}=1.4$, and $v \approx$ $0.5 \mathrm{eV} a=1.4 \mathrm{eV} \AA=1.4 c / 1973$, i.e. about thousand times less than the velocity of light, $c$. Now, for $k \gg \frac{1}{2} g / v=0.4 \sim|\bar{\Gamma} \bar{M}| / 10$, the Hamiltonian (3) yields the cone: $\varepsilon(k)= \pm v k$, and for $k \ll \frac{1}{2} g / v$, it yields parabolic $Y z / Y$ and $X Y$-like bands gapped by $g$ and with inverse masses given by respectively

$$
\frac{1}{m_{1}}-\frac{v}{g}=\frac{1}{1.4}-\frac{0.5}{0.4} \sim \frac{1}{-2} \quad \text { and } \quad \frac{1}{m_{2}}+\frac{v}{g}=-\frac{1}{1.4}+\frac{0.5}{0.4} \sim \frac{1}{2} .
$$

Later in this paper, we shall meet not only incipient- but real Dirac cones.

The final gap needed to complete the $d^{6}$ gap in the central part of the $\bar{\Gamma} \bar{X} \bar{M} \bar{Y}$ square is the one produced by the avoided crossings along $\bar{M} \bar{\Gamma}$ of the downwards-dispersing upper $z / x y$ band with the upwardsdispersing upper, longitudinal $X z / X$ band. Here again, none of these bands are allowed to hybridize at $\overline{\mathrm{M}}$, and the matrix elements between them increase linearly with the distance from $\bar{M}$ in the $X$ direction. Specifically, the $p d$ matrix elements $X z-z, X-z$, and $X-x y$ are all linear in $k$. Also the $z z$ band at $-0.6 \mathrm{eV}$ 
mixes in, with the weak 2nd-nearest neighbor $d d$ hopping integral between $X z$ and $z z$ orbitals providing the slope of the linear matrix element. At $\overline{\mathrm{M}}$, the $z / x y$ and $X z / X$ levels are thus inverted, but by being at respectively 0.9 and $0.2 \mathrm{eV}$, they are too far apart to make the Dirac cone visible in Fig 7 This will however change when, in the following section, we consider other materials and include the $k_{z}$-dispersion. In conclusion, the $d^{6}$ pseudogap is caused by the $X Y$ and uppermost $z / x y$ levels being above the degenerate $t / p$ levels at $\bar{M}$. Had the opposite been the case, a situation with the two $t / p$ bands entirely above the three $z / x y$ and $e$ bands, i.e. that of a $d^{6}$ insulator, could be imagined.

Having sorted out the intricacies of the band structure and thereby understood the subtle origins of the $\overline{\mathrm{X}}$-centered electron pockets and the $d^{6}$ pseudogap, we shall finally return to the $\overline{\mathrm{M}}$-centered hole pockets using Figs.2,7, and 8 , These hole pockets have fairly complicated shapes and orbital characters. Although the $t$ character dominates, $p$ hybridization pushes the top of the band up by $0.4 \mathrm{eV}$, to $0.2 \mathrm{eV}$ above the Fermi level, as has been mentioned before. Departing from $\bar{M}$, the two bands split into a steep one with relative mass numerically smaller than one and a shallow one with mass numerically larger than one. They give rise to respectively the inner and the outer hole pockets. As long as the character of the band is predominantly $t$-like, the steeper, inner band will be the one for which the $d d \pi$ hopping is along the vector distance from $\bar{M}$, that is, the longitudinal band. Accordingly, we see in Fig.7from $\overline{\mathrm{M}}$ to $\overline{\mathrm{X}}$, the $y z$-like band stay intact and disperse strongly downwards. From $\bar{M}$ towards $\bar{\Gamma}$, we see the $X z$-like band disperse downwards and stay intact until it suffers an avoided crossing with the $z z$ band. The inner band is in fact steeper towards $\bar{\Gamma}$ than towards $\overline{\mathrm{X}}$ and $\overline{\mathrm{Y}}$, and this is partly because the $p$ hybridization of the longitudinal band has a node along $\bar{M} \bar{\Gamma}$, as can be seen for the $X z$-like band in Fig. 7 The further reason for the small mass of the inner hole pocket is the gapped Dirac cone formed with the $0.7 \mathrm{eV}$ higher-lying $z / x y$ band. The outer, transversal hole band has a large mass not given by the weak $d d \delta$ hopping integral, but as discussed above in connection with Eq. (3), by its hybridization proportional to $k$, with the $g=0.4 \mathrm{eV}$ higher-lying $X Y$ band. Along $\bar{M} \bar{\Gamma}$ this transversal hole band is mainly $x z / x$ hybridizing proportional to $k$, not only with $X Y$ but also with $z z$. The latter gives the anisotropy seen in Fig. 8 .

In the next section we shall see how these details are modified by the material-dependent height of As above the Fe plane and interlayer coupling.

\section{Influence of As height and interlayer hopping}

Until now we have discussed the generic 2D band structure for an isolated FeAs layer. This band structure was obtained by (i) downfolding the proper 3D bands of $\mathrm{LaOFeAs}$ with $\mathbf{k}$ in the small $\mathrm{BZ}$ to a $16 \times 16 p d \mathrm{~TB}$ Hamiltonian, (ii) neglecting the interlayer hoppings and (iii) reducing the resulting Hamiltonian to an $8 \times 8$ by transformation to the glide-mirror Bloch representation with $\mathrm{k}$ in the large BZ. The understanding of this relatively simple, generic, 2D band structure obtained in Sect.3.3 enables us now to explain the materialdependent, complicated, 3D bands obtained by standard DFT calculations in the small BZ. Specifically, we shall present and discuss the 3D band structures of simple-tetragonal (st) $\mathrm{LaOFeAs}$ and SmOFeAs in Fig. 9 and in Sect.4.1 mentioning those of FeTe and LiFeAs en passant, and then in Sect.4.2. move on to the band structures of body-centered tetragonal (bct) $\mathrm{BaFe}_{2} \mathrm{As}_{2}$ and $\mathrm{CaFe}_{2} \mathrm{As}_{2}$, in the normal as well as the collapsed phase. The band structure of bct $\mathrm{BaRu}_{2} \mathrm{As}_{2}$ will finally be mentioned. The interlayer hopping is mainly between As $z$ orbitals. In the st LnOFeAs materials this hopping is fairly weak and the material dependence of the band structures is caused more by the varying height of As above the Fe plane than by interlayer hopping. This we shall see in Sect.4.1 For st FeTe and LiFeAs, and in particular for the bct materials, the interlayer hopping is dominating, and since its effects are non-trivial, we have derived the formalism and shall present it in Sect.4.2. It turns out that the folding of the bands into the small BZ and subsequent interlayer hybridization at general $\mathbf{k}$-points cause many bands to have nearly linear dispersions and in some cases to form full Dirac cones. 

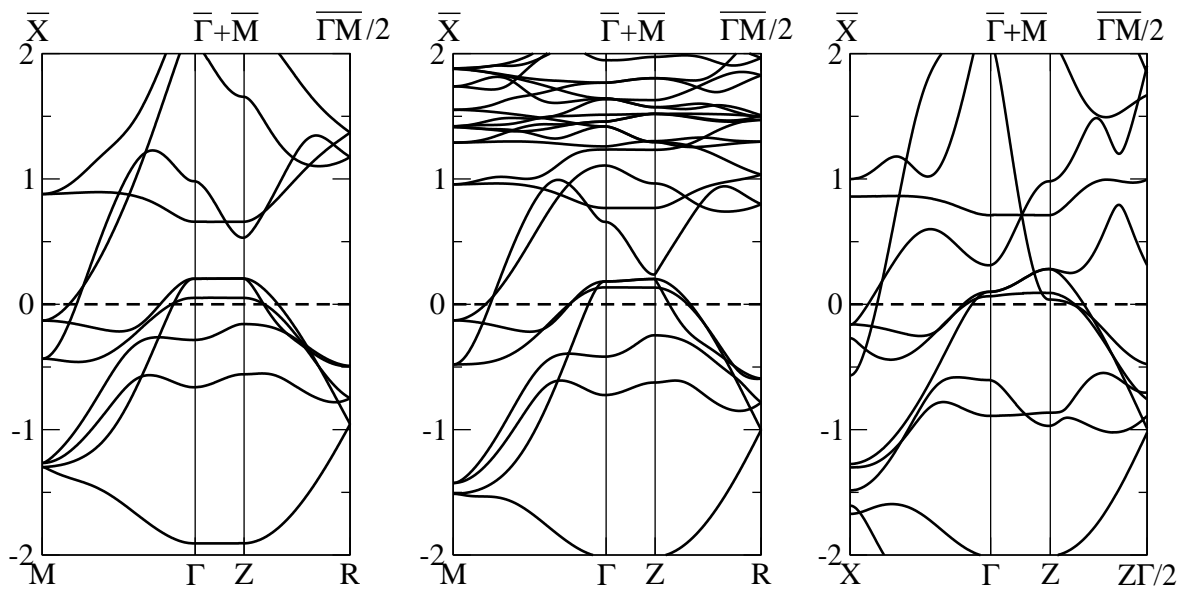

Fig. 9 3D band structures of simple tetragonal LaOFeAs (left) and SmOFeAs (middle), as well as body-centered tetragonal $\mathrm{BaFe}_{2} \mathrm{As}_{2}$ (right). For the two former, the $\mathrm{BZ}$ is a rectangular box whose cross-section is the 2D folded-in zone shown by the dashed lines in Fig. 2 The $\mathrm{M} \Gamma$-line is in the $k_{z}=0$ plane, the $\Gamma Z$-line is along the $k_{z}$-direction, and the ZR-line is in the $k_{z}=\pi / c$ plane. The 2D notation for the projection onto the $\left(k_{x}, k_{y}\right)$-plane is given on the top. The $\mathrm{BaFe}_{2} \mathrm{As}_{2}$ band structure is plotted along those same lines, now labelled $\mathrm{X} \Gamma, \Gamma \mathrm{Z}$, and $\mathrm{Z} \Gamma / 2$, as may be seen from Fig. 10] The computational scheme was GGA-LAPW ([80]).

\subsection{Simple tetragonal LnOFeAs, FeX, and LiFeAs}

Since in these st crystals, the FeAs and LnRO layers (see Fig. 1) are simply translated in the $z$-direction by a multiple of $c$ and then stacked on top of each other, the primitive translations in real and reciprocal space are respectively

$$
\left(\begin{array}{c}
\mathbf{T}_{1} \\
\mathbf{T}_{2} \\
\mathbf{T}_{3}
\end{array}\right)=\left(\begin{array}{rrr}
1 & 1 & 0 \\
-1 & 1 & 0 \\
0 & 0 & c
\end{array}\right)\left(\begin{array}{l}
\mathbf{x} \\
\mathbf{y} \\
\mathbf{z}
\end{array}\right) \text { and }\left(\begin{array}{l}
\mathbf{g}_{1} \\
\mathbf{g}_{2} \\
\mathbf{g}_{3}
\end{array}\right)=\left(\begin{array}{rrr}
\pi & \pi & 0 \\
-\pi & \pi & 0 \\
0 & 0 & \frac{2 \pi}{c}
\end{array}\right)\left(\begin{array}{l}
\mathbf{x} \\
\mathbf{y} \\
\mathbf{z}
\end{array}\right)
$$

The 3D BZ is therefore simply a rectangular box whose cross-section is the 2D zone, folded-in as shown by the dashed lines in Fig. 2. The midpoints of the vertical faces, $\frac{1}{2} \mathbf{g}_{1}$ and $\frac{1}{2} \mathbf{g}_{2}$, are labelled $\mathrm{X}=\left(\frac{1}{2} \overline{\mathrm{M}}, k_{z}=0\right)$, those of the vertical edges, $\frac{1}{2}\left(\mathbf{g}_{1} \pm \mathbf{g}_{2}\right)=\pi \mathbf{y}$ and $\pi \mathbf{x}$, are labelled $\mathrm{M}=\left(\overline{\mathrm{Y}}, k_{z}=0\right)$ and $(\overline{\mathrm{X}}, 0)$, those of the horizontal faces, $\pm \frac{1}{2} \mathbf{g}_{3}$, are labelled $\mathrm{Z}=\left(\bar{\Gamma}, \pm \frac{\pi}{c}\right)$, those of the horizontal edges, $\pm \frac{1}{2}\left(\mathbf{g}_{1}+\mathbf{g}_{3}\right)$ and $\pm \frac{1}{2}\left(\mathbf{g}_{2}+\mathbf{g}_{3}\right)$, are labelled $\mathrm{R}=\left(\frac{1}{2} \bar{M}, \pm \frac{\pi}{c}\right)$, and those of the corners, $\frac{1}{2}\left( \pm \mathbf{g}_{1} \pm \mathbf{g}_{2} \pm \mathbf{g}_{3}\right)$, are labelled $\mathrm{A}=\left(\overline{\mathrm{Y}}, \pm \frac{\pi}{c}\right)$ and $\left(\overline{\mathrm{X}}, \pm \frac{\pi}{c}\right)$.

In order to compare with our familiar 2D bands in Fig. 4, we first consider them along $\overline{\mathrm{X}} \overline{\mathrm{M}}$, where they are the same as along $\overline{\mathrm{Y}} \overline{\mathrm{M}}$, and then then translate $\overline{\mathrm{Y}} \overline{\mathrm{M}}$ by $-\mathrm{g}_{2}$ to $\overline{\mathrm{X}} \bar{\Gamma}$, which is $\mathrm{M} \Gamma$ in Fig. 9 . Now we can easily recognize the $\overline{\mathrm{M}}$-centered, doubly-degenerate top of the $t$-like hole bands, the above-lying $X Y$ and $z / x y$ bands, and the $z z$ band at $-0.6 \mathrm{eV}$. Next, we consider the $\bar{\Gamma} \overline{\mathrm{X}}$ bands in Fig. 4 and translate this line by $-\mathbf{g}_{2}$ to $\overline{\mathrm{M}} \overline{\mathrm{Y}}$, which is $\Gamma \mathrm{M}$ in Fig. 9 . This time, we recognize the $\bar{\Gamma}$-centered $x y$ hole band, the $z z / z$ band at $-0.4 \mathrm{eV}$, and the $\bar{\Gamma}$-centered bottom of the $X Y$ band at $-1.8 \mathrm{eV}$. Near $\mathrm{M}=\overline{\mathrm{X}}$ and $\overline{\mathrm{Y}}$, we also recognize the bands responsible for the $\overline{\mathrm{X}}$-centered electron super-ellipse, along the direction towards $\bar{\Gamma}$ and as well as towards $\bar{M}$. Finally, we consider the $\bar{\Gamma} \bar{M}$ bands in Fig. 4 and superpose those translated by $\mathbf{g}_{1}$, to $\bar{M} \bar{\Gamma}$, onto them. Those bands are symmetric around $\bar{M} / 2$, and their first half, from $\bar{\Gamma}$ to $\bar{M} / 2$, is placed from $\mathrm{Z}$ to $\mathrm{R}$ in Fig.9 Here again, we can easily recognize the bands. After having obtained an understanding of the $3 \mathrm{D}$ band structure of LaOFeAs from merely placing $\varepsilon_{\alpha}\left(\overline{\mathbf{k}}+\mathbf{g}_{2}\right)$ on top of $\varepsilon_{\alpha}(\overline{\mathbf{k}})$, we now search -and subsequently explain - the effects of interlayer hopping.

$k_{z}$ is 0 along $\mathrm{M} \Gamma$ and $\pi / c$ along ZR. Along the vertical path $\Gamma \mathrm{Z}$, we see the $k_{z}$-dispersion at $\overline{\mathrm{M}}$ and $\bar{\Gamma}$. From this, it may be realized that only bands with As $z$ character disperse significantly with $k_{z}$, i.e. that the 
interlayer hopping proceeds mostly from As $z$ to As $z$. The bands seen to disperse in Fig.9 are the upper $z / x y$ band near $\overline{\mathrm{M}}$ and the upper $z z / z$ band near $\bar{\Gamma}$ (Fig. (7). This interlayer hopping simply modulates the energy of the $z$ orbital, $\epsilon_{z}\left(k_{z}\right)=t^{\perp} \cos c k_{z}$. Now we see something very interesting: For $k_{z}$ near $\pi / c$, the upper $z / x y$ band has come so close to the top of the $\overline{\mathrm{M}}$-centered hole pockets that the inner, longitudinal band takes the shape of a Dirac cone over an energy region of $0.4 \mathrm{eV}$ around the Fermi level. The inner hole cylinder, as well as its radius, thus become warped due to this incipient Dirac cone. This is even more pronounced for SmOFeAs because here, the $z / x y$ band lies nearly $0.3 \mathrm{eV}$ lower than in LaOFeAs, in fact so low that the $2 \mathrm{D}$ band in the $k_{z}=\pi / c$ plane is nearly a complete Dirac cone at $0.2 \mathrm{eV}$ and with slope $v \sim 0.3 \mathrm{eV} \cdot a$ (see expression (3)). To bring the Fermi level up to the cusp would, however, require electron doping beyond $30 \%$.

The reason why the $z / x y$ band lies lower in SmOFeAs than in LaOFeAs is that As lies higher above Fe $(\eta=0.98)$ in the former than in the latter compound $(\eta=0.93)$. The $z$-xy hybridization is therefore smaller, and that moves the upper $z / x y$ band down at $\bar{M}$. This is clearly seen along all directions in Fig.9. but whereas this flattening of the upper $z / x y$-like band increases the mass at the $\overline{\mathrm{X}}$-centered electron pocket towards $\overline{\mathrm{M}}$ and makes it more $d$-like, it decreases the mass of the inner $\overline{\mathrm{M}}$-centered $t / p$-like hole pocket due to the incipient Dirac cone. Increasing $\eta$, generally decreases the $p d$ hybridization, whereby $p d$ antibonding levels move down in energy with respect to those of pure $d$ character and become more $d$-like. Important effects of this are the lowering of the top of the $t / p$ hole band at $\bar{M}$ with respect to that of the $x y$ hole band at $\bar{\Gamma}$ and the lowering of the bottom of the $x y$-like electron band with respect to the pure $x z$ level at $\overline{\mathrm{X}}$. These changes are clearly seen in Fig. 9 . With the Fermi level readjusted, the size of the $\bar{\Gamma}$-centered $x y$ hole sheet is increased for SmOFeAs and is now similar to that of the outer $\bar{M}$-centered hole sheet. Finally, we may note that the decreased $t / p$ hybridization at $\bar{M}$ decreases the coupling linear in $k$ to the $X Y$ band, so that this band becomes less steep in the Sm than in the La compound.

Of all known iron-based superconductors, $\mathrm{SmOFeAs}$ has the highest $T_{c} \max (55 \mathrm{~K})$ and the most regular $\mathrm{FeAs}_{4}$ tetrahedron, i.e. its $\eta$ is closest to 1 . For nearly all Fe-based superconductors, $T_{c}$ max versus $\eta$ seems to follow a parabolic curve, [13] a correlation which has been extensively studied, but is not understood. For $\mathrm{LaOFeAs}, T_{c \text { max }}=27 \mathrm{~K}$.

Also LiFeAs and the iron chalcogenides, FeX, have the st structure and calculations [23, 34, 35] yield: $\eta=1.12$ for LiFeAs, while for $\mathrm{X}=\mathrm{S}, \mathrm{Se}, \mathrm{Te}: \eta=0.87,0.97,1.16$, respectively [35]. In LiFeAs and FeTe, the upper $z / x y$ band thus sits considerably lower in energy. Moreover, since in LiFeAs the perpendicular As $z$ hopping is enhanced by hopping via $\mathrm{Li} s$, and since the perpendicular Te $5 p_{z}$ hopping is stronger than the As $4 p_{z}$ hopping in LnOFeAs, the $z / x y$ band disperses 3 and 4 times more along $\Gamma Z$ in respectively $\mathrm{LiFeAs}$ and FeTe, than in LnOFeAs. As a consequence, the $z / x y$-like band crosses the degenerate $t / p$ band already when $k_{z} \sim \pi / 2 c$, and here, it forms a Dirac cone with the inner, longitudinal $t / p$ band, at 0.1 $\mathrm{eV}$ above the Fermi level in LiFeAs and at $0.2 \mathrm{eV}$ in FeTe. For $\pi / 2 c \lesssim k_{z} \lesssim 3 \pi / 2 c$, the band which at $\overline{\mathrm{M}}$ has longitudinal $t / p$ character disperses upwards and the other band, which at $\overline{\mathrm{M}}$ has $z / x y$ character, downwards. Accordingly, the inner hole sheet of the Fermi surface is not a cylinder, but extends merely a bit further than from $-\pi / 2 c$ to $\pi / 2 c$ where the $z / x y$ band along $\Gamma Z$ dips below the Fermi level. The mass of this sheet vanishes when $k_{z}$ is at the Dirac value, $\sim \pi / 2 c$. Here, the slope of the cone in the $\left(k_{x}, k_{y}\right)$ plane is $v \sim 0.5 \mathrm{eV} \cdot a$. The $\bar{\Gamma}$-centered $x y$ pocket is a straight cylinder, whose cross-section in FeTe has about the same size as that of the outer $\overline{\mathrm{M}}$-centered $t / p$ hole sheet at $k_{z}=0$, i.e. like in SmOFeAs, and in $\mathrm{LiFeAs}$ is even a bit larger. LiFeAs is a non-magnetic superconductor with $T_{c}=18 \mathrm{~K}$.

\subsection{Body-centered tetragonal $\mathrm{BaFe}_{2} \mathrm{As}_{2}, \mathrm{CaFe}_{2} \mathrm{As}_{2}$, and $\mathrm{BaRu}_{2} \mathrm{As}_{2}$}

In the body-centered tetragonal (bct) structure, the FeAs layers are translated by $\mathrm{x}$ before they are stacked on top of each other. This means that the As atoms of adjacent layers are directly on top of each other. Moreover, the interlayer As-As distance, $d=379 \mathrm{pm}$ is about the same as the intralayer As-As distances, $\sqrt{2} a=396 \mathrm{pm}$ and $\sqrt{2} a \eta$. It is therefore conceivable that the interlayer hopping vertically from As $z$ to As $z(p p \sigma)$ is very strong. This is in fact the reason for the $2 \mathrm{eV}$ dispersion seen along $\Gamma \mathrm{Z}$ in the band structure of $\mathrm{BaFe}_{2} \mathrm{As}_{2}$ on the right-hand side of Fig. 9 Since $\eta=0.97$ for $\mathrm{BaFe}_{2} \mathrm{As}_{2}$, the position of this $z$-like band 
is not as low as in $\mathrm{FeTe}$, but more like in SmOFeAs. At $k_{z} \approx 3 \pi / 4 c$, the band crosses the degenerate $t / p$ band and forms a Dirac cone with its longitudinal branch in the $\left(k_{x}, k_{y}\right)$-plane, as we shall see explicitly later. Note that the longitudinal branch disperses downwards from $\Gamma$ towards $\mathrm{X}$ in the $k_{z}=0$ plane, but upwards from $\mathrm{Z}$ in the $k_{z}=\pi / c$ plane.

$\mathrm{Ba}$ is intercalated in the holes between the neighboring As sheets and thus has 8 nearest As neighbors. Also Ba orbitals can be vehicles for interlayer coupling and, in fact, a Ba $5 d_{x z / y z}$ band lying above the frame of Fig.9 9 repels the top of the doubly degenerate $t / p$ band near $\Gamma$ with the result that there, the latter is only slightly above the top of the dispersionless $\bar{\Gamma} x y$ band, whereas at $\mathrm{Z}$, it is $0.2 \mathrm{eV}$ above. Clearly visible in the figure is also a $\mathrm{Ba} 5 d_{x y}$ band starting at $1.0 \mathrm{eV}$ at $\mathrm{X}$ and then dispersing downwards towards $\Gamma$, which is reached at $0.3 \mathrm{eV}$ after an avoided crossing with the $y / x z$ band decreasing from its maximum at $\Gamma$. From $\Gamma$ towards $\mathrm{Z}$, the $5 d_{x y}$ band then disperses upwards to $1.0 \mathrm{eV}$ and, from there, continues in the $k_{z}=\pi / c$ plane towards $\Gamma$, but soon suffers an avoided crossing with the hybridized $z / x y$-longitudinal- $t / p$ band.

But before we continue our discussion of the $\mathrm{BaFe}_{2} \mathrm{As}_{2}$ bands we need to write down a formalism for the interlayer coupling which is strong -and poorly understood-in the bct structure.

We start from the 2D Bloch waves, $|\mathbf{r} ; \alpha, \overline{\mathbf{k}}\rangle$, of a single FeAs layer with $\alpha$ labelling the state (e.g. the band) and $\overline{\mathbf{k}}$ the irreducible representation of the glide-mirror group. These 2D Bloch waves are expressed as linear combinations of localized Wannier orbitals. For the 3D crystal, we now use its out-of-plane translations, $n_{3} \mathbf{T}_{3}$, to stack the $2 \mathrm{D}$ Bloch waves in the 3 rd direction and form the corresponding Bloch sums:

$$
|\mathbf{r} ; \alpha, \mathbf{k}\rangle=\sum_{n_{3}=0, \pm 1, \ldots}\left|\mathbf{r}-n_{3} \mathbf{T}_{3} ; \alpha, \overline{\mathbf{k}}\right\rangle \exp \left(i n_{3} \mathbf{T}_{3} \cdot \mathbf{k}\right),
$$

which we shall then use as basis functions. Here and in the remainder of this chapter, an overbar is placed on the $2 \mathrm{D}$ Bloch vector in order to distinguish it from the $3 \mathrm{D}$ one, $\mathbf{k} \equiv \overline{\mathbf{k}}+k_{z} \mathbf{z}$.

Since in the $b c t$ structure, the As atoms in a top sheet are vertically below those in the bottom sheet of the layer above, the corresponding vertical interlayer hopping via As $z$ is particularly simple and strong, so we first specialize to this case. The bct primitive translations in real and reciprocal space are respectively:

$$
\left(\begin{array}{l}
\mathbf{T}_{1} \\
\mathbf{T}_{2} \\
\mathbf{T}_{3}
\end{array}\right)=\left(\begin{array}{ccc}
1 & 1 & 0 \\
-1 & 1 & 0 \\
-1 & 0 & c
\end{array}\right)\left(\begin{array}{l}
\mathbf{x} \\
\mathbf{y} \\
\mathbf{z}
\end{array}\right) \text { and }\left(\begin{array}{l}
\mathbf{g}_{1} \\
\mathbf{g}_{2} \\
\mathbf{g}_{3}
\end{array}\right)=\left(\begin{array}{ccc}
\pi & \pi & \frac{\pi}{c} \\
-\pi & \pi & -\frac{\pi}{c} \\
0 & 0 & \frac{2 \pi}{c}
\end{array}\right)\left(\begin{array}{l}
\mathbf{x} \\
\mathbf{y} \\
\mathbf{z}
\end{array}\right)
$$

where $c$ is the distance between the FeAs layers in units of the nearest-neighbor Fe-Fe distance, i.e. half the bct c-lattice constant. The bct Brillouin zone and the stacking between neighboring Wigner-Seitz cells (BZs) of the reciprocal g-lattice is shown in Fig. 10, Using that $\mathbf{T}_{3} \cdot \mathbf{k}=k_{x}+c k_{z}$, it is now a simple matter to form the 3D Bloch sums (5). For two states $\alpha$ and $\beta$ with the same $\overline{\mathbf{k}}$, the interlayer coupling caused by the vertical $z-z$ hopping is easily found as:

$$
\langle\alpha, \mathbf{k}|\hat{H}| \beta, \mathbf{k}\rangle_{\text {inter }}=t^{\perp} c_{z, \alpha}^{*}(\overline{\mathbf{k}}) c_{z, \beta}(\overline{\mathbf{k}}) \cos c k_{z},
$$

where $t^{\perp}$ is the $p p \sigma$ hopping integral $(\leq 0)$ between an As $z$ orbital in the top sheet to the As $z$ orbital vertically above, in the bottom sheet of the next layer. $c_{z, \beta}(\overline{\mathbf{k}})$ is the eigenvector coefficient to the As $z$ orbital in the $2 \mathrm{D} \beta$-state. Note that we have not missed a factor 2 in (7), because only one lobe of the $z$ orbital is used for interlayer coupling. Now, from the mere knowledge of a $\overline{\mathbf{k}}$-function in a single -bottom or top- As sheet, the $\overline{\mathbf{k}}$ and $\overline{\mathbf{k}}+\overline{\mathbf{g}}_{1}\left(\right.$ or $\overline{\mathbf{k}}+\overline{\mathbf{g}}_{2}$ ) translational states are indistinguishable. Their difference is that they have opposite parity upon the glide-mirror interchanging the top and bottom sheets. Interlayer hopping can therefore mix states with $\overline{\mathbf{k}}$ and $\overline{\mathbf{k}}+\overline{\mathbf{g}}_{1}$. For the corresponding interlayer coupling, off-diagonal in the 2D Bloch vector, we then find :

$$
\left\langle\alpha, \mathbf{k}|\hat{H}| \beta, \mathbf{k}+\mathbf{g}_{1}\right\rangle_{\text {inter }}=-i t^{\perp} c_{z, \alpha}^{*}(\overline{\mathbf{k}}) c_{z, \beta}\left(\overline{\mathbf{k}}+\overline{\mathbf{g}}_{1}\right) \sin c k_{z}
$$




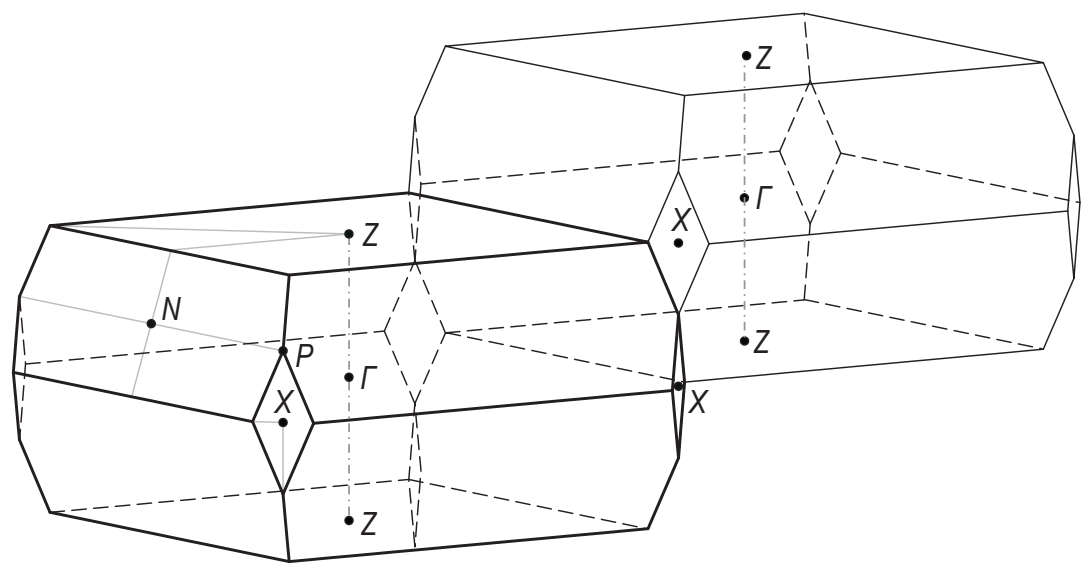

Fig. 10 Central bct Brillouin zone and the one translated by the reciprocal-lattice vector $\mathbf{g}_{1}=\pi \mathbf{x}+\pi \mathbf{y}+\pi \mathbf{z} / c$, see Eq. (6). $\Gamma$ is the center of the zone, $\mathrm{Z}= \pm \mathbf{g}_{3} / 2= \pm \pi \mathbf{z} / c$ are the centers of the 2 horizontal faces, $\mathrm{N}$ are those of the 8 large slanting faces, e.g. $\mathbf{g}_{1} / 2, X$ are the centers of the 4 vertical faces, e.g. $\left(\mathbf{g}_{1}-\mathbf{g}_{2}+\mathbf{g}_{3}\right) / 2=\pi \mathbf{x}$ and $\left(\mathbf{g}_{1}+\mathbf{g}_{2}\right) / 2=\pi \mathbf{y}$, and $\mathrm{P}$ are the 8 corners between the vertical $\mathrm{X}$ neighbors, e.g. $\pi \mathbf{x}+\pi \mathbf{z} / 2 c$. Note that $\mathrm{Z}$ in the zone translated by $\mathbf{g}_{1}$ is $\mathbf{g}_{1}-\mathbf{g}_{3} / 2=\pi \mathbf{x}+\pi \mathbf{y}$.

where the different parities of the $\overline{\mathbf{k}}$ and $\overline{\mathbf{k}}+\overline{\mathbf{g}}_{1}$ states causes the $\sin c k_{z}$-dispersion. Finally, before coupling the $\mathbf{k}+\mathbf{g}_{1}$ state (see Fig. 10) to that with $\mathbf{k}$, the former must be brought back to the central zone, and that requires shifting $k_{z}$ in (7) back by $\pi / c$. As a consequence,

$$
\left\langle\alpha, \mathbf{k}+\mathbf{g}_{1}|\hat{H}| \beta, \mathbf{k}+\mathbf{g}_{1}\right\rangle_{\text {inter }}=-t^{\perp} c_{z, \alpha}^{*}\left(\overline{\mathbf{k}}+\overline{\mathbf{g}}_{1}\right) c_{z, \beta}\left(\overline{\mathbf{k}}+\overline{\mathbf{g}}_{1}\right) \cos c k_{z}
$$

In order to get a first feeling for this formalism, let us assume that we have nothing, but interlayer hopping. That is, we have pure $z$ states which only couple between -but not inside- the layers. The Hamiltonian for this problem with two $z$-orbitals per cell is:

$$
H(\mathbf{k})=\left(\begin{array}{cc}
t^{\perp} \cos c k_{z} & -i t^{\perp} \sin c k_{z} \\
i t^{\perp} \sin c k_{z} & -t^{\perp} \cos c k_{z}
\end{array}\right)
$$

Diagonalization yields two dispersionless bands with energy $\pm t^{\perp}$, and this is because this system without intra-layer coupling is merely an assembly of $\mathrm{As}_{2}$ dimers (dangling bonds). The same kind of thing happens at the non-horizontal boundaries of the bet BZ, where the states with energies $\varepsilon_{\alpha}(\overline{\mathbf{k}})$ and $\varepsilon_{\alpha}\left(\overline{\mathbf{k}}+\overline{\mathbf{g}}_{1}\right)$ are degenerate, because if there are no further degeneracies and if $t^{\perp}$ is so small that we only need to consider those two states, their energies simply split by $\pm t^{\perp}\left|c_{z, \alpha}(\overline{\mathbf{k}})\right|^{2}$. We thus see, that neglecting interlayer coupling does not simply correspond to taking $k_{z}=\pi / 2 c$; this merely makes the diagonal couplings vanish.

In simple tetragonal $\mathrm{FeX}$, the interlayer coupling proceeds mainly from an As $z$ orbital to its 4 nearest As $z$ orbitals in the next layer, with a hopping integral $t^{<}$. From this follows that the diagonal and offdiagonal interlayer couplings are given by respectively

$$
\begin{aligned}
\langle\alpha, \mathbf{k}|\hat{H}| \beta, \mathbf{k}\rangle_{\text {inter }} & =4 t^{\llcorner} c_{z, \alpha}^{*}(\overline{\mathbf{k}}) c_{z, \beta}(\overline{\mathbf{k}})\left(\cos k_{x}+\cos k_{y}\right) \cos c k_{z}, \\
\left\langle\alpha, \mathbf{k}|\hat{H}| \beta, \mathbf{k}+\mathbf{g}_{1}\right\rangle_{\text {inter }} & =-4 i t^{\llcorner} c_{z, \alpha}^{*}(\overline{\mathbf{k}}) c_{z, \beta}\left(\overline{\mathbf{k}}+\overline{\mathbf{g}}_{1}\right)\left(\cos k_{x}+\cos k_{y}\right) \sin c k_{z} .
\end{aligned}
$$

Since the simple tetragonal reciprocal lattice vectors $\mathbf{g}_{1}$ and $\mathbf{g}_{2}$ in Eq. (4) have no $k_{z}$ component, there is no $k_{z}$-translation leading to an Eq. (9). Instead, the prefactor $\cos k_{x}+\cos k_{y}$ provides the sign-change for 


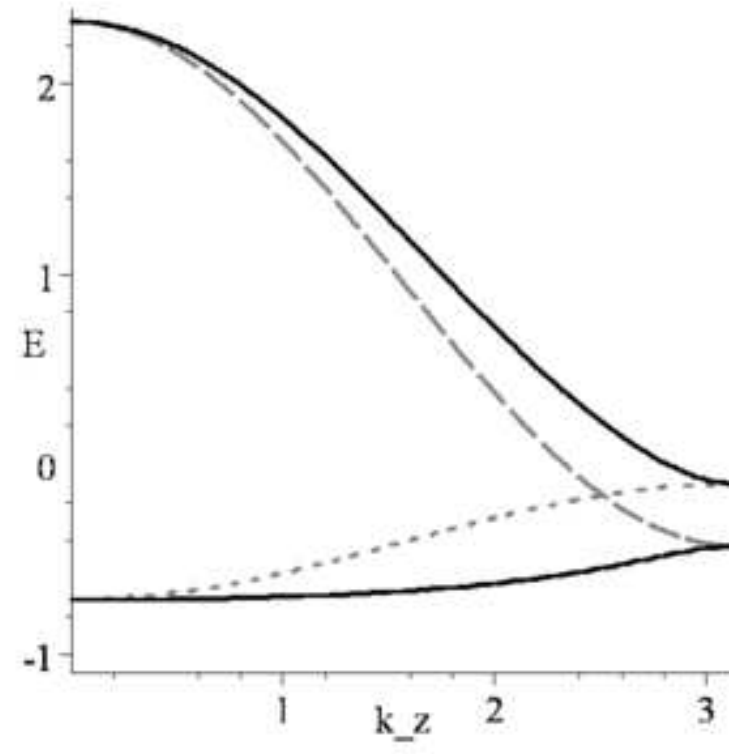

Fig. 11 Interlayer coupling of the $\left(\overline{\mathrm{M}}, k_{z}-\pi / c\right) z / x y$ (grey dashed) and the $\left(\bar{\Gamma}, k_{z}\right) z / z z$ (grey dotted) bands along $\Gamma \mathrm{Z}$ for bct $\mathrm{BaFe}_{2} \mathrm{As}_{2}$ (schematic). We used Eq.s (8) and (9) together with the 2D LaOFeAs parameters and $t^{\perp}=2 \mathrm{eV}$.

$\overline{\mathbf{k}}$ going to the next BZ. In addition, the prefactor makes the interlayer coupling vanish on the vertical faces of the 3D st BZ, that is on $\left(\overline{\mathrm{X}} \bar{Y}, k_{z}\right)$. This is different from the bct case. Finally, for the system with only $z$ orbitals and no intra-layer coupling the st fomalism yields two dispersionless bands with energies $\pm 4 t^{\angle}$ corresponding to isolated As- $\mathrm{As}_{4}$ molecules with no coupling between the 4 atoms in the same plane.

Having deepened our understanding of the interlayer coupling via the As $z$ orbitals, we can now return to our description of the bct band structures for which this interlayer hopping is particularly strong and most noticeably-gives rise to the $2 \mathrm{eV}$ dispersion of the $z$-like band seen along $\Gamma \mathrm{Z}$ for $\mathrm{BaFe}_{2} \mathrm{As}_{2}$ in Fig. 9 This dispersion is seen to be five times larger than in the LnOFeAs compounds, and it turns out that this is not even the entire interlayer dispersion, $2 t^{\perp}\left|c_{z, z / x y}(\overline{\mathrm{M}})\right|^{2}$ :

From our previous discussion of the $2 \mathrm{D}$ bands, we may recall that, at $\overline{\mathrm{M}}$, the $z$ orbital can only mix with $x y$ and the level of interest is the antibonding $z / x y$ level which in LaOFeAs is at $0.9 \mathrm{eV}$ and has about $70 \% z$ character, specifically, $c_{z, z / x y}(\overline{\mathrm{M}})=-0.83$. At $\bar{\Gamma}, z$ can only mix with $z z$ and there again, the level of interest is the antibonding $z z / z$ level, which in LaOFeAs is at $-0.4 \mathrm{eV}$ and $85 \% d$-like, $c_{z, z z / z}(\bar{\Gamma})=-0.39$. If we now just couple those two LaOFeAs antibonding bands with the bet interlayer coupling given by Eqs. (7), (8), and (97), and adjust the one parameter $t^{\perp}$ to the $\mathrm{BaFe}_{2} \mathrm{As}_{2} \Gamma \mathrm{Z}$ band, thus yielding $t^{\perp} \sim-2 \mathrm{eV}$, we get the two bands shown in Fig.11. The good agreement of the upper band with the $\Gamma \mathrm{Z}$ band in $\mathrm{BaFe}_{2} \mathrm{As}_{2}$, dispersing from 2.1 to $0.1 \mathrm{eV}$ in Fig.9 hints that this band does result from an avoided crossing of the downwards-dispersing $\overline{\mathrm{M}} z / x y$ band and the upwards-dispersing $\bar{\Gamma} z / z z$ band, such that the $0.1 \mathrm{eV}$ state at $\mathrm{Z}$ is not $\overline{\mathrm{M}} z / x y$, but $\bar{\Gamma} z z / z$. The $\overline{\mathrm{M}} z / x y$ state at $\mathrm{Z}$ must then be the top of lower band which is seen to have energy $-0.9 \mathrm{eV}$ in $\mathrm{BaFe}_{2} \mathrm{As}_{2}$ (this includes a push-down by a high-lying $\mathrm{Ba} 5 d_{z z}$ band). The bottom of the lower band is then the $\bar{\Gamma} z / z z$ state at $\Gamma$, which in $\mathrm{BaFe}_{2} \mathrm{As}_{2}$ is seen accidentally also to have energy $-0.9 \mathrm{eV}$.

After this estimate, let us briefly recall the proper way of including the interlayer $z-z$ coupling along $\Gamma Z$. First of all, from the caption to Fig. 2 we learn that $z$ can only mix with $x y$ at $\bar{M}$ and with $z z$ at $\bar{\Gamma}$. Secondly, from the 2D bands in Fig. 7 , we see that at $\bar{M}$, as well as at $\bar{\Gamma}$, the $z$-like levels are separated by as much as $4 \mathrm{eV}$. This is due to strong $p d$ hybridization; the $z, x y$ matrix element at $\bar{M}$ is $2 \mathrm{eV}$ and the $z, z z$ element at $\bar{\Gamma}$ is $1.2 \mathrm{eV}$. For an interlayer hopping, $t^{\perp}$, as large as $2 \mathrm{eV}$, we now ought to solve a $4 \times 4$ 

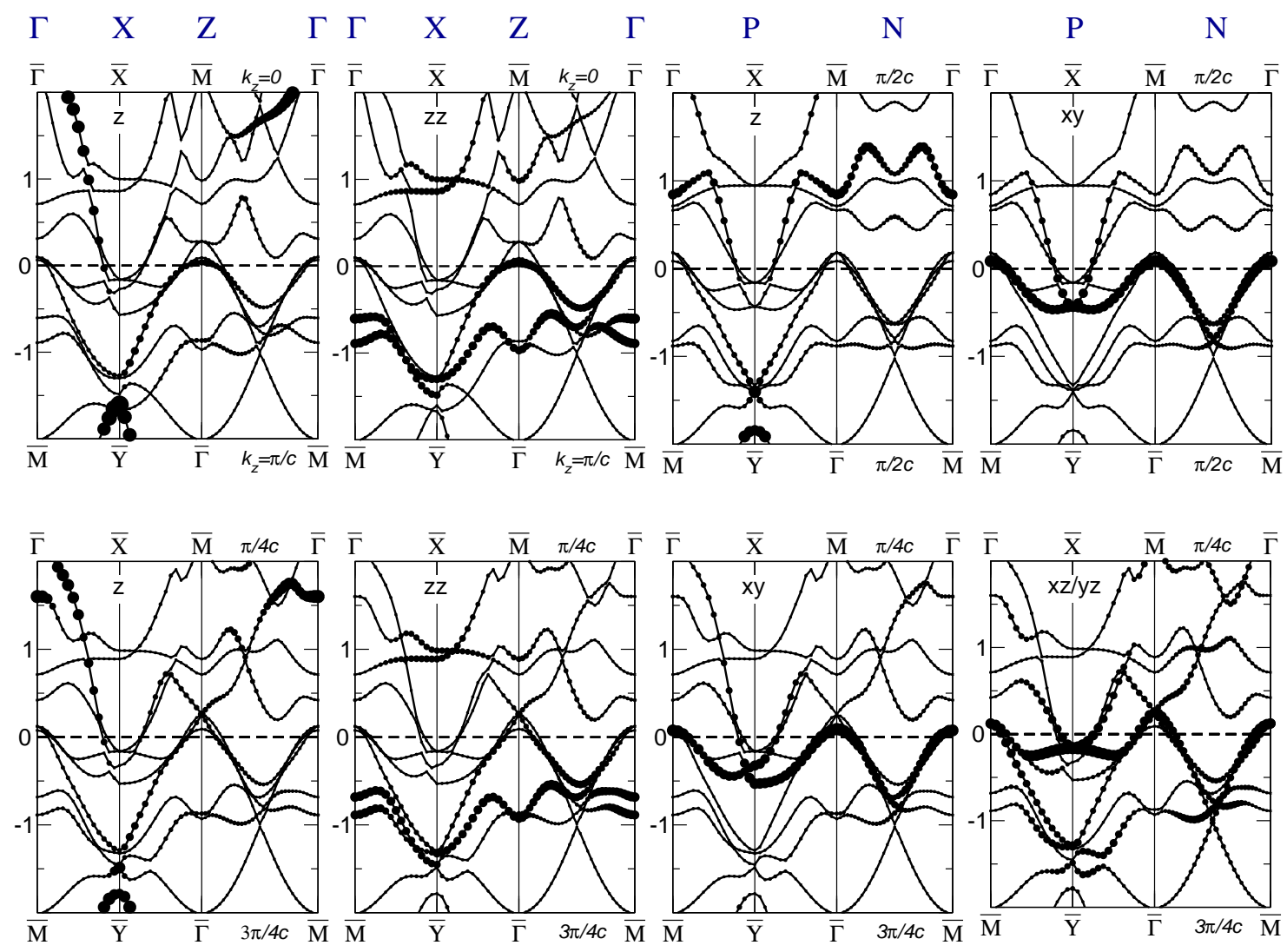

Fig. $123 \mathrm{D}$ band structure of bct $\mathrm{BaFe}_{2} \mathrm{As}_{2}$, fattened by various partial-wave characters and calculated with the GGA-LAPW method ([80]). The $\mathbf{k} \equiv\left(\overline{\mathbf{k}}, k_{z}\right)$ paths chosen are: $(\bar{\Gamma} \bar{X} \bar{M} \bar{\Gamma}, 0)=(\bar{M} \bar{Y} \bar{\Gamma} \bar{M}, \pi / c)$, in the two top left panels, $(\bar{\Gamma} \overline{\mathrm{X}} \overline{\mathrm{M}} \bar{\Gamma}, \pi / 2 c)=(\overline{\mathrm{M}} \overline{\mathrm{Y}} \bar{\Gamma} \overline{\mathrm{M}}, \pi / 2 c)$ in the two top right panels, and $(\bar{\Gamma} \overline{\mathrm{X}} \overline{\mathrm{M}} \bar{\Gamma}, \pi / 4 c)=(\overline{\mathrm{M}} \overline{\mathrm{Y}} \bar{\Gamma} \overline{\mathrm{M}}, 3 \pi / 4 c)$ in the four bottom panels. Note that these paths extend over the two BZs shown in Fig. 10. The fatness of As $z$ has been enhanced by a factor 5 compared with those of Fe $d$ in order to account approximately for the fact that the As $z$ Wannier orbital has most of its charge density outside the atomic sphere used in the LAPW method.

eigenvalue problem (that the $d$ states at $\overline{\mathbf{k}}$ and $\overline{\mathbf{k}}+\overline{\mathbf{g}}$ are different is irrelevant). However, for producing Fig.11, we got away with neglecting the $p d$-bonding levels. On the other hand, $t^{\perp} \sim-2 \mathrm{eV}$ was obtained by fitting to bands which include the $\mathrm{Ba} 5 d_{z z}$ hybridization and this leads to an overestimation of $t^{\perp}$, as we shall see later.

We now discuss further aspects of the bct band structure computed for $\mathrm{BaFe}_{2} \mathrm{As}_{2}$. The first two top panels of Fig. 12 show respectively the As $z$ and $\mathrm{Fe} z z$ projected bands for $k_{z}=0$ and along the path $\bar{\Gamma} \overline{\mathrm{X}}$ $\bar{M} \bar{\Gamma}$, familiar from Fig.7 but labelled $\Gamma X Z \Gamma$ in the bct reciprocal lattice (Fig. 10). This path takes us from the center, $\Gamma$, of the central BZ to the center, $\mathrm{X}$, of a vertical BZ face, from there to the center, $\mathrm{Z}$, of the bottom face of the neighboring BZ, and finally back to the origin, $\Gamma$. The piece outside the central $\mathrm{BZ}$ may of course be translated back to the bottom face of the central zone by $-\mathrm{g}_{1}$, or to the top face by $\mathbf{g}_{3}-\mathrm{g}_{1}$. As a result, not only the eight $\Gamma X Z \Gamma=(\bar{\Gamma} \bar{X} \bar{M} \bar{\Gamma}, 0)$-bands, but also the eight $\Gamma X Z \Gamma=(\bar{M} \bar{Y} \bar{\Gamma} \bar{M}, \pi / c)$-bands are obtained in such a standard calculation. For $k_{z}=0$ and $\pi / c$, the $\overline{\mathbf{k}}$-states are pure because $\sin c k_{z}=0$, and the $\mathrm{Fe} z z$ projection confirms that the $0.1 \mathrm{eV}$ state at $\mathrm{Z}$ has $z z$ character.

The other band with $z z$ character, i.e. the $\overline{\mathrm{M}} z z$ band, is seen to have energy $-0.6 \mathrm{eV}$ at $\Gamma$ and $-1.0 \mathrm{eV}$ at Z. For reasons of symmetry, this band can have no As $z$ character, and nevertheless disperses with $k_{z}$ (as seen directly along $\Gamma Z$ in Fig. 9 . This is due to repulsion from the Ba $5 d_{x y}$ band. 
When, in Fig. 9 , we observed that the longitudinal branch of the doubly-degenerate $t / p$ band curves downwards at $\Gamma$ in the $k_{z}=0$ plane, but upwards at $\mathrm{Z}$ in the $k_{z}=\pi / c$ plane, both in the central BZ (Fig.10], this was seen as a consequence of the Dirac cone in the $k_{z}=3 \pi / 4 c$ plane at the crossing of the $t / p$ band with the upper $z$-like band between $\Gamma$ and Z. In the meantime, we have learned that the upper $z$-like band cannot hybridize with the $t / p$ band in the $k_{z}=\pi / c$ plane where $\sin c k_{z}=0$, because the former is a $\bar{\Gamma}$-state and the latter an $\overline{\mathrm{M}}$-state at $\mathrm{Z}$. The upwards curvature at $\mathrm{Z}$ is therefore due to repulsion from the lower $z$-like band, the $\overline{\mathrm{M}} z / x y$ band near $-0.9 \mathrm{eV}$. This repulsion is substantial and causes another, but merely incipient Dirac point at $-0.4 \mathrm{eV}$ at Z. However, as we now depart from the $k_{z}=n \pi / c$ plane, also the $\bar{\Gamma} z / z z$ band hybridizes with the longitudinal $t / p$ band -proportional to $\sin c k_{z}$ and to the horizontal distance from $\overline{\mathrm{M}}-$ and this causes a Dirac cone to be formed at the crossing of the upper $z$-like and the degenerate $t / p$ bands at $k_{z}=3 \pi / 4 c$ and $0.25 \mathrm{eV}$. At the bottom four panels of Fig. 12 we therefore show the band structure in the $k_{z}=\pi / 4 c+n \pi / c$ and $k_{z}=3 \pi / 4 c+n \pi / c$ planes extending over the two BZs, projected onto the As $z$ and relevant Fe $d$ partial waves. The Dirac cone at $(\overline{\mathrm{M}}, \pi / 4 c)$ in the $\mathrm{BZ}$ at $\mathrm{g}_{1}$ is seen to have $z, z z$, longitudinal $t / p$, and $x y$ characters in agreement with what was said above, and to have a low-energy slope and a steeper high-energy slope. The low-energy slope $v=0.4 \mathrm{eV} \cdot a$ is of course due to the hybridization of the $t / p$ band with the crossing, upper $z$-like band and the high-energy slope $v=0.8 \mathrm{eV} \cdot a$ is due to hybridization with the lower $z$-like band, which is around $-0.9 \mathrm{eV}$. (The hole band seen in Fig. 12 to have the strongest $x y$ character is irrelevant for the Dirac cone because it is the $\bar{\Gamma}$-centered hole band which, due to lack of $z$ character, cannot mix with $\mathbf{k}+\mathbf{g}_{1}$ states). As $k_{z}$ is now increased above $\pi / 4 c$, e.g. to $k_{z}=\pi / 2 c$ as shown in the last two top panels of Fig. 12, the upper $z$-like band moves above the $t / p$ band at $\bar{M}$ to $1.0 \mathrm{eV}$, whereby the longitudinal branch of the latter curves downwards, like the transversal branch. For $k_{z}=3 \pi / 4 c$, the behaviour can be seen near $\overline{\mathrm{M}}$ in the four bottom panels: The upper $z$-like band is now at $2.1 \mathrm{eV}$ and has no $z / z z$ but only $z / x y$ character. The repulsion from this band steepens the longitudinal, inner $t / p$ hole band.

The outer, transversal $t / p$ hole band attains its hole character mainly from repulsion by the $X Y$ band, as was explained in connection with Eq. (3) and seen in Fig. 7 (but excluded in Fig.(12). Finally, for $k_{z}=\pi / c$, the behaviour can be seen near $\bar{M}$ in first panel, which in fact shows what we have already observed without orbital projections on the right-hand side of Fig.9

The hole part of the Fermi surface thus has a strongly warped, cylindrical sheet, centered around the vertical $\Gamma Z$ line (see Fig. 10p. In most of the zone, this sheet has longitudinal $\bar{M} t / p$ and some $\bar{M} z / x y$ character, i.e. it is the small-mass, inner hole sheet. Going from $\Gamma$ towards $Z$, this sheet narrows down to a neck for the Dirac value, $k_{z}=k_{D z}$, where the $\bar{\Gamma} z / z z$ character starts to dominate. Finally, close to Z, the character becomes purely $\bar{\Gamma} z / z z$ and the cylinder bulges out. Unlike in FeTe and in LiFeAs, this sheet remains a cylinder because the upper $z$-like band remains above the Fermi level for all $k_{z}$. Concentric with this longitudinal $t / p$ hole cylinder is the transversal one, whose mass is dominated by its $X Y$ character. That cylinder has little warping and lies outside the longitudinal cylinder, except near $\mathrm{Z}$ where the latter bulges out. The third hole sheet has nearly pure $\bar{\Gamma} x y$ character and is a straight cylinder. Also this cylinder is centered along $\Gamma Z$, but it does not hybridize with the two $\bar{M}$ cylinders and it is as narrow as the Dirac neck of the longitudinal $t / p$ cylinder.

Next, we turn to the electron sheets, which in 2D are the $\overline{\mathrm{X}}$ and $\overline{\mathrm{Y}}$-centered super-ellipses pointing towards $\bar{M}$ (Fig.22). They are formed by the $x y$-like band together with the transverse $x z$-like band for the $\bar{X}$ sheet and with the transverse $y z$-like band for the $\bar{Y}$ sheet (see Eq. (2)). Figs. 7 and 8 show that the only part which has As $z$ character and may therefore disperse with $k_{z}$, is the one pointing towards $\overline{\mathrm{M}}$. The corresponding band is the $z / x y$ band which we studied above and which at $\overline{\mathrm{M}}$ was found to disperse by $3 \mathrm{eV}$, from $2.1 \mathrm{eV}(\Gamma)$ to $-0.9 \mathrm{eV}(\mathrm{Z})$. Being centered at respectively $\overline{\mathrm{X}}$ and $\overline{\mathrm{Y}}$, the electron cylinders are however far away from $\overline{\mathrm{M}}$, and since the $x y$ band can have no $z$ character at $\overline{\mathrm{X}}$ and $\overline{\mathrm{Y}}$, it hardly disperses with $k_{z}$ there. But the $z$ character increases linearly with the distance $k_{y}$ from $\overline{\mathrm{X}}$-and with the distance $k_{x}$ from $\overline{\mathrm{Y}}$ - towards $\overline{\mathrm{M}}$, and so does the upwards $k_{z}$-dispersion. As a consequence, the latter is strongest towards $\Gamma=(\bar{M}, \pi / c)$ and weakest towards $Z=(\bar{M}, 0)$, where the $z / x y$ band eventually bends over and becomes part of the longitudinal $t / p$ band dispersing upwards from $\mathrm{Z}$ (see bottom panels 
of Fig. 12). This diagonal interlayer coupling (7) thus modulates the long axis, $2 k_{F}$, of the super-ellisoidal cross section such that it becomes minimal towards $\Gamma$ and maximal towards $Z$ (see Fig. 10). Specifically, for the long axis of the $\overline{\mathrm{Y}}$ cylinder: $2 k_{F x}\left(k_{z}\right) \approx 2 k_{F}+\delta k_{F} \cos c k_{z}$, and the same for the long axis of the $\overline{\mathrm{X}}$ cylinder, $2 k_{F y}\left(k_{z}\right)$. Taking then the coupling of the $\overline{\mathrm{X}}$ and $\overline{\mathrm{Y}}$ cylinders into account, we first translate the $\overline{\mathrm{X}}$ cylinder to the $\overline{\mathrm{Y}}$ site and upwards by $\pi / c$, and then couple the two cylinders by the matrix elements (8). The coupling has no effect in the $k_{z}=n \pi / c$ planes containing the $\Gamma, \mathrm{X}$, and Z points, but in the $k_{z}=\pi / 2 c$ planes containing the $\mathrm{P}$ and $\mathrm{N}$ points, the two cross sections mix to become identical around $\mathrm{P}$, as can be seen in the last two top panels of Fig.12. As a result, the double cylinder twists and follows the shape of the string of X-centered rhombic BZ faces (Fig. 2), i.e. it stretches out towards the $z z / z$-bulge around Z of the longitudinal hole sheet. Finally we should mention that the other part of the electron double cylinder is made up of the $\overline{\mathrm{X}} x z / y$ and $\overline{\mathrm{Y}} y z / x$ bands which have no $z$ character and no $k_{z}$-dispersion.

In the two last two top panels of Fig. 12, we observe a Dirac point at $\mathrm{P}$ and $-1.4 \mathrm{eV}$. Its upper cone in this $k_{z}=\pi / 2 c$ plane stays intact over an energy range of nearly $1.5 \mathrm{eV}$ and over a distance of almost $\pi(v=0.5 \mathrm{eV} \cdot a)$ whereafter it develops into the 4 neighboring maxima of the mixed $\overline{\mathrm{M}} t / p$-longitudinal and $\bar{\Gamma} z z / z$ hole band. The lower cone cone extends merely over $0.2 \mathrm{eV}$. In addition, the Dirac point has a second, upper cone which slopes by as much as $v=1.3 \mathrm{eV} \cdot a$ and extends several $\mathrm{eV}$ above the Fermi level, but is truncated slightly below. This is the $x y / z$ electron band. From the cross sections of the band structure with the planes shifted by multiples of $\pi / 4 c$ in Fig.12 one can see that the two upper Dirac cones at the $\mathrm{P}$ points are fairly 3D. This seems to differ from the previously discussed Dirac point at $0.25 \mathrm{eV}$ and $\left(\overline{\mathrm{M}}, k_{\mathrm{D} z}\right)$ whose cones merely extend in a particular $k_{z}=k_{\mathrm{D} z} 2 \mathrm{D}$ plane. In that case, the mechanism is that $k_{z}$ tunes the relative position of two bands, which have different symmetries at a $2 \mathrm{D}$ high-symmetry point, $\overline{\mathbf{k}}_{\mathrm{D}}$, and a hybridization increasing linearly with the distance from that point, to be degenerate at $\mathbf{k}=\left(\overline{\mathbf{k}}_{\mathrm{D}}, k_{\mathrm{D} z}\right)$. Referring to expression (3): $k_{z}$ tunes the gap, which vanishes at the Dirac point, $g\left(k_{z}=k_{\mathrm{D} z}\right)=0$. For the low-energy $z / z z-t / p$ cone at $0.25 \mathrm{eV}$ and $(\overline{\mathrm{M}}, 3 \pi / 4 c), g\left(k_{z}\right)$ is the $k_{z}$ dispersion of the upper $\overline{\mathrm{M}} z / x y-\bar{\Gamma} z z / z$ band seen in Fig. 11 which is larger than that of the cone, $v=0.4 \mathrm{eV} \cdot a$. For the Dirac cones at $\mathrm{P}$, the mechanism is actually the same, but the $k_{z}$ dispersions of the two relevant $z$-like levels are merely from $-1.8 \mathrm{eV}$ and $-1.4 \mathrm{eV}$ at $\mathrm{P}$ to $-1.6 \mathrm{eV}$ and $-1.2 \mathrm{eV}$ at $\mathrm{X}$ (Fig.12), and this amounts to less than the Dirac slopes, $v=0.5$ and $1.3 \mathrm{eV} \cdot a$.

The reason for the small $k_{z}$-dispersion along XPX, compared with that along $\Gamma Z$, is simply that XPX is at the zone boundary where interlayer coupling is between the degenerate $\overline{\mathrm{X}}$ and $\overline{\mathrm{Y}}$ states. Hence, to first order in $t^{\perp}$, the two degenerate $\alpha$ levels split by $\pm t^{\perp}\left|c_{z, \alpha}(\overline{\mathrm{X}})\right|^{2}$, which is independent of $k_{z}$. But if $t^{\perp}$ were as large as $2 \mathrm{eV}$, first order might not suffice. Therefore, once again, we first consult the caption to Fig. 2 to learn that at $\overline{\mathrm{X}}, z$ may only mix with one other state, $y z$, which is the bottom of the $\overline{\mathrm{M}}$ longitudinal $t / p$ band. The interlayer coupling of $\overline{\mathrm{X}}$ with $\overline{\mathrm{Y}}$ therefore requires merely the solution of a $4 \times 4$ matrix, which after exact Löwdin downfolding of the $d$ block reduces to:

$$
\varepsilon\left(\overline{\mathrm{X}}, k_{z}\right)=\epsilon_{z}(\overline{\mathrm{X}})+\frac{t_{z d}^{2}(\overline{\mathrm{X}})}{\varepsilon\left(\overline{\mathrm{X}}, k_{z}\right)-\epsilon_{d}(\overline{\mathrm{X}})} \pm t^{\perp}
$$

These two second-order equations for $\varepsilon\left(\overline{\mathrm{X}}, k_{z}\right)$ can be solved exactly and give no $k_{z}$-dependence. The above-mentioned $0.2 \mathrm{eV} k_{z}$-dependence seen in Fig.12 must therefore be due to interlayer hopping via orbitals other than As $z$, but this is negligible. Secondly, we confirm from Fig. 7 that at $\overline{\mathrm{X}}$, there is essentially only one level with $z$ character (because $t_{z, y z}(\overline{\mathrm{X}})=0.1 \mathrm{eV}$ ). This level is at $-2.1 \mathrm{eV}$ and is non-bonding between nearest neighbors separated by $\mathbf{x} \pm \eta \mathbf{z}$ or $\mathbf{y} \pm \eta \mathbf{z}$. The $y z$ level is at $-1.3 \mathrm{eV}$ and is the one seen in Fig. 12 for $\mathrm{BaFe}_{2} \mathrm{As}_{2}$ to be at $-1.4 \mathrm{eV}$ at $\mathrm{P}$ and $-1.2 \mathrm{eV}$ at $\mathrm{X}$. So if $\mathrm{BaFe}_{2} \mathrm{As}_{2}$ were merely 2D LaOFeAs with added bct interlayer coupling, the shift from -2.1 to $-1.6 \mathrm{eV}$ should be $\sim\left|t^{\perp}\right|$, but that is too inaccurate. In order to find $t^{\perp}$, we therefore seek the lower $z$-level along XPX in $\mathrm{BaFe}_{2} \mathrm{As}_{2}$ and find that it is dispersionless and lies $2.0 \mathrm{eV}$ below the upper $z$ level (and below the frame of the figure). Hence $t^{\perp}=-1.2 \mathrm{eV}$, assuming a $20 \%$ bymixing of $y z$ character to the upper $z$-like level due to the level separation of merely $0.4 \mathrm{eV}$ in $\mathrm{BaFe}_{2} \mathrm{As}_{2}$. 

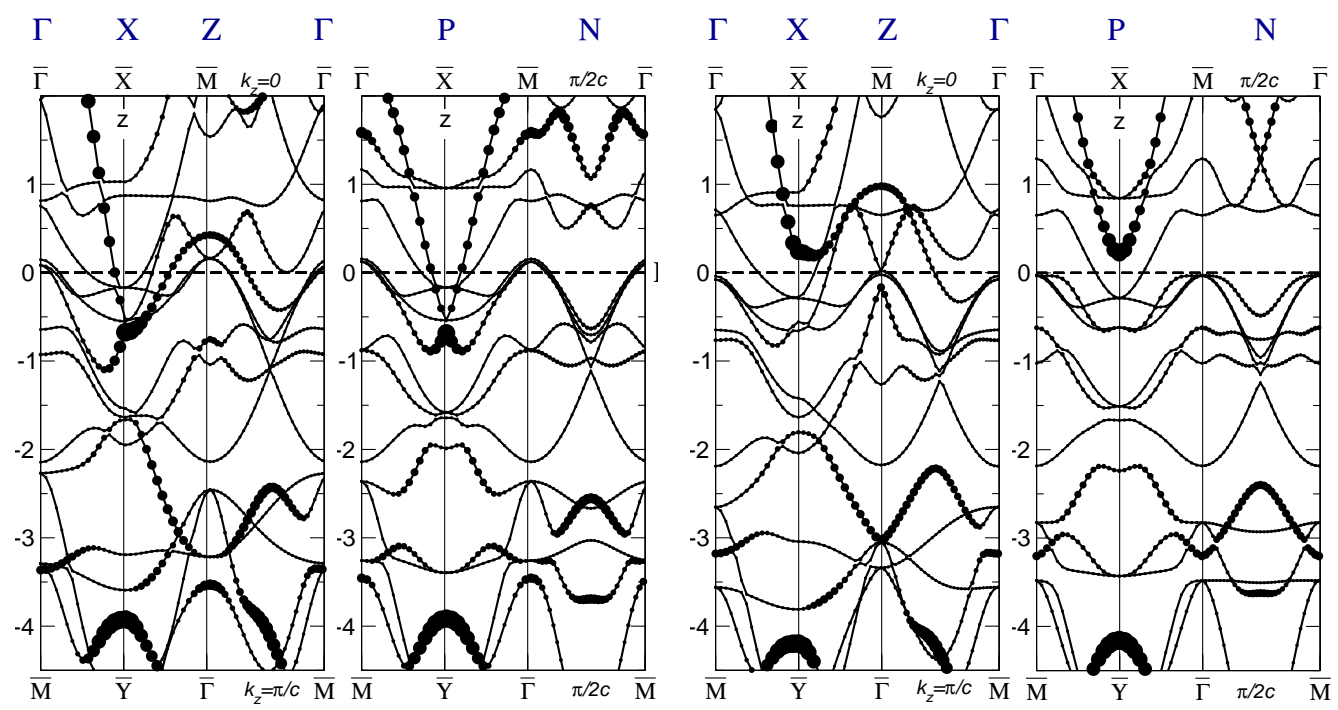

Fig. $133 \mathrm{D}$ band structure of bct $\mathrm{CaFe}_{2} \mathrm{As}_{2}$, fattened by the As $z$ character and along the paths $(\bar{\Gamma} \overline{\mathrm{X}} \overline{\mathrm{M}} \bar{\Gamma}, 0)=$ $(\overline{\mathrm{M}} \overline{\mathrm{Y}} \overline{\mathrm{\Gamma}} \overline{\mathrm{M}}, \pi / c)$ and $(\bar{\Gamma} \overline{\mathrm{X}} \overline{\mathrm{M}} \bar{\Gamma}, \pi / 2 c)=(\overline{\mathrm{M}} \overline{\mathrm{Y}} \bar{\Gamma} \overline{\mathrm{M}}, \pi / 2 c)$. First two panels: normal pressure. Last two panels: Collapsed phase at $0.48 \mathrm{GPa}$. [82] Otherwise as Fig. 12

We remark that although the bands do not disperse along XPX, the wave-function characters of course do, e.g. in the $k_{z}=n \pi / c$ planes, the upper and lower $z$-like levels have respectively purely $\overline{\mathrm{X}} z / y z$ and $\overline{\mathrm{Y}}$ $z / x z$ characters, while in the $k_{z}=\pi / 2 c$ plane, they are completely mixed. Secondly, we remind that the $z$ states along XPX are intra-layer non-bonding and interlayer $p p \sigma$ bonding and antibonding.

Going away from the XPX line, the upper $z$-like band hybridizes linearly with the nearby $z z / z$ and $z / x y$ bands and thereby form the P-centered Dirac cones discussed above. That the cones are centered at the $k_{z}=\pi / 2 c$ plane, i.e. the one containing the high-symmetry points $\mathrm{N}$ and $\mathrm{P}$ (see Fig. 10 ), is due to the fact that, in this plane, the bct bands are periodic for $\overline{\mathbf{k}}$ in the small BZ, whereby the bands along $\bar{\Gamma} \overline{\mathrm{X}}$ equal those along $\bar{M} \bar{Y}$, which by tetragonality equal those along $\bar{M} \bar{X}$. This higher symmetry is clearly seen in the last two top panels of Fig 4

In $\mathrm{CaFe}_{2} \mathrm{As}_{2}, \eta=1.04$ so that the intra-layer $z / x y$ and $z / z z$ hybridizations are smaller than in $\mathrm{Ba}$, whereby the centers of the antibonding $z / x y$ and $z / z z$ bands lie lower. However, of greater importance is that the smaller size of the Ca ion makes the As-As interlayer distance $70 \mathrm{pm}-$ i.e. nearly $20 \%-$ shorter than in in $\mathrm{BaFe}_{2} \mathrm{As}_{2}$. This substantially increases the interlayer hopping $t^{\perp}$. As a result, the splitting of the $z$-band at $\mathrm{X}, \sim 2 t^{\perp}$, which was $2 \mathrm{eV}$ for $\mathrm{Ba}$, is $3.2 \mathrm{eV}$ for Ca. As seen in the first two panels of Fig. 13 . this splitting is from -3.9 to $-0.7 \mathrm{eV}$. Hence, the upper $z$-level is above the $\overline{\mathrm{X}} y z$ and $z z / z$ levels at $\mathrm{X}$ and merely $0.1 \mathrm{eV}$ below the $x y$-like level at $-0.6 \mathrm{eV}$. The consequence is that in $\mathrm{Ca}$, the $x y$-like electron band forms a complete, upper Dirac cone in the $k_{z}=\pi / 2 c$ plane. This cone slopes by about $3.5 \mathrm{eV}$ over the distance $\pi(v=1.1 \mathrm{eV} \cdot a)$ and is thereby twice as steep as the $Y z / X Y$ cone considered after Eq. (3). Contrary to the case in $\mathrm{BaFe}_{2} \mathrm{As}_{2}$, the $x y$-like band now forms the inner electron cylinder.

The second consequence of the upper $z$-level at X lying as high as $-0.7 \mathrm{eV}$, is that the $\bar{\Gamma} z / z z$ hole band lies higher than in $\mathrm{Ba}$. At $\mathrm{Z}$, this amounts to $0.4 \mathrm{eV}$ and places the $\bar{\Gamma} z / z z$ level $0.2 \mathrm{eV}$ above the degenerate $\overline{\mathrm{M}} t / p$ level. Going out in top face of the central BZ from Z, this $\bar{\Gamma} z / z z$ hole band now crosses the longitudinal $t / p$ electron band which, like in $\mathrm{BaFe}_{2} \mathrm{As}_{2}$, curves upwards due to repulsion from the $\overline{\mathrm{M}} z / x y$ band (hybridized with $\mathrm{Ca} 4 d_{z z}$ ). This high-lying $\bar{\Gamma} z / z z$ hole band crosses the Fermi level far outside the transverse $\bar{M} t / p$ hole band. For $k_{z}$ decreasing below $\pi / c$, the crossing between the $\bar{\Gamma} z / z z$ hole band and the $\overline{\mathrm{M}} z / x y$-hybridized longitudinal $\overline{\mathrm{M}} t / p$ electron band gap proportional to sin $c k_{z}$. The resulting lowest band is thus shaped like a volcano with a wide foot of $\bar{\Gamma} z / z z$ character and a caldera of $\bar{M}$ 
$t / p z / x y$ character around $\mathrm{Z}$ and $0.5 \mathrm{eV}$ above the Fermi level. As $k_{z}$ decreases towards $3 \pi / 4 c$, this gap increases so much that the rim is washed out and the caldera develops into a flat hilltop. Eventually, the characters of the $z$-like bands (Fig. 11) gapped around the degenerate $\overline{\mathrm{M}} t / p$ band along $\mathrm{Z} \Gamma$ change back to normal order with the upper band being $\mathrm{M} z / x y$ like and the lower band $\bar{\Gamma} z / z z$ like. As a consequence, the flat hill continuously transforms into the inner, longitudinal $\overline{\mathrm{M}} t / p$ sheet. This can be seen for $k_{z}=\pi / 2 c$ in the second panel of Fig.13. Since the two $z$-like bands are gapped around the $\overline{\mathrm{M}} t / p$ band along $\mathrm{Z} \Gamma$, there is no Dirac point along $\Gamma Z$. The corresponding sheet of Fermi surface is thus a warped, $\Gamma Z$-centered cylinder with a very broad, $\bar{\Gamma} z / z z$-like base near $\mathrm{Z}$ and a long narrow piece around $\Gamma$. Outside of this, except near Z, lies the transversal $\bar{M} t / p$ hole cylinder. The top of the $\bar{\Gamma} x y$ hole band is slightly above that of the $\overline{\mathrm{M}} t / p$ band and its straight cylindrical FS sheet lies outside the transversal $\overline{\mathrm{M}} t / p$ hole cylinder.

Pure $\mathrm{CaFe}_{2} \mathrm{As}_{2}$ becomes superconducting with $T_{c \max } \sim 12 \mathrm{~K}$ without doping but by the application of hydrostatic pressure in the range $2-9 \mathrm{kbar}$. [83] At $5.5 \mathrm{kbar}$ there is a first-order phase transition into a collapsed bet non-magnetic and possibly superconducting phase [84] with $\eta$ marginally smaller and with the interlayer As-As distance decreased by an additional $27 \mathrm{pm}$. In this collapsed phase, whose bands are shown in the two last panels of Fig. 13, the interlayer hopping is increased so much that the splitting of the $z$-band at $\mathrm{X}, \sim 2 t^{\perp}$, is now $4.5 \mathrm{eV}$. As a result, the upper level is $0.2 \mathrm{eV}$ above the Fermi energy. This, first of all means that the electron cylinder has lost one sheet, essentially the $x y / z$ sheet, so that there is no Dirac cone at $\mathrm{P}$. On the other hand, at $\mathrm{Z}$, the $\bar{\Gamma} z / z z$ level is now $1 \mathrm{eV}$ above the Fermi level and the $\overline{\mathrm{M}} z / x y$ level is $0.1 \mathrm{eV}$ below the doubly-degenerate $\overline{\mathrm{M}} t / p$ level. The latter creates a prounced, slightly gapped Dirac cone with $v=0.8 \mathrm{eV} \cdot a$. Moreover, the doubly degenerate $t / p$ level is essentially $a t$ the Fermi level. As $k_{z}$ decreases below $\pi / c$, the $\bar{\Gamma} z / z z$ hole band and the upper Dirac cone, which has mixed longitudinal $t / p$ and $z / x y$ character, gap at their crossing, which is at $0.7 \mathrm{eV}$. This volcano thus has a cone-shaped caldera. As $k_{z}$ decreases towards $\pi / 2 c$, the rim and the Dirac caldera are flattened away and this flat hilltop sinks below the Fermi level. The holes are thus in a large $\bar{\Gamma} z / z z \overline{\mathrm{M}} x y / z$ like sheet shaped as a disc centered at Z. At this center, there may be a non-occupied pin-hole with Dirac character. There are no $\bar{\Gamma} x y$ holes because that band is slightly below $\varepsilon_{F}$. As mentioned above, the electrons are in an XP-centered cylinder of $x z / y z$ character. This band structure is thus very different from the standard one, but quite interesting.

The band structure of $\mathrm{BaRu}_{2} \mathrm{As}_{2}$ [85] is similar to that of (non-collapsed) $\mathrm{CaFe}_{2} \mathrm{As}_{2}$ shown in the first two panels of Fig $[13$, including a Dirac point at P. But it differs in two respects: The $\bar{\Gamma} x y$ hole band is entirely below the Fermi level and the doubly degenerate top of the hole bands disperses like in $\mathrm{BaFe}_{2} \mathrm{As}_{2}$ due to hybridization with $\mathrm{Ba} 5 d_{x z / y z}$ near $\Gamma$. This causes the top of the $t / p$ bands to sink below the Fermi level near $\Gamma$ and the corresponding inner and outer Fermi-surface sheets to truncate.

\section{2D Spin-spiral band structure}

At low temperature and normal pressure, the parent compounds of the Fe-based superconductors (except LiFeAs) become orthorhombic, antiferromagnetic metals. Superconductivity seems to appear, once these spin and charge orders are suppressed, e.g. by doping (electron or hole) or pressure. This superconductivity is presumably mediated by spin-fluctuations.

In this section we shall study the interplay between the band structure and the spin order. Ab-initio calculations employing spin-density-functional theory (SDFT) tend to yield the proper spin order, which is striped with spins on the iron rows along $\mathbf{x}$ aligned and along $\mathbf{y}$ alternating. The moments, albeit still considerably smaller than the saturation magnetization of $4 \mu_{B} / \mathrm{Fe}$, are much larger $\left(\sim 2 \mu_{B} / \mathrm{Fe}\right)$ than those obtained by neutron scattering or muon spin rotation; the latter are $\sim 0.4 \mu_{B} / \mathrm{Fe}$ for LaOFeAs [12] and twice as large for $\mathrm{BaFe}_{2} \mathrm{As}_{2}$. [4] Even worse, only with the calculated large moments do the spin-densityfunctional calculations yield the correct structure $(\eta=0.93$ and $0.5 \%$ contraction in the ferromagnetic direction for $\mathrm{LaOFeAs}$ ); the structures calculated without allowing for spin-polarization differ much more from the observed ones than is normal for density-functional calculations $(\eta=0.81$ and no orthorhombicity for $\mathrm{LaOFeAs}$ ). [38, 63] It thus seems that the large moments exist, but fluctuate on a time scale shorter than what can be resolved with neutrons or muons [72]. Below, we shall first study how the spin-polarization 
modifies the band structures discussed in the previous section which were calculated for the experimental structures. Thereafter we shall consider the energetics of the spin spirals.

\subsection{Formalism}

SDFT involving $d$-electron spins reduces approximately to a Stoner model [86, 87]. This reduction has the conceptual advantage of cutting the SDFT self-consistency loop into a band-structure part which for a given site and orbital-dependent exchange splitting, $\Delta$, yields the site and orbital-dependent spin-moment, $m(\Delta)$, plus a self-consistency condition which simply states that $\Delta=m I$, where $I$ is the Stoner $(\sim$ Hund's rule) interaction parameter. The band-structure part gives insight into the spin response of the non-interacting system, and not only in the linear regime.

The spin arrangements which we shall consider are simple spin spirals. For these, the moment lies in the $(x, y)$-plane and has a constant magnitude, but rotates from site to site by an angle, $\varphi(\mathbf{t})=\mathbf{q} \cdot \mathbf{t}$, proportional to the projection of the lattice translation, $\mathbf{t}$ in Eq. (1), onto the spin-spiral wave vector, $\mathbf{q}$. Hence, the spin spiral with $\mathbf{q}$ at $\bar{\Gamma}$ produces FM order and the one with $\mathbf{q}$ at $\overline{\mathrm{Y}}$ produces stripe order because the moment rotates by $\pi$ upon $\mathbf{y}$-translation, and by 0 upon $\mathrm{x}$-translation. Finally, the spin spiral with $\mathbf{q}$ at $\bar{M}$ produces checkerboard order because the moment rotates by $\pi$ upon $\mathbf{y}$ as well as upon $\mathbf{x}$ translation. These spin spirals with $\mathbf{q}$ at high-symmetry points are collinear and commensurate but with the formalism which we now explain any $\mathbf{q}$ can be treated.

In order to solve the band-structure problem in the presence of such a spin spiral, we use a basis set of localized Wannier orbitals times pure spin-functions, $|\uparrow\rangle$ and $|\downarrow\rangle$, with quantization direction chosen along the local direction of the moment. In this representation, the one-electron Hamiltonian is translationally invariant, albeit with q-dependent hopping integrals, so that there is no coupling between Bloch sums with different wave vectors. As a consequence, the band-structure problem can be solved for any $\mathbf{q}$ without increasing the size of the primitive cell.[88] When merely seeking insights in this section, we shall neglect the interlayer coupling and use the 2D bands in the large BZ. So in this case, configuration space is invariant to the t-mirror group, and spin space is invariant to the t-spinrotation group, which both have the same irreducible representations. As long as spin and orbital spaces remain uncoupled (spin-orbit coupling neglected), the one-electron Hamiltonian therefore factorizes down to the orbital and spin degrees of freedom for a primitive cell of the t-group. This, together with SDFT, enables simple calculation of spin-spiral band structures, moments, and magnetic energies.

The Hamiltonian turns out to be simply:

$$
\tilde{H}_{\mathbf{q}}(\mathbf{k})=\left(\begin{array}{cc}
-\frac{1}{2} \Delta+\frac{1}{2}[h(\mathbf{k})+h(\mathbf{k}+\mathbf{q})] & -\frac{1}{2}[h(\mathbf{k})-h(\mathbf{k}+\mathbf{q})] \\
h . c . & \frac{1}{2} \Delta+\frac{1}{2}[h(\mathbf{k})+h(\mathbf{k}+\mathbf{q})]
\end{array}\right),
$$

in the local $(\uparrow, \downarrow)$ representation and with the origin of $\mathbf{k}$ shifted to $\mathbf{q} / 2$. If the two paramagnetic Hamiltonians, $h(\mathbf{k})$ and $h(\mathbf{k}+\mathbf{q})$, are identical (not merely their eigenvalues), this form is block diagonal. This is also the form appropriate for $\Delta$ larger than the bandwidths. For small $\Delta$, it is more practical to transform to the $(\uparrow \mp \downarrow) / \sqrt{2}$ representation in which

$$
H_{\mathbf{q}}(\mathbf{k})=\left(\begin{array}{cc}
h(\mathbf{k}) & \frac{1}{2} \Delta \\
\frac{1}{2} \Delta & h(\mathbf{k}+\mathbf{q})
\end{array}\right)
$$

In these expressions, $h(\mathbf{k})$ is the paramagnetic $8 \times 8 p d$ Hamiltonian whose eigenvalues, $\varepsilon_{\alpha}(\mathbf{k})$, are the 2D bands discussed in the previous section, and $\Delta$ is an $8 \times 8$ diagonal matrix whose diagonal elements have the same value, $\Delta$, for all five Fe $d$ orbitals, and 0 for all three As $p$ orbitals. The approximation that only like orbitals couple goes back to the assumption that the spin density on Fe is spherically symmetric, and it is justified by the fact that, using this simple form for $\Delta$, we find good agreement with the results of full calculations using SDFT for the spin-polarized bands. Of course, a form with the proper point symmetry on Fe could be used, but that would require more parameters.

Although it takes the spin-spiral representation to see that the Hamiltonian above is general, we do note that, for an $\uparrow$-electron in a commensurate antiferromagnet, a Hamiltonian of the form (12) is obtained 
by elementary means using the $(\Uparrow, \Downarrow)$-sublattice representation, global spin directions, and purely spatial Bloch functions. The Hamiltonian obtained for a $\downarrow$-electron is the same, but with $\Uparrow$ and $\Downarrow$ interchanged.

Diagonalization of the $16 \times 16$ Hamiltonian 13 yields energy bands, $\varepsilon_{\beta}(\mathbf{k})$, and corresponding eigenvectors, $\left\{c_{(\uparrow-\downarrow) l, \beta}(\mathbf{k}), c_{(\uparrow+\downarrow) l, \beta}(\mathbf{k})\right\} \equiv\left\{c_{l, \beta}(\mathbf{k}), c_{l, \beta}(\mathbf{k}+\mathbf{q})\right\}$, with $l$ enumerating the 8 orbitals. (Here, $c_{l, \beta}(\mathbf{k})$ is a simplified notation for one of the 16 eigenvector components; for small $\Delta$, this equals one of the 8 eigenvector components of the paramagnetic Hamiltonian, times $1 / \sqrt{2}$ ). We can now find the orbital-projected spin polarization of state $\beta \mathbf{k}$ as:

$$
p_{l, \beta}(\mathbf{k})=2 \Re c_{l, \beta}^{*}(\mathbf{k}) c_{l, \beta}(\mathbf{k}+\mathbf{q}),
$$

and summing this over the Fe $d$ orbitals, $p_{\beta}(\mathbf{k})=\sum_{l=1,5} p_{l, \beta}(\mathbf{k})$, and over the occupied -or emptystates, we obtain the Fe moment:

$$
m(\Delta)=\sum_{\beta \mathbf{k}}^{\mathrm{occ}} p_{\beta}(\mathbf{k})=-\sum_{\beta \mathbf{k}}^{\mathrm{empty}} p_{\beta}(\mathbf{k}) .
$$

This is the magnetic output of the spin-spiral band-structure calculation.

\subsection{Stripe 2D band structure}

In order to demonstrate how the spin-spiral formalism works for the 2D band structure of LaOFeAs, we start from the paramagnetic bands, $\varepsilon_{\alpha}(\mathbf{k})$, decorated with the weight of each of the eight Wannier orbitals in Fig. 7 We consider a $\bar{Y}$ stripe, and thus prepare for the $\Delta$-coupling as shown in the upper half of Fig. 14: On top of the bands at $\mathbf{k}$ (in green) we place those at $\mathbf{k}+\pi \mathbf{y}$ (in grey). Specifically, on top of the green $\bar{\Gamma} \overline{\mathrm{X}}$ bands we place the grey $\overline{\mathrm{Y}} \overline{\mathrm{M}}$ bands (which are the same as the $\overline{\mathrm{X}} \overline{\mathrm{M}}$ bands with $x z$ and $y z$ exchanged), on top of the green $\overline{\mathrm{X}} \overline{\mathrm{M}}$ bands we place the grey $\overline{\mathrm{M}} \overline{\mathrm{X}}$ bands, and on top of the green $\bar{\Gamma} \overline{\mathrm{Y}}$ bands (which equal the $\bar{\Gamma} \overline{\mathrm{X}}$ bands with $x z$ and $y z$ exchanged) we place the grey $\overline{\mathrm{Y}} \bar{\Gamma}$ band. The $\overline{\mathrm{M}} \bar{\Gamma}$ bands couple with the $\bar{X} \bar{Y}$ bands, but since the latter were not shown in Fig. 7 this line is not shown in Fig. 14 either.

Now, the effect of $\Delta$ is to split degeneracies, $\varepsilon_{\alpha}(\mathbf{k})=\varepsilon_{\beta}(\mathbf{k}+\mathbf{q})$, by $\Delta$ times the geometrical average of the $d$ characters, $\sum_{l=1,5} c_{l, \alpha}^{*}(\mathbf{k}) c_{l, \beta}(\mathbf{k}+\mathbf{q})$. This of course only holds as long as $\Delta$ is so small that no further bands get involved. States without common $d$-character therefore do not split. We note that states throughout the band structure split, independent of the position of the Fermi level, i.e. of the doping, but for small $\Delta$ only those states which gap around the Fermi level contribute to the magnetization and the magnetic energy, so this is how doping enters.

The paramagnetic bands are seen to be linear inside an energy window of $\pm 0.1 \mathrm{eV}$, at the most, around the Fermi level, and this means that effects of the exchange potential $\pm \frac{1}{2} \Delta$ can be treated with linearresponse theory only when $\Delta \lesssim 0.2 \mathrm{eV}$.

\subsubsection{Bands and Fermi surface in the linear-response region, $\Delta \lesssim 0.2 \mathrm{eV}$}

A close inspection of the top $x y$ panel of Fig. 14 reveals that the crossing of the purely $x y$-like band with itself halfway between $\bar{\Gamma}$ and $\bar{Y}$ is $0.3 \mathrm{eV}$ below the undoped Fermi level and thus requires $\Delta>0.6 \mathrm{eV}$ to gap around $\varepsilon_{F}$. On the other hand, the crossing of the $x y$ hole band along $\bar{\Gamma} \bar{Y}$ with the $y z / x$ electron band along $\bar{Y} \bar{\Gamma}$ occurs only slightly below $\varepsilon_{F}$, meaning that the $\bar{\Gamma}$-centered hole pocket and the $\bar{Y}$-centered electron superellipse almost nest along $\bar{\Gamma} \bar{Y}$. This can be seen in Fig. 15 where we show the $\bar{Y}$-folded Fermi surfaces in brown lines. However, the $y z / x$ band has only very weak $x y$ character caused by its weak hybridization with the below-lying $x y$ band, as was explained in connection with Eq. (2). Hence, due to lack of common orbital characters, these two states gap by much less that $\Delta$. Finally, the $x y$ hole band along $\bar{\Gamma} \overline{\mathrm{X}}$ crosses the $x y / z$ electron band along $\overline{\mathrm{Y}} \overline{\mathrm{M}}$ at $\sim 0.1 \mathrm{eV}$ below $\varepsilon_{F}$, but due to the reduced $x y$ character of the $x y / z$ band, $\Delta$ must exceed $\sim 0.3 \mathrm{eV}$ to gap that part of the Fermi surface. As a result, for the value $\Delta=0.18 \mathrm{eV}$ which via Eq. (15) produces the same moment as the one observed experimentally, $m(0.18 \mathrm{eV})=0.3 \mu_{B} / \mathrm{Fe}$, the $x y$ hole pocket does not gap. The Fermi surface calculated for $\Delta=0.18 \mathrm{eV}$ is shown by black lines in Fig.15, For the $\bar{\Gamma}$ and $\bar{Y}$-centered sheets, it only differs from the one calculated 

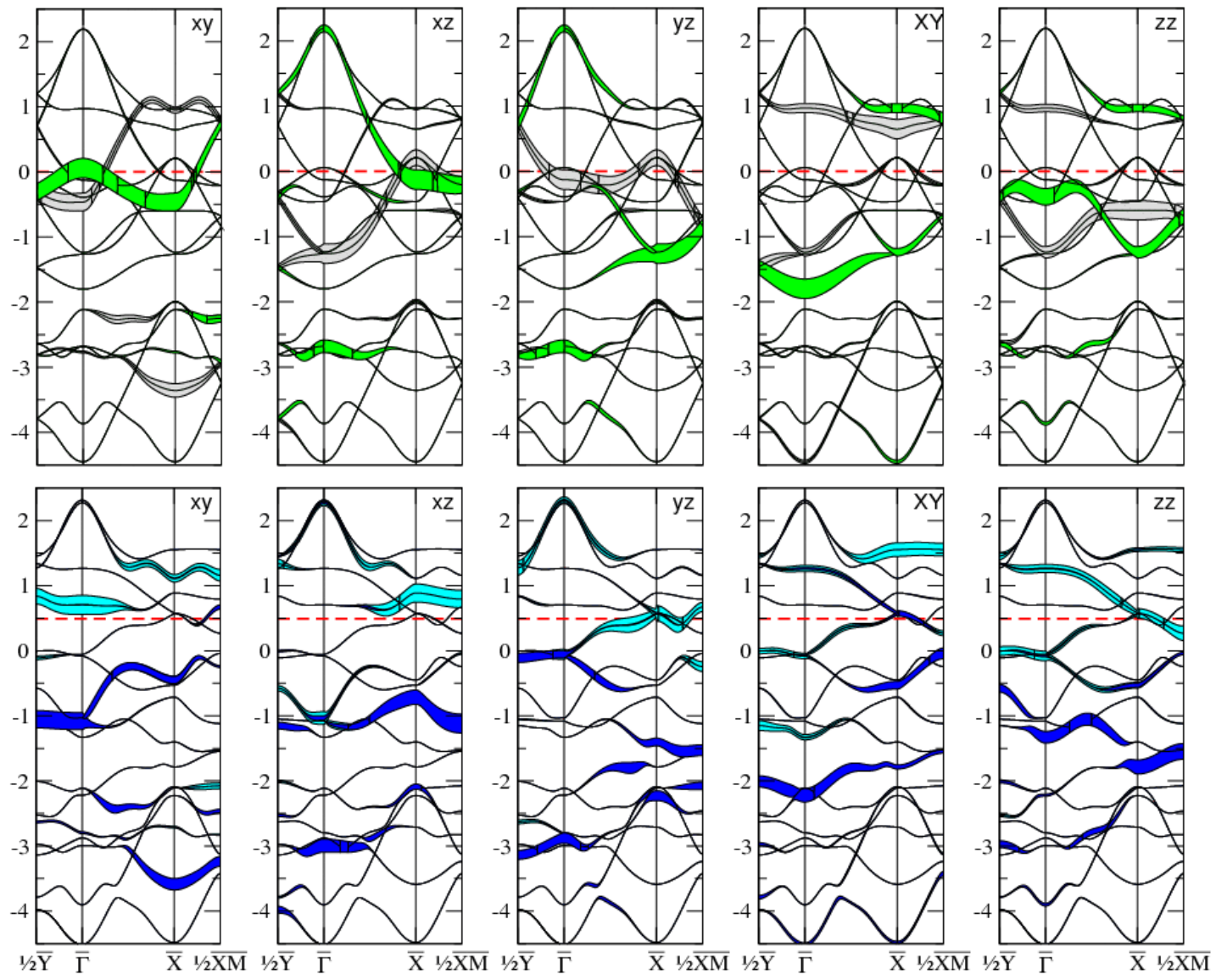

Fig. 14 Top: 2D paramagnetic bands decorated like in Fig. 7 and prepared (folded) for stripe order with $\mathbf{q}=\pi \mathbf{y}$ : On top of the $\bar{\Gamma} \bar{Y}$ bands in green we place the $\bar{Y} \bar{\Gamma}$ bands in grey, on top of the $\bar{\Gamma} \overline{\mathrm{X}}$ bands in green we place the $\bar{Y} \bar{M}$ bands in grey, and on top of the $\bar{X} \bar{M}$ bands in green we place the $\bar{M} \bar{X}$ bands in grey. Bottom: 2D stripe band structure decorated with the orbital-projected spin-polarizations as given by Eq. 14 and with positive and negative polarizations in respectively dark and light blue. For the exchange potential, the value $\Delta=1.8 \mathrm{eV}$ was used, which by Eq. 15 yields the moment $m(1.8 \mathrm{eV})=2.2 \mu_{B} / \mathrm{Fe}$ and corrresponds to the value $I=0.82 \mathrm{eV}$ of the SDFT Stoner parameter. The dashed line is the Fermi level, which has moved up by $0.5 \mathrm{eV}$. Note that the paramagnetic and spin-spiral band structures are lined up with respect to the common paramagnetic potential, i.e. $h(\mathbf{k})$ in the TB Stoner calculation. The 2D stripe Fermi surface is shown in Fig.16 For stripe order, As $p$ projections cannot be spin-polarized and have therefore been omitted.

for $\Delta=0$ and shown in brown lines, because the Fermi level is slightly shifted due to gapping of the other sheets, i.e. those centered at $\overline{\mathrm{M}}$ and $\overline{\mathrm{X}}$. That gapping, which causes the small moment of $0.3 \mu_{B} / \mathrm{Fe}$, takes place as follows:

The side of the $\overline{\mathrm{X}}$-centered electron superellipse which is normal to the $x$ direction, i.e. which points towards $\bar{\Gamma}$, matches the inner, longitudinal $\bar{M}$-centered hole pocket both in Fermi-surface dimension (nesting) and in orbital character, $x z$. Those two bands therefore gap around the Fermi level, while the outer, transversal $(y z)$ hole sheet stays intact. Near the $X$ and $Y$ directions, the $\overline{\mathrm{X}}$ electron sheet however matches the outer, transversal hole sheet in size and orbital character, $Y z$ and $X z$, respectively. So near those directions, the outer, transversal hole sheet is gapped while the inner, longitudinal sheet is intact. Finally, due to lack of common characters near the $y$ direction, where the $x y / z$ electron band does not hybridize 


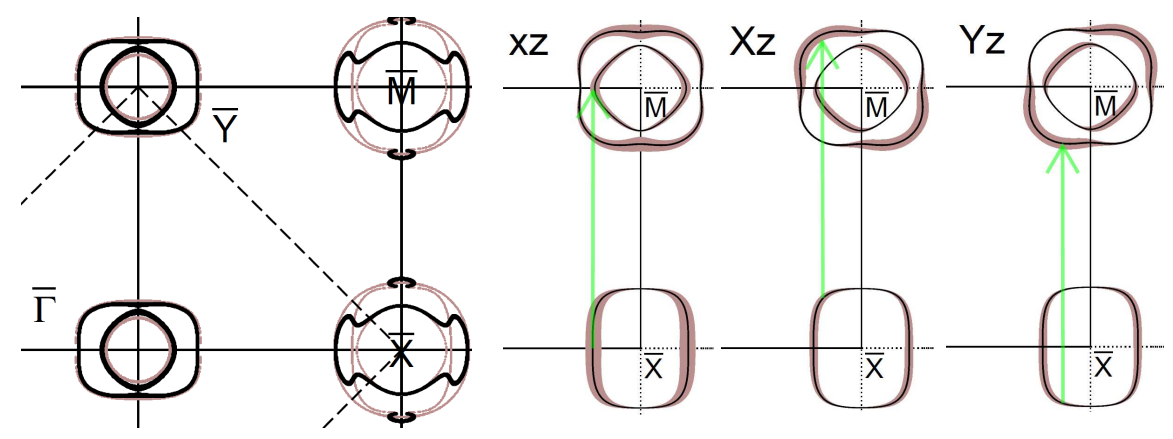

Fig. 15 Right: Nesting of orbital-projected Fermi-surface sheets for undoped 2D LaOFeAs (from Fig. 8) for stripe order with $\mathbf{q}=\pi \mathbf{y}$ (green arrow). Left: Partly gapped Fermi surface resulting from the small exchange potential $\Delta=0.18 \mathrm{eV}$ which yields the small moment $m=0.31 \mu_{B} / \mathrm{Fe}$. This corresponds to the Stoner parameter $I=0.59 \mathrm{eV}$.

with the lower-lying $x z$ band, no gapping occurs. As a consequence, small paramagnetic electron pockets with $x y / z$ and transversal, $x z / X Y / z z / x$ characters occur. Such electron pockets will remain at the Fermi level, also for large $\Delta$, as we shall see below. Note that the FS parts not gapped away are essentially not spin-polarized; this will not be the case for larger $\Delta$. Since the $\bar{Y}$ stripe has antiferromagnetic order in the $y$ direction and ferromagnetic order in the $x$ direction one might expect higher conductivity in the $x$ than in the $y$ direction. However, most of the FS has a predominantly $y$-directed group velocity and predominantly $y z$-electron or $y z$ longitudinal-hole character. Where the velocity is in the $x$ direction, the character is $x y / z$ electron or $y z$ transverse hole. The $x y$ hole pocket is isotropic in the plane. In conclusion, the exchange potential needed $(\Delta=0.18 \mathrm{eV})$ to give the observed moment with the Stoner model, is smaller than the fine structure of the bands. The gapping of the Fermi surface and the susceptibility, $m(\Delta) / \Delta$, therefore depend crucially on the details of the k-and-orbital nesting.

\subsubsection{Bands and Fermi surface beyond the linear-response region, $0.2 \mathrm{eV} \lesssim \Delta<3 \mathrm{eV}$}

The exchange potential obtained selfconsistently from the SDFT and yielding the proper crystal structure, is ten times larger: $\Delta=1.8 \mathrm{eV}$, and thereby has the same scale as the structure of the subbands, i.e. this $\Delta$ is intermediate and linear-response theory invalid. The SDFT value of the Stoner parameter is $I=0.82 \mathrm{eV}$ and the moment is $m(1.8 \mathrm{eV})=2.2 \mu_{B} / \mathrm{Fe}$. Compared with the maximum moment of $4 \mu_{B} / \mathrm{Fe}$ for the $d^{6}$ configuration, the value $2.2 \mu_{B} / \mathrm{Fe}$ is intermediate. The lower part of Fig. 14 now shows the stripe bands for this situation. These bands are complicated, because the gapping and spin-polarization depend on the energies and the $p$ and $d$ orbital characters of those bands at $\mathbf{k}$ and $\mathbf{k}+\mathbf{q}$ which are separated by less than $\sim \Delta$. In the present section, we shall describe those bands and their Fermi surface and calculate specific, important levels analytically.

In Fig. 14 the paramagnetic and the spin-spiral band structures are lined up with respect to the common paramagnetic potential, i.e. $h(\mathbf{k})$ in the TB Stoner calculation. We see, as was pointed out before, that bands with $d$ character split irrespective of their position relatively to the Fermi level, but those in the lower half the $d$-band structure generally shift downwards (dark blue) while those in the upper generally half shift upwards (light blue). We recall that the shift upon an increase of the exchange potential is the negative of the spin-polarization: $\partial \varepsilon_{\beta}(\mathbf{k}) / \partial(\Delta / 2)=-p_{\beta}(\mathbf{k})$, by 1st-order perturbation theory. In fact, there happens to be a fairly well-defined dividing line between positively and negatively spin-polarized bands around $0 \mathrm{eV}$. Moreover, on this dividing line, the non-hybridizing yz and $z z / X Y$ bands along $\bar{\Gamma} \bar{Y}$ are nearly degenerate and dispersionless, so increasing $\Delta$ beyond $1.8 \mathrm{eV}$ will open up a gap which extends throughout the $\mathrm{BZ}$ and makes any correponding $d^{5}$ material (e.g. LaOMnAs or LaOFeN [89]) an antiferromagnetic insulator. 

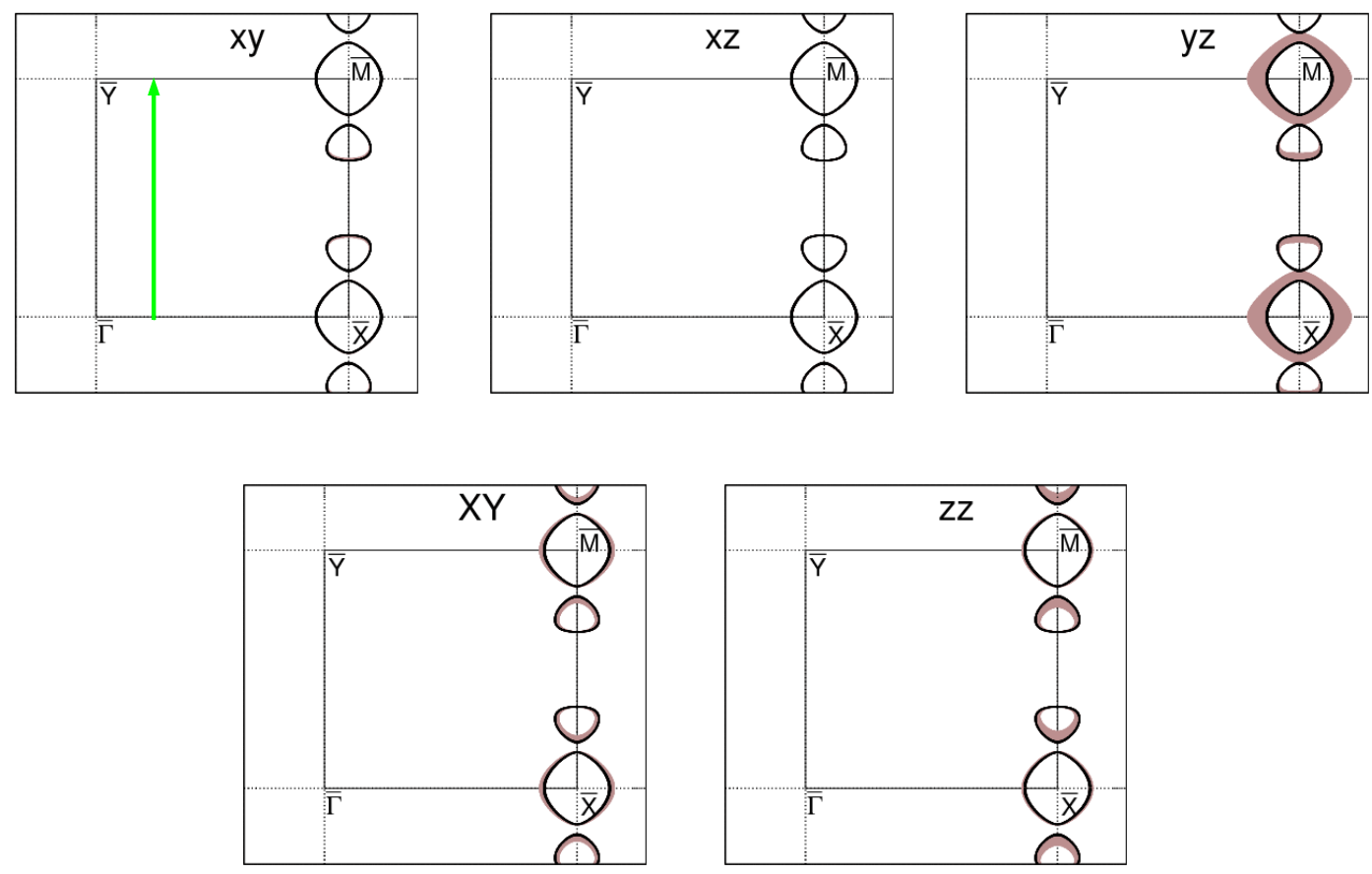

Fig. 16 Fermi surface for stripe order $(\mathbf{q}=\pi \mathbf{y})$ resulting from the large exchange potential $\Delta=1.8 \mathrm{eV}$ which yields the large moment $m=2.2 \mu_{B} /$ Fe corresponding to to the SDFT Stoner value $I=0.82 \mathrm{eV}$. See also Fig.s 15 and 8

We now discuss the intermediate-moment stripe bands for 2D LaOFeAs in detail. Starting again on the left-hand side of Fig.14 with the crossing of the paramagnetic, nearly pure $x y$ band with itself, halfway between $\bar{\Gamma}$ and $\overline{\mathrm{Y}}$ at $-0.3 \mathrm{eV}$, we see the bands split to the energies $-0.3 \pm 0.9 \mathrm{eV}=-0.3 \mathrm{eV} \pm \Delta / 2$ around the Fermi level, which has now moved up to $0.5 \mathrm{eV}$, with the lower and upper bands fully spinpolarized. This gap extends in a large region around the $\bar{\Gamma} \bar{Y}$-line. So, whereas for small $\Delta$, the $\bar{\Gamma} x y$ hole and $\bar{Y} x y$-yz electron sheets did not gap at all, for intermediate $\Delta$, these two FS sheets no longer exist. It is however only the $x y$-parts which are gapped away: Due to strong $y z / x$ hybridization, the $y z$-like bands near $\bar{\Gamma}$ (green) has no partner at $\bar{Y}$ (grey) within the $\pm \Delta / 2$-range with which it can couple. This band therefore only splits midways between $\bar{\Gamma}$ and $\bar{Y}$, i.e. near $\frac{1}{2} \bar{Y}$, but hardly closer to $\bar{\Gamma}$ and towards $\bar{X}$ (grey). Hence, the reason for the disappearance of the $y z$-part of the superellipse at $\bar{Y}$ is not gapping, but the 0.5 $\mathrm{eV}$ upwards shift of the Fermi level. This shift is due to the fact that with configuration $d^{6}$, the Fermi level lies in the upper half of the $d$-like band where most bands are shifted upwards by the exchange potential and drag the Fermi level along with them. The shift $\partial \varepsilon_{F}(\Delta) / \partial(\Delta / 2)$ is upwards, if at $\varepsilon_{F}(\Delta)$ the density of $\downarrow$ states exceeds that of $\uparrow$ states. It may be noted that the crossing of the grey $x y / z$ and $y z$ bands along $\bar{Y} \bar{M}$, which is at $d^{6}$ for the paramagnetic bands, still occurs for the stripe bands, but far below the Fermi energy.

The bands expected to gap mostly for $\bar{Y}$-stripe order are $d$ bands dispersing less than $\Delta / 2$ in the $y$ direction, i.e. the $x z$ and $z z$ bands. The $x z$ band is dispersionless near the $\overline{\mathrm{X}} \overline{\mathrm{M}}$ line where it forms the $\overline{\mathrm{M}}$-centered transverse hole band and the bottom of the $\overline{\mathrm{X}}$-centered electron band. Further towards the $\bar{\Gamma} \overline{\mathrm{Y}}$ line, however, the $k_{y}$-dispersion of the $x z$ band becomes large due to by-mixing of $y$ character towards $\bar{\Gamma}$, 
and also the concomitant dilution of $d$ character reduces the exchange coupling. As a consequence, near the $\overline{\mathrm{X}} \overline{\mathrm{M}}$ line, the $x z$ electron and hole bands split to $\sim \pm \Delta / 2$, whereby the $\downarrow$ band is above the shifted Fermi level and the $\uparrow$ band is far below. This emptying of antibonding $x z$ states yields the observed 0.5 $\%$ orthorhomic contraction in the $x$ direction, along which the spins are aligned ferromagnetically [37]. In the remainder of the zone, there are no bands at the Fermi level. The paramagnetic $z z$ band has a width of about $1 \mathrm{eV} \sim \Delta / 2$ and is centered at $\sim-0.6 \mathrm{eV}$, so we expect the exchange splitting to shift the $z z \downarrow$ band up to -or above- the shifted Fermi level. Fig.14 shows that this is roughly the case, but the details are more complicated, due to strong hybridization with the $X Y$ band, as we shall see later in connection with Eqs.17) and (18). The paramagnetic and stripe bands, albeit merely for positive energies and the exchange splitting, $\Delta=1.1 \mathrm{eV}$, may be seen more clearly in the left-hand side of Fig. 18, We shall return to this figure.

Only two bands remain at the Fermi level: (1) the longitudinal $y z \downarrow$ band dispersing downwards from $\overline{\mathrm{M}}$, crossed by and hybridizing with a weakly spin-polarized $x y / z \uparrow$ band dispersing upwards from $\overline{\mathrm{X}}$, and (2) the $z z \downarrow$ band dispersing downwards from $\bar{M}$, hybridized with more weakly spin-polarized $X Y \uparrow$ band. Being respectively even and odd by reflection in a vertical mirror containing nearest-neighbor As atoms and the $\mathbf{q}$ vector (see Fig. 2), bands 1 and 2 cannot hybridize and therefore cross at a Dirac point along the $\overline{\mathrm{X}} \overline{\mathrm{M}}$ line. This pins the $d^{6}$-Fermi level and gives rise to a Fermi surface shaped like a propeller, [18] with two electron blades and a hole hub (Fig. 16). The hub is $y z \downarrow$ like and the inner parts of the blades are mixed $z z \downarrow-X Y \uparrow$ like, while the outer edges are mixed $y z \downarrow-x y / z \uparrow$ like. Due to the pinning, the propeller shape is robust, e.g. not sensitive to $\Delta$. Hole doping by a few per cent will bring the Fermi level to the Dirac point and make each electron blade shrink to a point. Upon further hole doping, the blades will reappear as hole sheets. The velocity of the $x y / z \uparrow$ and the $z z \downarrow$ parts of the anisotropic cone are respectively $\sim 1 \mathrm{eV} \cdot a=2.9 \mathrm{eV} \AA$ and $\sim 0.4 \mathrm{eV} \cdot a=1.1 \mathrm{eV} \AA$. These Dirac cones in the stripe-ordered SDW state have been predicted [78] and later observed using respectively quantum oscillations [31] and ARPES [79]. Compared with ours, the experimental velocities seem to be renormalized by a factor $\sim 1 / 4$. This Dirac cone will be gapped by any lattice imperfection breaking the above-mentioned mirror symmetry and is therefore not "protected."

In order to explain how the complicated spin and orbital characters of conduction bands 1 and 2 arise from the paramagnetic band structure, let us consider the simple case that the paramagnetic TB Hamiltonian $h(\mathbf{k})$ in Eq. (13) is a $2 \times 2$ matrix. Its eigenvalues are the bonding, $\varepsilon_{b}(\mathbf{k})$, and antibonding, $\varepsilon_{a}(\mathbf{k})$, paramagnetic bands with the respective eigenvectors, $\{-\sin \phi(\mathbf{k}), \cos \phi(\mathbf{k})\}$ and $\{\cos \phi(\mathbf{k}), \sin \phi(\mathbf{k})\} \equiv$ $\{c(\mathbf{k}), s(\mathbf{k})\}$ (we have chosen the phases of the orbitals such that the Hamiltonian is real). Taking the first orbital is a $d$ and the second as a $p$ orbital, transformation of the spin-spiral Hamiltonian (13) to the bonding-antibonding representation yields:

$$
H_{p d, \mathbf{q}}(\mathbf{k})=\left(\begin{array}{cccc}
\varepsilon_{a}(\mathbf{k}) & 0 & \frac{1}{2} \Delta c(\mathbf{k}) c(\mathbf{k}+\mathbf{q}) & -\frac{1}{2} \Delta c(\mathbf{k}) s(\mathbf{k}+\mathbf{q}) \\
0 & \varepsilon_{b}(\mathbf{k}) & -\frac{1}{2} \Delta s(\mathbf{k}) c(\mathbf{k}+\mathbf{q}) & \frac{1}{2} \Delta s(\mathbf{k}) s(\mathbf{k}+\mathbf{q}) \\
c . c . & c . c . & \varepsilon_{a}(\mathbf{k}+\mathbf{q}) & 0 \\
c . c . & c . c . & 0 & \varepsilon_{b}(\mathbf{k}+\mathbf{q})
\end{array}\right)
$$

because the $p p$ and $p d$ elements of the exchange block, $\frac{1}{2} \Delta$, vanish. Here, $c^{2}$ and $s^{2}=1-c^{2}$ are the $d$ characters of the antibonding and bonding levels, respectively. Note that only the $d$-but not the $p-$ characters need to be the same at $\mathbf{k}$ and $\mathbf{k}+\mathbf{q}$. This $4 \times 4$ form (16) is exact when $\mathbf{k}$, and thereby $\mathbf{k}+\pi \mathbf{y}$, is at a high-symmetry point, because here, $h(\mathbf{k})$ factorizes into blocks of dimension $\leq 2 \times 2$, with the $X Y / z z / x$-block at $\overline{\mathrm{X}}$ as the notable exception (see caption to Fig.2). We thus start explaining the spin and orbital characters of band 1 by using $(16)$ to couple the levels at $\mathbf{k}=\overline{\mathrm{X}}$ to those at $\mathbf{k}+\mathbf{q}=\overline{\mathrm{M}}$ : the paramagnetic $x y$-like antibonding and bonding levels at $\overline{\mathrm{X}}$ (see Figs.7 and 14) are the strongly $x y$-like bottom of the electron band (green), for which $\varepsilon_{a}(\overline{\mathrm{X}})=-0.46 \mathrm{eV}$ and $c(\overline{\mathrm{X}})=0.95$, and the strongly $y$-like level at $\varepsilon_{b}(\overline{\mathrm{X}})=-2.79 \mathrm{eV}$. These levels couple to the $x y$-like levels at $\overline{\mathrm{M}}$ (grey) of which the antibonding one at $\varepsilon_{a}(\overline{\mathrm{M}})=0.95 \mathrm{eV}$ is mostly $z$-like, and the bonding one at $\varepsilon_{b}(\overline{\mathrm{M}})=-3.36 \mathrm{eV}$ is mostly $x y$-like, 
$s(\overline{\mathrm{M}})=0.83$. The four $x y$-like stripe levels at $\overline{\mathrm{X}}$ are thus the eigenvalues of the Hamiltonian:

$$
\left(\begin{array}{cccc}
-0.46 & 0 & (\Delta / 2)(0.95)(0.56) & -(\Delta / 2)(0.95)(0.83) \\
0 & -2.79 & -(\Delta / 2)(0.31)(0.56) & (\Delta / 2)(0.31)(0.83) \\
\text { c.c. } & \text { c.c. } & 0.95 & 0 \\
\text { c.c. } & \text { c.c. } & 0 & -3.36
\end{array}\right)
$$

These eigenvalues (seen in Fig.14 are: $1.12,-0.45,-2.73$, and $-3.59 \mathrm{eV}$ when $\Delta / 2=0.9 \mathrm{eV}$, and hence little perturbed by the stripe order. The reasons are that the paramagnetic levels are separated by more than $\Delta / 2$, and that the $p$ hybridization reduces the geometrical averages of the $d$ characters far beyond unity, except for two levels which are, however, separated by as much as $3 \mathrm{eV}$. In particular the state of interest, the one at $-0.45 \mathrm{eV}$, has been pushed down by the level at 0.95 and up by the one at -3.36 , both at $\overline{\mathrm{M}}$, and as a result, has moved by merely $0.01 \mathrm{eV}$. For the same reason, its spin polarization is only about $50 \%$. This state thus remains essentially the (green) bottom of the electron band at $\overline{\mathrm{X}}$.

The paramagnetic $y z$-like antibonding and bonding levels at $\overline{\mathrm{X}}$ are the strongly $y z$-like bottom of the longitudinal hole band, for which $\varepsilon_{a}(\overline{\mathrm{X}})=-1.26 \mathrm{eV}$ and $c(\overline{\mathrm{X}})=0.998$, and the strongly $z$-like level at $\varepsilon_{b}(\overline{\mathrm{X}})=-2.12 \mathrm{eV}$. These levels couple to the $y z$-like levels at $\overline{\mathrm{M}}$ (grey), which are top of the doubly degenerate hole band, for which $\varepsilon_{a}(\overline{\mathrm{M}})=0.21 \mathrm{eV}$ and $c(\overline{\mathrm{M}})=0.90$, and the $y$-like level at $\varepsilon_{b}(\overline{\mathrm{M}})=-1.97 \mathrm{eV}$. With these values, the $y z$-like eigenvalues of the $4 \times 4$ stripe Hamiltonian (16) becomes $0.58,-1.39,-2.11$, and $-2.21 \mathrm{eV}$. Here the uppermost level, being near the Fermi level, is our band 1. It is described to a good approximation by using merely the antibonding paramagnetic states, i.e. by the $2 \times 2$ Hamiltonian

$$
\left(\begin{array}{cc}
\varepsilon_{a}(\mathbf{k}) & \frac{\Delta}{2} c(\mathbf{k}) c(\mathbf{k}+\mathbf{q}) \\
\frac{\Delta}{2} c(\mathbf{k}) c(\mathbf{k}+\mathbf{q}) & \varepsilon_{a}(\mathbf{k}+\mathbf{q})
\end{array}\right)=\left(\begin{array}{cc}
-1.26 & (\Delta / 2)(0.998)(0.90) \\
c . c . & 0.21
\end{array}\right) \mathrm{eV},
$$

because these states are the only ones with substantial $y z$ character, as may also be seen from Fig. 14, The uppermost state thus has about $80 \% \overline{\mathrm{M}} y z$ and $20 \% \overline{\mathrm{X}} y z$ character, and its spin polarization is $-75 \%$.

As we now move from $\overline{\mathrm{X}}$ towards $\overline{\mathrm{M}}$, via $\frac{1}{2} \overline{\mathrm{X}} \overline{\mathrm{M}}$ as in Figs. 14 and 18 , the $\overline{\mathrm{X}} x y / z \uparrow$ band disperses upwards from $-0.45 \mathrm{eV}$ like the paramagnetic (green) electron band, and the $y z \downarrow$ band disperses downwards from $0.58 \mathrm{eV}$ like the paramagnetic (grey) longitudinal $\overline{\mathrm{M}} y z$ hole band. At about $1 / 4$ the distance to $\overline{\mathrm{M}}$, these two bands suffer an avoided crossing. From there on, the $x y / z \uparrow$ band continues towards $\bar{M}$ like the paramagnetic band, apart from the facts (a) that it gets folded and split at $\frac{1}{2} \overline{\mathrm{X}} \overline{\mathrm{M}}$ with a covalency-reduced $\Delta$, and (b) that it continues to hybridize with the longitudinal $y z \downarrow$ band whose downwards dispersion (grey) is halted near $\frac{1}{2} \overline{\mathrm{X}} \overline{\mathrm{M}}$ due to repulsion from the upcoming (green) $y z$ band. The $x y / y z$ hybridization matrix element along $\bar{X} \bar{M},-2 \sqrt{2}\left(t_{x y, X z}^{10}+t_{x y, X z}^{11}+t_{x y, X z}^{21}\right) \sin k_{y}$, would have vanished, if the $x y$ and $X z$ Wannier orbitals of the $p d$ set shown in Fig. 6had been respectively symmetric and antisymmetric with respect to the Fe plane. Band 1 obtained from the $p d$ Stoner model is somewhat more shallow than the one obtained from a standard LAPW calculation, which yields a bandwidth of $0.6 \mathrm{eV}$. This can be traced back to the gap between the downwards-dispersing longitudinal $y z \downarrow$ band and the upwards dispersing $x y / z \uparrow$ band, being $0.5 \mathrm{eV}$ in the model but merely $0.2 \mathrm{eV}$ in the LAPW calculation, thus causing the model band to be $0.3 \mathrm{eV}$ more narrow. Reducing $t_{x y, X z}^{10}+t_{x y, X z}^{11}+t_{x y, X z}^{21}$ does not entirely remove this discrepancy, which might also be due to our assumption of a spherically symmetric exchange potential.

We now come to band 2. It was pointed out in Sect. 3.3, and can clearly be seen in Figs. 7 and 14 that the paramagnetic $X Y$ and $z z$ bands hybridize strongly, except along the $\bar{\Gamma} \bar{M}$ lines, and have avoided crossings around $\bar{X}$ and $\bar{Y}$ causing them to gap around the Fermi level. They also gap around the Fermi level along $\bar{\Gamma} \bar{M}$, but due to avoided crossings with other bands. In order to understand the effect of a stripe potential, let us use a $4 \times 4$ model like (16), but now for two $d$ orbitals. In this case, the exchange block is constant and the spin-spiral Hamiltonian in the bonding-antibonding representation becomes:

$$
H_{d d, \mathbf{q}}(\mathbf{k})=\left(\begin{array}{cccc}
\varepsilon_{a}(\mathbf{k}) & 0 & \frac{1}{2} \Delta \cos (\phi(\mathbf{k}+\mathbf{q})-\phi(\mathbf{k})) & -\frac{1}{2} \Delta \sin (\phi(\mathbf{k}+\mathbf{q})-\phi(\mathbf{k})) \\
0 & \varepsilon_{b}(\mathbf{k}) & \frac{1}{2} \Delta \sin (\phi(\mathbf{k}+\mathbf{q})-\phi(\mathbf{k})) & \frac{1}{2} \Delta \cos (\phi(\mathbf{k}+\mathbf{q})-\phi(\mathbf{k})) \\
c . c . & c . c . & \varepsilon_{a}(\mathbf{k}+\mathbf{q}) & 0 \\
c . c . & c . c . & 0 & \varepsilon_{b}(\mathbf{k}+\mathbf{q})
\end{array}\right) .
$$


Clearly, if the bonding and antibonding linear combinations of the two $d$ orbitals were the same at $\mathbf{k}$ and $\mathbf{k}+\mathbf{q}$, i.e. if $\phi(\mathbf{k})=\phi(\mathbf{k}+\mathbf{q})$, then the bonding-antibonding representation (17) would be identical with the $d d$ representation. In that representation, the off-diagonal block is $\frac{1}{2} \Delta$ times the unit matrix because we have assumed the exchange potential to be spherical. If now also $\varepsilon_{a}(\mathbf{k})=\varepsilon_{a}(\mathbf{k}+\mathbf{q})$ and $\varepsilon_{b}(\mathbf{k})=\varepsilon_{b}(\mathbf{k}+\mathbf{q})$, as would be the case at the zone boundary, then we could transform to the local spin representation (12) and would then immediately realize that the 4 stripe eigenvalues (in $\mathrm{eV}$ ) are:

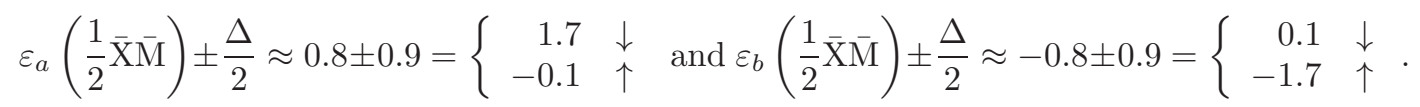

This fits well with Fig.14, from where we have taken the values 0.8 and $-0.8 \mathrm{eV}$, for the paramagnetic antibonding and bonding levels at $\frac{1}{2} \overline{\mathrm{X}} \overline{\mathrm{M}}$. The stripe level belonging to band 2 is the bonding, minority-spin level at $0.1 \mathrm{eV}$. To be honest, the energies $\pm 0.8 \mathrm{eV}$ of the paramagnetic levels do include weak hybridizations with the $x z$ and $x$ orbitals, which have been neglected in the $4 \times 4(X Y, z z)$ model (17). We should also warn that although $h(\mathbf{k})$ and $h(\mathbf{k}+\mathbf{q})$ have the same eigenvalues at the zone boundary, these matrices are generally not identical; off-diagonal elements may have different signs, e.g. $h_{X Y, z z}\left(-\frac{\pi}{2} \mathbf{y}\right)=h_{X Y, z z}\left(\frac{\pi}{2} \mathbf{y}\right)$ but $h_{x z, z z}\left(-\frac{\pi}{2} \mathbf{y}\right)=-h_{x z, z z}\left(\frac{\pi}{2} \mathbf{y}\right)$.

As seen from the figures, the paramagnetic bonding and antibonding $X Y / z z$ bands disperse less than their separation, $\delta \sim 1.6 \mathrm{eV}$, so in order to be able to diagonalize the $4 \times 4$ stripe Hamitonian (17) let us stay with the assumption that $\varepsilon_{a}(\mathbf{k})=\varepsilon_{a}(\mathbf{k}+\mathbf{q}) \equiv \delta / 2$ and $\varepsilon_{b}(\mathbf{k})=\varepsilon_{b}(\mathbf{k}+\mathbf{q}) \equiv-\delta / 2$, but drop the assumption that $\phi(\mathbf{k})=\phi(\mathbf{k}+\mathbf{q})$. The 4 stripe bands are then given by:

$$
\varepsilon(\mathbf{k})= \pm \frac{1}{2} \sqrt{\delta^{2} \pm 2 \delta \Delta \cos \varphi(\mathbf{k})+\Delta^{2}}, \quad \text { where } \quad \varphi(\mathbf{k}) \equiv \phi(\mathbf{k}+\mathbf{q})-\phi(\mathbf{k}) .
$$

For $\varphi=0$, the zone-boundary case (18), the bonding and antibonding $d$ orbitals are identical and each of the four levels, $\pm(\delta / 2) \pm(\Delta / 2)$, have pure spin and pure bonding or antibonding character. For $\varphi=\pi / 2$, the bonding and antibonding bands have orthogonal $d$ characters and therefore only have off-diagonal exchange coupling. This does not split the spin-degenerate bonding and antibonding levels, but separates them to $\pm \frac{1}{2} \sqrt{\delta^{2}+\Delta^{2}}$. The realistic case at $\overline{\mathrm{X}}$ (green) is that the antibonding and bonding levels have roughly the same $X Y$ and $z z$ characters. We therefore take $\phi(\overline{\mathrm{X}})=\pi / 4$, and the $X Y$ orbital as the first orbital. At $\bar{M}$ (grey) the "antibonding" level has pure $X Y$ and the "bonding" level pure $z z$ character, so $\phi(\bar{M})=0$. As a result, the bands have dispersed from the levels given by (18) to $\pm \frac{1}{2} \sqrt{\delta^{2} \pm \sqrt{2} \delta \Delta+\Delta^{2}}=$ \pm 1.57 and $\pm 0.66 \mathrm{eV}$ at $\overline{\mathrm{X}}=\overline{\mathrm{M}}$. This agrees well with what is seen in Fig.14 and explains why band 2 disperses upwards from 0.1 at $\frac{1}{2} \overline{\mathrm{X}} \overline{\mathrm{M}}$ to $0.66 \mathrm{eV}$ eV at $\overline{\mathrm{X}}$. The latter level is essentially bonding $z z \downarrow$ $/ X Y \uparrow$.

Armed with the detailed understanding of the interlayer hopping provided in Sect. 4 and that of the generic 2D stripe band structure provided above, the interested reader should be able to digest the complicated 3D stripe bands for specific materials found in the literature and recently reviewed in Refs. [3, 4].

\subsection{Magnetization and magnetic energy}

Apart from the spin-spiral band structure described above in the case of stripe order, the output of a bandstructure calculation with an imposed exchange potential, $\Delta$, is the Fe-magnetization, $m(\Delta)$. In Fig. 17 we now give the results obtained for stripe $(\mathbf{q}=\pi \mathbf{y})$ and checkerboard $(\mathbf{q}=\pi \mathbf{x}+\pi \mathbf{y})$ orders for various electron dopings, $\mathrm{x}$, in the rigid-band approximation. With the Stoner approximation, we have been able to afford sampling the spin-polarization over a very fine $k$-mesh so that nesting features are resolved. Since the magnetization increases linearly with $\Delta$, when it is small, we plot the static spin suceptibility, $\chi(m) \equiv m(\Delta) / \Delta$, and as a function of $m$ rather than of $\Delta$, since the $m$ is an observable. Note that with 

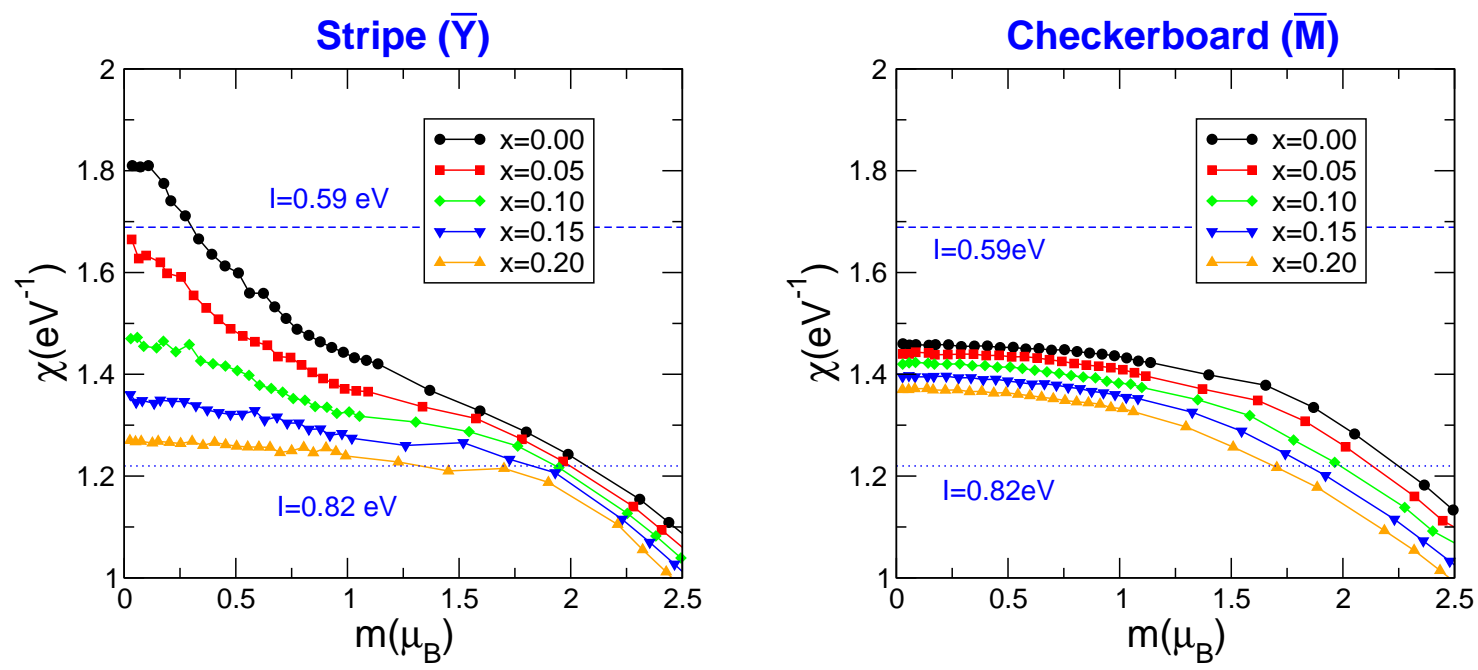

Fig. 17 Non-interacting, static spin susceptibilities $\chi(m) \equiv m / \Delta$ for 2D LaOFeAs calculated from spin-spiral band-structure calculations, i.e. from diagonalizing $H_{\mathbf{q}}(\mathbf{k})$ in Eq. 13) and finding $m$ from the eigenvectors according to Eq. 15. The paramagnetic TB $p d$ Hamiltonian, $h(\mathbf{k})$, was calculated for the observed structure. The electron dopings, $\mathrm{x}$ (in e/Fe), were varied in the rigid-band approximation. For a given value of the Stoner interaction parameter, $I$, the self-consistent moment is given by $\chi(m)=1 / I$. The SDFT value of $I$ is $0.82 \mathrm{eV}$ and the value fitting the experimental moment and its doping dependence is $0.59 \mathrm{eV}$.

$4-\mathrm{x}$ empty bands, $4-\mathrm{x}$ is the value of the saturation magnetization and $\chi(m)$ therefore vanishes for $m$ larger than this.

Given a value of the Stoner exchange-coupling constant, $I$, the self-consistent value of the magnetization is the solution of the equation $\chi(m)=1 / I$, and we see that for the SDFT value, $I=0.82 \mathrm{eV}, m \sim 2.2 \mu_{B} / \mathrm{Fe}$ for both stripe and checkerboard order, and that $m$ decreases with electron doping. The reason for the latter can be understood by considering the stripe bands for $\Delta=1.8 \mathrm{eV}$ at the bottom of Fig. 14. The magnetization along the upwards-sloping line $\chi=m /(1.8 \mathrm{eV})$ in Fig. 17 is the sum over the empty bands of their spin polarizations, taken with the opposite sign according to Eq [15), i.e. of the light-blue fatness. Since lightblue is seen to dominate over dark-blue, at every energy above the Fermi level, moving the latter $u p$, as electron doping will do in the rigid-band spproximation, must decrease the moment.

Coming now to the small-moment part of Fig. 17, the linear response, $\chi(0)$, is seen to be particularly large for stripe order and no doping. This is due to the good nesting shown in Fig. 15 between the $x z$ part of the $\overline{\mathrm{X}}$-centered electron superellipse and those of the $\overline{\mathrm{M}}$-centered hole pockets. This nesting is, however, sensitive to the relative sizes of electron and hole sheets and is therefore rapidly destroyed with electron (or hole) doping, thus causing $\chi(0)$ to decrease. Also, increasing $\Delta$ beyond $0.2 / 1.8 \sim 0.1 \mathrm{eV}$ is seen to make $\chi(m)$ decrease rapidly. We should remember (Fig. 4) that the top of the $\bar{\Gamma}$-centered $x y$-like hole pocket is merely $0.06 \mathrm{eV}$ above the Fermi level for the pure material and that this pocket disappears once the doping exceeds $0.1 \mathrm{e} / \mathrm{Fe}$. We recall also, that the top of the $\bar{M}$-centered hole pockets is merely $0.2 \mathrm{eV}$ above the pure Fermi level and that these pockets disappear, as well, once the electron doping exceeds $0.3 \mathrm{e} / \mathrm{Fe}$. The $\bar{\Gamma}$-centered hole pocket and the $x y$-part of the $\bar{Y}$-centered electron superellipse start to gap when $\Delta$ exceeds $0.2 \mathrm{eV}$, and for larger $\Delta$, the $x y$ moment becomes as large as the $x z$ moment. The experimentally observed moment in LaOFeAs is $\sim 0.4 \mu_{B} / \mathrm{Fe}$, stripe ordered, and vanishes for $\mathrm{x} \gtrsim 3 \%$. This would be consistent with our 2D bands and the Stoner model if $I=0.59 \mathrm{eV}$. However, only by virtue of its large moment, $\sim 2 \mu_{B}$, does the SDFT yield the observed large value of the As height, $\eta=0.93$, and the observed $0.5 \%$ orthorhombic contraction in the direction of ferromagnetic order.

For checkerboard order, $\mathbf{q}$ is at the $\bar{M}$ point and this places the $\bar{\Gamma}$ and $\bar{M}$-centered hole sheets -as well as the $\bar{X}$ and $\bar{Y}$-centered electron sheets- on top of each other. Nesting of sheets with the same (electron 

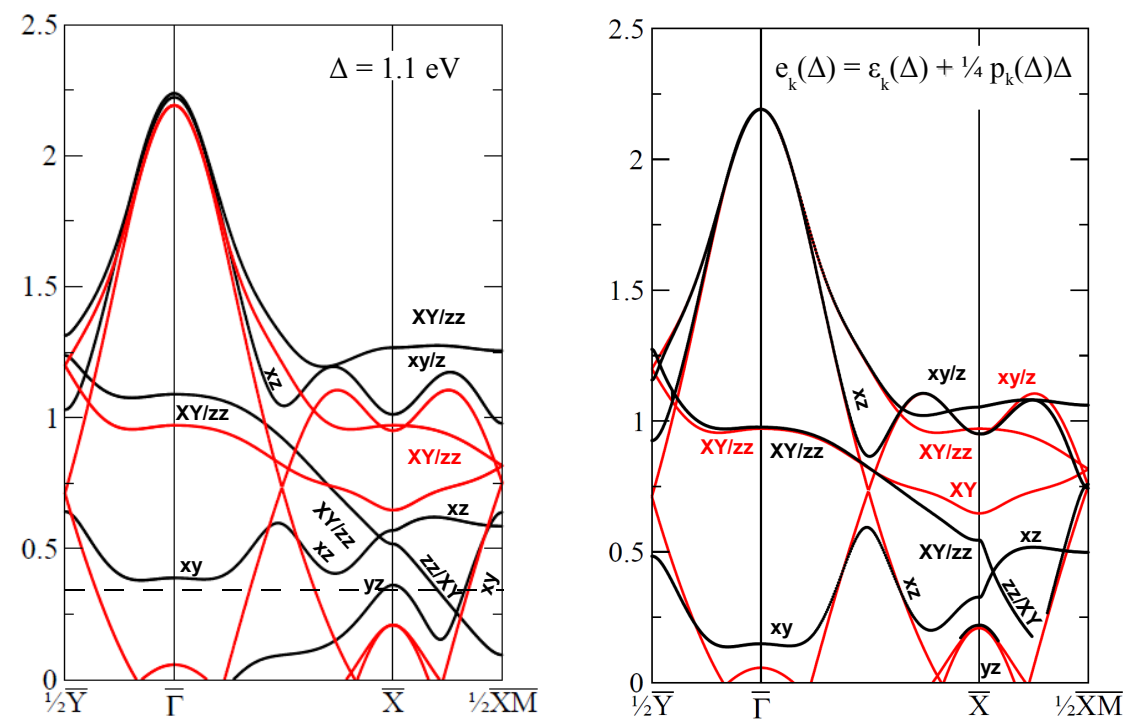

Fig. 18 Band-resolved magnetic energy for stripe order (q at $\bar{Y})$. Red: Unoccupied part of the folded paramagnetic band structure, i.e. $\varepsilon_{\alpha}(\mathbf{k})$ and $\varepsilon_{\alpha}(\mathbf{k}+\mathbf{q})$ for $\Delta=0$. This is the upper part of Fig. 14, but only for positive energies and without fatness. Black left: Band structure, $\varepsilon_{\beta}(\Delta, \mathbf{k})$, obtained by applying an exchange potential of intermediate strength, $\Delta=1.1 \mathrm{eV}$, yielding a magnetization of $1.5 \mu_{B} / \mathrm{Fe}$ and thus corresponding to $I=0.73 \mathrm{eV}$. The Fermi level (dashed line), $\varepsilon_{F}(\Delta)$, has moved up by $0.3 \mathrm{eV}$. The dominant $d$-orbital characters have been written onto the bands. Black right: Unoccupied part of the band structure, $e_{\beta}(\Delta, \mathbf{k}) \equiv \varepsilon_{\beta}(\Delta, \mathbf{k})+\frac{1}{4} p_{\beta}(\Delta, \mathbf{k}) \Delta$, corrected for double counting such that the magnetic energy gain per $\beta \mathbf{k}$-hole is $e_{\beta}(\Delta, \mathbf{k})-\varepsilon_{\beta}(0, \mathbf{k})$, i.e. black minus red. Note that only the unoccupied part of the bands are shown and, in particular, that the double-counting corrected $e$-bands are truncated by the $\varepsilon$-band Fermi level, $\varepsilon_{F}(\Delta)$, a truncation which does not occur at the same energy for all $e$-bands.

or hole) character is not optimal for gapping, and $\chi(0)$ is therefore neither very high nor very doping dependent.

For moments so low that for all possible spin orientations $m(\Delta)$ is linear and the magnetic energy quadratic, the effective coupling between the spins can be expressed in terms of the (Stoner enhanced) linear, static spin susceptibility. For moments so large that the system is insulating, on the other hand, the electronic degrees of freedom can be integrated out, whereby the coupling between the spins is given by a Heisenberg model. That model, with 1 st and 2nd-nearest neighbor antiferro-magnetic couplings and $J_{1} \lesssim 2 J_{2}$ has, in fact, often been used to describe the magnetism of the iron pnictides. This is, however, hardly justified because the iron pnictides are metals, presumably with intermediate moments. Full SDFT calculations, like the spin-spiral calculation described at the end of this section, do however account well for many experimental observations and as a first step towards deriving better exchange models, we shall therefore try to explain the origin of the magnetic energies using the Stoner model.

The relation between the magnetic energy for a particular spin spiral, a $\bar{Y}$ stripe, and the underlying band structure is illustrated in Fig.18. Its left-hand side shows the paramagnetic bands for positive energies, i.e. the unoccupied bands, as well as the stripe bands for the somewhat reduced value $\Delta=1.1 \mathrm{eV}$ of the exchange splitting. This corresponds to $I=0.73 \mathrm{eV}$ and to an SDFT calculation for $\mathrm{BaFe}_{2} \mathrm{As}_{2}$ adjusted to the experimental dHvA FS [61]. The dashed line shows the Fermi level, $\varepsilon_{F}(\Delta)=0.3 \mathrm{eV}$. Note that, like in Fig.14 the paramagnetic and spin-spiral band structures are lined up with respect to the common paramagnetic potential. We clearly see that the $\Delta=1.1 \mathrm{eV}$ stripe perturbs all bands, but the $p$-like ones the least.

In SDFT, the total energy is a stationary functional of the electron and spin densities. For densities which can be generated by occupying the solutions of a single-particle Schrödinger equation for a local 
potential according to Fermi-Dirac statistics, the value of this functional is simply the sum of the occupied single-particle energies, minus corrections for double-counting of the Hartree and exchange-correlation energies. This holds when the potential is the self-consistent one, i.e. the one which minimizes the energy functional. For our Stoner model with $h(\mathbf{k})$ describing the self-consistent paramagnetic bands and for $\Delta$ taking the self-consistent value, $m(\Delta) I$, the double-counting correction of the magnetic energy is simply $\frac{1}{4} m(\Delta) \Delta=\sum_{\beta \mathbf{k}}^{\mathrm{occ}} \frac{1}{4} p_{\beta}(\Delta, \mathbf{k}) \Delta$. For $d^{6}$ materials, we shall sum over empty states, because of those there are only $4 / \mathrm{Fe}$, and nearly all have negative spin polarization. The double-counting correction of the stripe bands has now been performed on the right-hand side of Fig.18 from where we realize that these (black) bands, $e_{\beta}(\Delta, \mathbf{k}) \equiv \varepsilon_{\beta}(\Delta, \mathbf{k})+\frac{1}{4} p_{\beta}(\Delta, \mathbf{k}) \Delta$, are far less perturbed than the real stripe bands, $\varepsilon_{\beta}(\Delta, \mathbf{k})$. This means, that the state-resolved magnetic energy gain (black minus red), $e_{\beta}(\Delta, \mathbf{k})-\varepsilon_{\beta}(0, \mathbf{k})$, is concentrated near the exchange gaps and near the paramagnetic and spin-spiral Fermi surfaces. Note that each of these empty bands, $e_{\beta}(\Delta, \mathbf{k})$ and $\varepsilon_{\beta}(0, \mathbf{k})$, should be defined as $0 \equiv \varepsilon_{F}(0)$, if that band is occupied. This means that the empty paramagnetic bands, $\varepsilon_{\beta}(0, \mathbf{k})$, are continuous, but truncated with a kink at the lower figure frame. The empty, corrected magnetic bands, $e_{\beta}(\Delta, \mathbf{k})$, should be truncated discontinuously. Due to the $0.3 \mathrm{eV}$ upwards shift of $\varepsilon_{F}(\Delta)$, all empty parts of the corrected bands are above the frame of the figure so that all empty magnetic bands are visible.

In order to see which states contribute to the energy of the $\bar{Y}$ stripe, let us now once again start from $\frac{1}{2} \bar{Y}$ and move to the right in the right-hand figure. The zone-boundary gapping around $1.2 \mathrm{eV}$ of the corrected $X Y / z z / x z / y$ band is small and fairly localized near $\frac{1}{2} \bar{Y}$, and there, its positive and negative contributions nearly cancel. The gapping around $0.7 \mathrm{eV}$ of the $y z / x y / x$ band is much larger due to the dominating $y z$ character of this band, but here again, the negative and positive contributions essentially cancel, until $\mathbf{k}$ gets closer to $\bar{\Gamma}$. There, the character of the lower band is $x y$ from the $\bar{\Gamma}$-centered hole band and $x y / z$ from the $\bar{Y}$-centered superellipse electron band. This band contributes positively to the energy of the stripe in the large region of the BZ around $\bar{\Gamma}=\overline{\mathrm{Y}}$ where the FS is completely gapped, a contribution which integrates up to about half the stripe energy. Between $\bar{\Gamma}$ and $\overline{\mathrm{X}}$, the black $x y / z$ band is seen to suffer an avoided crossing with the band formed from the $\overline{\mathrm{X}}$-centered $x z / y$ electron band and the longitudinal $\overline{\mathrm{M}}$-centered $x z$ hole band. Near the avoided crossing the magnetic energy density becomes negative, but is essentially cancelled by the positive contribution from the upper band. Closer to $\overline{\mathrm{X}}$, the contribution from the lower band becomes positive again, and its positive magnetic energy density is seen to extend over the large region around $\overline{\mathrm{X}}=\overline{\mathrm{M}}$ where the $x z$ part of the FS is completely gapped. This part of the band is formed by coupling of the paramagnetic, flat $\overline{\mathrm{X}}$-centered $x z$ electron band, which is occupied and therefore lies below the frame af the figure, and the paramagnetic transversal $\bar{M}$-centered $x z$ hole band, which becomes occupied outside the transversal hole pocket. So whereas the empty, black $x y-x z$ band extends smoothly throughout the BZ, its paramagnetic partner, against which we measure the band-resolved magnetic energy, goes to zero at a few places in the BZ, such as outside the transversal $\overline{\mathrm{M}}$-centered hole band along $\overline{\mathrm{X}} \overline{\mathrm{M}}$. The magnetic one-electron energy of the $x z$-like band thus reaches $+0.5 \mathrm{eV}$ between $\overline{\mathrm{X}}$ and $\frac{1}{2} \overline{\mathrm{X}} \overline{\mathrm{M}}$.

Also the two uppermost black bands, which are degenerate at $\bar{\Gamma}$ and then split into longitudinal and transversal $p / t$ bands, stay empty, i.e. they extend smoothly throughout the BZ. The lower of these bands, the one which is longitudinal $p / t$-like near $\bar{\Gamma}$, becomes $\overline{\mathrm{M}} z / x y$ like near $\overline{\mathrm{X}}$ and is seen to contribute negligibly to the magnetic energy in LaOFeAs (but possibly not in the bct materials). The same holds between $\frac{1}{2} \overline{\mathrm{X}} \overline{\mathrm{M}}$ and $\sim \frac{1}{3} \overline{\mathrm{X}} \overline{\mathrm{M}}$ for its lower partner, which is essentially the paramagnetic $\overline{\mathrm{X}}$-centered $x y / z$ electron band extending in the direction towards $\overline{\mathrm{M}}$. At $\frac{1}{3} \overline{\mathrm{X}} \overline{\mathrm{M}}$, where the magnetic band becomes the tip of the propeller blade, the corrected $x y / z$ band jumbs to 0 , whereby the one-electron magnetic energy jumps from 0 to $-0.25 \mathrm{eV}$. Also the $\overline{\mathrm{M}}$-centered $y z$ hole band coincides with the corrected magnetic $y z$ band, so that only the Fermi-surface truncations contribute to the magnetic energy, which in this case amounts to a small negative energy from the region between the propeller hub and the $y z$-part of the $\bar{M}$-centered hole pockets.

The higher of those two bands which are degenerate at $\bar{\Gamma}$, i.e. the one which is transversal $p / t$ near $\bar{\Gamma}$, develops into the uppermost of the four bands formed by the coupling of the paramagnetic $\overline{\mathrm{X}} X Y / z z$ and $\overline{\mathrm{M}} X Y$ bands described in connection with Eq. [19), albeit for a larger $\Delta$. This band is seen to contribute 

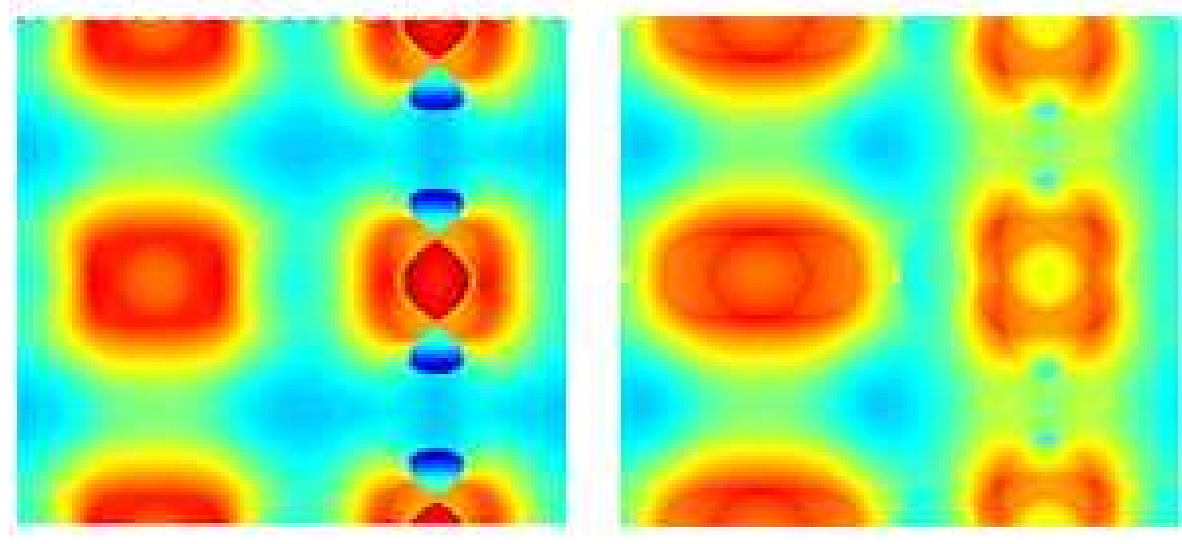

Fig. 19 k-resolved magnetic $\bar{Y}$-stripe energy, $\sum_{\beta}^{\text {empty }}\left[e_{\beta}(\Delta, \mathbf{k})-\varepsilon_{\beta}(0, \mathbf{k})\right]$, as in Fig. 18 but for $\Delta=2.3 \mathrm{eV}$ and summed over the empty bands and shown throughout $\left(k_{x}, k_{y}\right)$-space. Magnetic energy gains are red and losses blue. The discontinuities caused by the propeller sheet are clearly seen. While the left-hand figure is for $2 \mathrm{D}$ LaOFeAs with the observed structure $(\eta=0.93)$, the right-hand figure shows the result obtained by reducing $t_{x y, z}^{\frac{1}{2} \frac{1}{2}}$ from 0.52 to $0.30 \mathrm{eV}$. With the latter value, the 2D paramagnetic $z / x y$ band becomes degenerate with the top of the hole bands at $\overline{\mathrm{M}}$, and thus forms a Dirac cone with the longitudinal $t / p$ band. This corresponds to the elongation $\eta \sim 1.2$.

positively to the magnetic energy, a contribution which is, however, overwhelmed by a large, negative contribution from the second of the four bands, which is part of the $X Y / z z$ band decreasing from $1.3 \mathrm{eV}$ at $\frac{1}{2} \overline{\mathrm{Y}}$ to $0.6 \mathrm{eV}$ at $\overline{\mathrm{X}}$, and finally to $0.2 \mathrm{eV}$ around $\frac{1}{4} \overline{\mathrm{X}} \overline{\mathrm{M}}$. At this point the $z z / X Y$ band is on the inner part of the propeller blade, and therefore jumps discontinuously to 0 , where is stays until reaching the zone boundary at $\frac{1}{2} \overline{\mathrm{X}} \overline{\mathrm{M}}$. The magnetic one-electron energy of the $z z / X Y$-like band thus jumps from -0.6 to $-0.8 \mathrm{eV}$ at $\frac{1}{4} \overline{\mathrm{X}} \overline{\mathrm{M}}$.

It is simpler to eyeball the balance of magnetic one-electron energies along the line between $\overline{\mathrm{X}}$ and $\frac{1}{2} \overline{\mathrm{X}} \overline{\mathrm{M}}$, where we found large cancellations, if we connect the magnetic $X Y / z z$ and $x z$ bands according to energy. In fact, the real $z z / X Y$ and $x z$ bands may cross along $\bar{\Gamma} \overline{\mathrm{X}}$ and $\overline{\mathrm{Y}} \overline{\mathrm{M}}$, but not between $\overline{\mathrm{X}}$ and $\frac{1}{2} \overline{\mathrm{X}} \overline{\mathrm{M}}$. When doing so, we see that the magnetic energy loss from the $X Y / z z-x z$ band lying near $0.5 \mathrm{eV}$ is nearly balanced by the gain provided by the upper $X Y / z z$ band lying near $1 \mathrm{eV}$. The lower $x z-z z / X Y$ band lies $\sim 0.2 \mathrm{eV}$ above the $x z$ part of the paramagnetic $\bar{M}$-centered hole band and thus gains considerable magnetic energy. The Fermi-surface contribution seems to be small because $k_{F y}$ for the outer, transversal $\bar{M}$-centered hole surface is about the same dimension as the distance from the center of the hub to the inner, $z z / X Y$ part of the blade. Finally, by joining this $x z-z z / X Y$ band across the blade to the $x y / z$ band, we see that there is a loss of one-electron magnetic energy of about $0.2 \mathrm{eV}$ inside the blade.

This is seen quite clearly in the left-hand part of Fig. 19 where we show the k-resolved magnetic energy, which is the state-resolved magnetic energy considered above, summed over empty bands. What stabilizes stripe order when its moment is $\gtrsim 1 \mu_{B} / \mathrm{Fe}$, is then, first of all, coupling of the paramagnetic $\bar{\Gamma}$-centered $x y$ hole and the $\overline{\mathrm{Y}}$-centered $x y / z$ electron bands over a large part of $\mathbf{k}$-space centered at $\bar{\Gamma}$. The second, almost as large contribution comes from coupling of the $x z$ part of the paramagnetic $\bar{M}$-centered hole band to that of the $\overline{\mathrm{X}}$-centered electron band over a smaller part of the zone centered at $\overline{\mathrm{X}}$. These $\bar{\Gamma}$ and $\overline{\mathrm{X}}$ centered red regions do not overlap. The Fermi-surface contributions to the magnetic energy are relatively small, and the positive (red) contribution from the hub tends to cancel the negative (blue) contribution from the blades. This being the case, it should be possible to derive a Heisenberg model which fairly accurately describes the change of the magnetic energy for pertubations of $\mathbf{q}$ around commensurable stripe order.

We can now address the interplay between the distance between the As and Fe sheets and the stripe magnetism: The main effect of increasing this distance, $\eta$, is to decrease the $z-x y$ hybridization, as was 

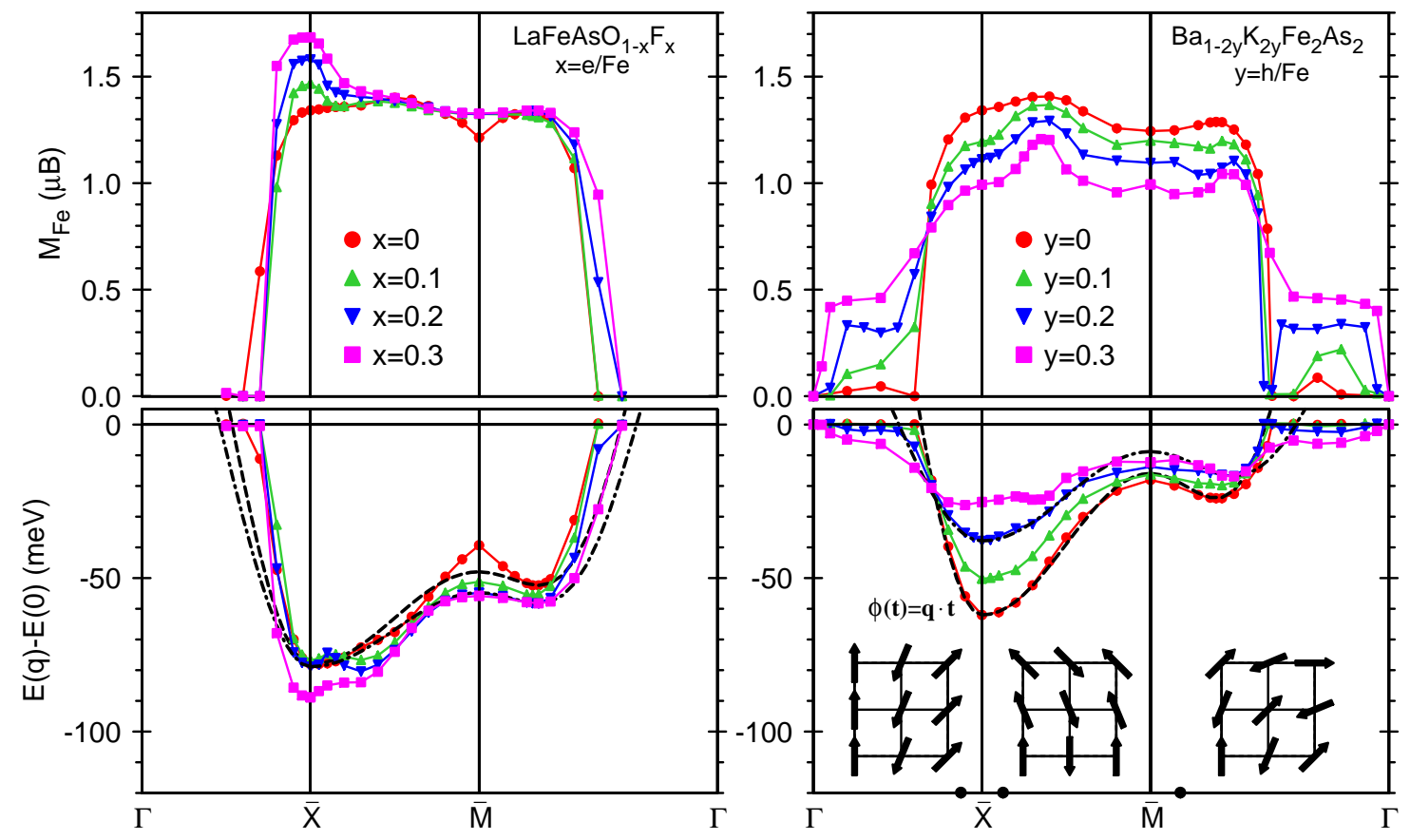

Fig. 20 Magnetic moments (upper panels) and energies (lower panels) per Fe of spin spirals as functions of $\mathbf{q}$ for different electron (x) and hole (y) dopings in the virtual-crystal approximation. These results were obtained by selfconsistent SDFT-LMTO calculations. [48] The energies of the $J_{1}, J_{2}$ Heisenberg model for $\mathrm{x}(\mathrm{y})=0$ and 0.2 are given by respectively dashed and dash-dotted lines. Representative real-space spin structures are shown at the bottom right for the q-vectors denoted by dots (adapted from Ref. [48]).

discussed in Sect. 4.1, and thereby to decrease the splitting between the paramagnetic $z / x y$ antibonding and $x y / z$ bonding levels near $\overline{\mathrm{M}}$, and thus to move the empty $\overline{\mathrm{M}} z / x y$ level down. The $z / x y$ electron band from $\overline{\mathrm{Y}}=\bar{\Gamma}$ to $\overline{\mathrm{M}}=\overline{\mathrm{X}}$, as well as the ones folded from $\overline{\mathrm{X}}$ to $\frac{1}{2} \overline{\mathrm{X}} \overline{\mathrm{M}}$ (see Fig]14), will be less steep and more $x y$-like upon increasing $\eta$. This, in turn, will increase the polarization and the gapping of the $x y$-like stripe bands and thereby increase the fixed- $\Delta$ moment, $m(\Delta)$, and the differential suceptibility, $\chi \equiv d m(\Delta) / d \Delta$. The increase of the self-consistent moment will finally be enhanced over the increase of $m(\Delta)$ by the Stoner factor $(1-I \chi)^{-1}$. At the same time as the moment increases, so does the gapping, the stripe energy, and, hence, the magnetic energy. Also the mere flattening of the $z / x y$ bands increases the magnetic energy by extending the regions around $\bar{\Gamma}$ and $\overline{\mathrm{X}}$ of positive magnetic one-electron energy. As seen in the right-hand part of Fig. 19 , the $\bar{\Gamma}$ region increases in the $k_{x}$ direction and the $\overline{\mathrm{X}}$ region in the $k_{y}$ direction. This explains why spin polarization tends to increase the vertial Fe-As distance.

In conclusion, what stabilizes stripe order when its moment is $\gtrsim 1 \mu_{B} / \mathrm{Fe}$, is -first of all- the coupling of the paramagnetic $\bar{\Gamma}$-centered $x y$ hole and $\overline{\mathrm{Y}}$-centered $x y / z$ electron bands over a large part of $\mathbf{k}$-space centered at $\bar{\Gamma}$. The second, almost as large contribution comes from coupling of the $x z$ part of the paramagnetic $\bar{M}$-centered hole band to that of the $\overline{\mathrm{X}}$-centered electron band over a smaller part of the zone centered at $\overline{\mathrm{X}}$. These $\bar{\Gamma}$ and $\overline{\mathrm{X}}$-centered regions do not overlap. The Fermi-surface contributions to the magnetic energy are comparatively small and mostly negative. This being the case, it should be possible to derive fairly accurate Heisenberg models describing the change of the magnetic energy for pertubations of $\mathbf{q}$ around commensurable stripe order. For a start, one might compute the spin-spiral energy dispersions as a function of $\mathbf{q}$, using the simple TB Stoner model (13) and then analyse which one-electron states are reponsible for the energy changes, like we did for $q=\pi \mathbf{y}$.

The results shown so far were obtained using the spherical Stoner model, which allowed us to simplify the calculation of the spin-spiral band structures and magnetic energies so much, that we might understand 
the results by solving simple analytical problems. Although approximate, this model is in many respects more general than SDFT calculations. Now, coming to the end of our tutorial paper, we show in Fig. 20 results of SDFT spin-spiral calculations for $\mathrm{LaO}_{1-\mathrm{x}} \mathrm{F}_{\mathrm{x}} \mathrm{FeAs}$ and $\mathrm{Ba}_{1-2 \mathrm{y}} \mathrm{K}_{2 \mathrm{y}} \mathrm{Fe}_{2} \mathrm{As}_{2}$ of self-consistent moments and energies as funtions of $\mathbf{q}$ and doping in the virtual-crystal approximation (VCA). [48] Note that in this figure, $\mathbf{q}$ takes the usual $\bar{\Gamma} \overline{\mathrm{X}} \overline{\mathrm{M}} \bar{\Gamma}$ path, so that the spin-spiral patterns near $\overline{\mathrm{X}}$ correspond to an $\overline{\mathrm{X}}$ stripe. For LaOFeAs, the moment is seen to be $\sim 1.3 \mu_{B} / \mathrm{Fe}$ for both stripe and checkerboard order, which is somewhat smaller than what we obtained in Fig.17 presumably because the moment is calculated by integration of the spin-polarization in an Fe sphere with radius $\sim a / 2$ in the SDFT calculation, rather than being summed over $\mathrm{Fe}$ Wannier orbitals. Other causes could be our use of the Stoner aproximation with too high an $I$ and a spherical $\Delta$. More significant is, however, that whereas we found, and understood, that in the rigid-band approximation the large moment decreases with electron doping, and increases with hole doping, the behaviour seems to be the opposite in the VCA approximation, both for electron-doped $\mathrm{LaO}_{1-\mathrm{x}} \mathrm{F}_{\mathrm{x}} \mathrm{Fe}$ As and hole-doped $\mathrm{Ba}_{1-2 \mathrm{y}} \mathrm{K}_{2 \mathrm{y}} \mathrm{Fe}_{2} \mathrm{As}_{2}$. The VCA approximates $\mathrm{O}_{1-\mathrm{x}} \mathrm{F}_{\mathrm{x}}\left(\right.$ or $\left.\mathrm{Ba}_{1-2 \mathrm{y}} \mathrm{K}_{2 \mathrm{y}}\right)$ by a virtual atom having a non-integer number of protons. Such anomalies have recently been discussed for Co and $\mathrm{Ni}$-substitution at the $\mathrm{Fe}$ site using supercell calculations, [90] but not for substitution in the blocking layers. We may speculate that at least for $\mathrm{Ba}_{1-2 \mathrm{y}} \mathrm{K}_{2 \mathrm{y}} \mathrm{Fe}_{2} \mathrm{As}_{2}$, the strong $\mathrm{Ba} 5 d$ hybrididization near the Fermi level found in Sect.4.2 could make substitution of Ba by $\mathrm{K}$ a non-trivial doping. Nevertheless, the spin-spiral energies shown in the lower half of Fig.20 agree with experiments to the extent that the stable spin order is the stripe for low doping, but shifts to in-commensurable order for higher doping, $\mathrm{x}>5 \%$ in LaOFeAs. This is consistent with the onset of superconductivity in this material, but is a completely different, and presumably more accurate scenario than the one suggested by Fig. 17. The black dashed and dotted lines are fits by a simple Heisenberg nearest and next-nearest neighbor $\left(J_{1}, J_{2}\right)$ model to the calculated spin-spiral dispersions for respectively the undoped and $20 \%$ doped compounds. These fits are not bad, although exchange interactions of longer range are needed to fit the LaOFeAs incommensurability. However, the SDFT energies of other spin arrangements, such as starting from stripe order and then rotating the spins on one Fe sublattice rigidly with respect to those on the other, could not be reproduced by the $\left(J_{1}, J_{2}\right)$ model.

\section{Summary}

We hope to have given a pedagogical, self-contained description of the seemingly complicated multiorbital band structures of the new iron pnictide and chalcogenide superconductors. First, we derived a generic Fe $d$ As $p$ TB Hamiltonian by NMTO downfolding of the DFT band structure of LaOFeAs for the observed crystal structure. By use of the glide mirror symmetry of a single FeAs layer, its primitive cell was reduced to one FeAs unit, i.e. the TB $p d$ Hamiltonian, $h(\mathbf{k})$, is an $8 \times 8$ matrix whose converged analytical expressions are, however, too long to for the present paper. We specified how $h(\mathbf{k})$ factorizes at points -and along lines of high symmetry in the $2 \mathrm{D} \mathrm{BZ}$ and pointed to the many band crossings and linear dispersions ("incipient Dirac cones") caused by the factorizations. Their role, together with that of $\mathrm{Fe} d$ As $p$ hybridization for the presence of the $d^{6}$ pseudogap at the Fermi level and the details of the shapes and masses of the electron and hole pockets were subsequently explained. Thereafter we included interlayer coupling, which mainly proceeds via the As $z$ orbitals, and showed how the st and bct 3D band structures can be obtained by coupling $h(\mathbf{k})$ and $h(\mathbf{k}+\pi \mathbf{x}+\pi \mathbf{y})$, i.e. by folding the $2 \mathrm{D}$ bands into the small BZ. This formalism allowed us to explain, for the first time, we believe, the complicated DFT band structures of in particular st $\mathrm{SmOFeAs}$, bct $\mathrm{BaFe}_{2} \mathrm{As}_{2}$, bct $\mathrm{CaFe}_{2} \mathrm{As}_{2}$, and collapsed bct $\mathrm{CaFe}_{2} \mathrm{As}_{2}$. What causes the complications are the material-dependent level inversions taking place as functions of $k_{z}$. We found several Dirac points near the Fermi level. Whether these points have any physical implications remains to be seen. They do not pin the Fermi level, because there are also other FS sheets, and they are not protected, because they are merely caused by crystal symmetries, such as the vertical mirrorplane containing the nearest-neighbor As atoms, which are easily broken by phonons, impurities a.s.o. 
We then studied the generic band structures in the presence of spin spirals, whose Fe moment has a constant value, $m$, but whose orientation spiral along with wavevector $\mathbf{q}$. The formalism simply couples $h(\mathbf{k})$ to $h(\mathbf{k}+\mathbf{q})$ and does not require $\mathbf{q}$ to be commensurate. We used the Stoner approximation to SDFT, because it is simple and allows one to calculate the spin-spiral band structures as functions of the strength, $\Delta$, of the exchange potential and impose the selfconsisteny condition, $\Delta=m(\Delta) I$ at a later stage. We limited ourselves to using this formalism to explain the 2D band structures for stripe order as a function of $\Delta$, but in quite some depth, often using simple analytical theory. What complicates the magnetic band structure is the simultaneous presence of As-Fe covalency and Stoner-exchange coupling. That the latter is only between like $\mathrm{Fe} d$ orbitals, gave the structure of the small-moment SDW-gapping of the paramagnetic FS as well as the intermediate-moment propeller-shaped FS . With the goal of eventually understanding -and possibly simplifying the calculation of- the spin spiral energy dispersions, we expressed the magnetic energy as the difference between magnetic and nonmagnetic band-structure energies, whereby the magnetic band structure should be the one corrected for double counting of the exchange interaction. This formalism was then applied to the large-moment stripe order and we found its stabilization energy to have two main sources (1) the coupling of the paramagnetic $x y$ hole and $x y / z$ electron bands and (2) the coupling of the other electron band and the $x z$ part of the doubly degenerate hole band. The Fermi-surface contributions to the magnetic energy were found to be comparatively small, and that gave some hope for developing a suitable Heisenberg Hamiltonian. We also explained the much discussed coupling in SDFT between the stripe moment and the As height above the Fe plane. In the end, we showed and discussed the selfconsistent spin-spiral moment and energy dispersion obtained from a SDFT calculation, co-authored by one of us. [48]

\section{Acknowledgements}

We would like to thank Alexander Yaresko for pointing out to us the beauty of spin spirals. Dmytro Inosov convinced us about the non-triviality of interlayer coupling in $\mathrm{BaFe}_{2} \mathrm{As}_{2}$. Maciej Zwierzycki provided us with computer programs using the Overlapping-Muffin-Tin-Approximation (OMTA). [91] We are grateful to Claudia Hagemann for proof-reading the manuscript, and to Ove Jepsen for being extremely helpful, as usual, and for pointing out an error in the manuscript. O.K.A. thanks to Guo-Qiang Liu for discussions of his spin-orbit coupled calculations. L.B. would like to thank Alessandro Toschi for many useful discussions. Finally, to all those colleagues who have published results related to ours, but of which we are not aware, we apologize for having not given reference.

This research was supported in part by the National Science Foundation under Grant No. PHY05-51164 (KITP UCSB) and by the DFG SFP1458.

\section{References}

[1] P.Y. Yu and M. Cardona, "Fundamentals of Semiconductors: Physics and Material Properties", $4^{\text {th }} E d$., SpringerBerlin Heidelberg Verlag, 2010.

[2] See for example, S. Johnston, et al.,Phys. Rev. B 82, 064513 (2010).

[3] J. Paglione and R.L. Greene, Nature Physics 6, 645 (2010).

[4] D. C. Johnston, Advances in Physics 59, 803 (2010).

[5] K. Ishida et al., J. Phys. Soc. Jpn, 78, 062001 (2009); and the special Issues of "Physica C" 469 (2009) and New Journal of Physics (11, 2009).

[6] Y. Kamihara et al., J. am. Chem Soc. 128, 10012 (2006).

[7] Already in 1996, the same compound had been found to superconduct in the group of Jeitschko, but given the low $T_{c}$ this result was not found worth a proper publication; see W. Jeitschko et al., Z. Naturforsch. 63b, 634 (2008) and refs therein.

[8] Y. Kamihara et al., J. am. Chem Soc. 130, 3296 (2008).

[9] M. Rotter et al., Phys. Rev. Lett. 101, 107006 (2008); J. H. Tapp et al., Phys. Rev. B 78, 060505 (2008); F.-C. Hsu et al., Proc. Nat. Acad. Sci. USA 105, 14262 (2008).

[10] C. Wang et al., Europhys. Lett. 83, 67006 (2008). 
[11] Other Fe-based superconductors with more complicated blocking layers have been found, mostly in Ogino's group. See for example: H. Ogino et al., Appl. Phys. Lett. 97, 072506 (2010); Appl. Phys. Expr. 3, 063103 (2010); Y. Shimizu et al., airXiv:1006.3769 (unpublished).

[12] C. de la Cruz, et al., Nature (London) 453, 899 (2008); (poly) $\mathrm{m}=0.36 \mu_{B}$; more recent reports yield larger values of the magnetic moment. See: N. Qureshi et al., arXiv:1002.4326 1 (polycrystals) $\mathrm{m}=0.63 \mu_{B}$; H.F. Li et al., arXiv: $1007.2197 \mathrm{v} 1 \mathrm{~m}=0.8 \mu_{B}$ (single + poly-crystals).

[13] C.-H. Lee, A. Iyo, H. Eisaki, H. Kito, M.T. Fernandez-Diaz, T. Ito, K. Kihou, H. Matsuhata, M. Braden, and K. Yamada, J. Phys. Soc. Jpn. 77, 083704 (2008).

[14] For a recent summary, see: Peter J. Hirschfeld and Douglas J. Scalapino Physics 3, 64 (2010); and also: I. I. Mazin, T. P. Devereaux, J. G. Analytis, Jiun-Haw Chu, I. R. Fisher, B. Muschler, R. Hackl, arXiv:1008.0032 and refs therein.

[15] L. X. Yang, et al.Phys. Rev. Lett. 102, 107002 (2009).

[16] C. Liu, T. Kondo, M. E. Tillman, R. Gordon, G. D. Samolyuk, Y. Lee, C. Martin, J. L. McChesney, S. Budko, M. A. Tanatar, E. Rotenberg, P. C. Canfield, R. Prozorov, B. N. Harmon, and A. Kaminski, arXiv:0806.2147 (unpublished).

[17] H. Ding, P. Richard, K. Nakayama, K. Sugawara, T. Arakane, Y. Sekiba, A. Takayama, S. Souma, T. Sato, T. Takahashi, Z. Wang, X. Dai, Z. Fang, G. F. Chen, J. L. Luo and N. L. Wang, Europhysics Letters, 8347001 (2008).

[18] V. B. Zabolotnyy, D. S. Inosov, D. V. Evtushinsky, A. Koitzsch, A. A. Kordyuk, G. L. Sun, J. T. Park, D. Haug, V. Hinkov, A. V. Boris, C. T. Lin, M. Knupfer, A. N. Yaresko, B. Bchner, A. Varykhalov, R. Follath, S. V. Borisenko; Nature (London) 457, 569 (2009).

[19] J. Fink, S. Thirupathaiah, R. Ovsyannikov, H. A. Drr, R. Follath, Y. Huang, S. de Jong, M. S. Golden, Yu-Zhong Zhang, H. O. Jeschke, R. Valenti, C. Felser, S. Dastjani Farahani, M. Rotter, and D. Johrendt, Phys. Rev. B 79, 155118 (2009).

[20] D. H. Lu, M. Yi, S.-K. Mo, J. G. Analytis, J.-H. Chu, A. S. Erickson, D. J. Singh, Z. Hussain, T. H. Geballe, I. R. Fisher, Z.-X. Shen, Physica C 469 452-458 (2009).

[21] M. Yi, D. H. Lu, J. G. Analytis, J.-H. Chu, S.-K. Mo, R.-H. He, X. J. Zhou, G. F. Chen, J. L. Luo, N. L. Wang, Z. Hussain, D. J. Singh, I. R. Fisher, Z.-X. Shen, Phys. Rev. B 80, 024515 (2009).

[22] D. V. Evtushinsky, D. S. Inosov, V. B. Zabolotnyy, A. Koitzsch, M. Knupfer, B. Büchner, M. S. Viazovska, G. L. Sun, V. Hinkov, A. V. Boris, C. T. Lin, B. Keimer, A. Varykhalov, A. A. Kordyuk and S. V. Borisenko Phys. Rev. B 79, 054517 (2009).

[23] S. V. Borisenko, V. B. Zabolotnyy, D. V. Evtushinsky, T. K. Kim, I. V. Morozov, A. N. Yaresko, A. A. Kordyuk, G. Behr, A. Vasiliev, R. Follath, and B. Büchner, Phys. Rev. Lett. 105, 067002 (2010).

[24] Takeshi Kondo, R. M. Fernandes, R. Khasanov, Chang Liu, A. D. Palczewski, Ni Ni, M. Shi, A. Bostwick, E. Rotenberg, J. Schmalian, S. L. Budko, P. C. Canfield, and A. Kaminski1 Phys. Rev. B 81, 060507(R) (2010).

[25] Suchitra E. Sebastian, J. Gillett, N. Harrison, P. H. C. Lau, D. J. Singh, C. H. Mielke and G. G. Lonzarich, J. Phys. Condensed Matter 20,422203 (2008).

[26] A. I. Coldea, J. D. Fletcher, A. Carrington, J. G. Analytis, A. F. Bangura, J.-H. Chu, A. S. Erickson, I. R. Fisher, N. E. Hussey, and R. D. McDonald, Phys. Rev. Lett. 101, 216402 (2008).

[27] James G. Analytis, Ross D. McDonald, Jiun-Haw Chu, Scott C. Riggs, Alimamy F. Bangura, Chris Kucharczyk, Michelle Johannes, and I. R. Fisher, Phys. Rev. B 80, 064507 (2009).

[28] J. G. Analytis, C. M. J. Andrew, A. I. Coldea, A. McCollam, J.-H. Chu, R. D. McDonald, I. R. Fisher, and A. Carrington, Phys. Rev. Lett. 103, 076401 (2009).

[29] Amalia I. Coldea, C. M. J. Andrew, J. G. Analytis, R. D. McDonald, A. F. Bangura, J.-H. Chu, I. R. Fisher, and A. Carrington, Phys. Rev. Lett. 103, 026404 (2009).

[30] M. Yi, D. H. Lu, J. G. Analytis, J.-H. Chu, S.-K. Mo, R.-H. He, M. Hashimoto, R. G. Moore, I. I. Mazin, D. J. Singh, Z. Hussain, I. R. Fisher, and Z.-X. Shen, Phys. Rev. B 80, 174510 (2009).

[31] N. Harrison and S. E. Sebastian, Phys. Rev. B 80, 224512 (2009).

[32] S. Lebegue, Phys. Rev. B 75035110 (2007).

[33] D. J. Singh and M.-H. Du, Phys. Rev. Lett. 100, 237003 (2008).

[34] D. J. Singh, Phys. Rev. B 78, 094511 (2008).

[35] Alaska Subedi, Lijun Zhang, D. J. Singh, and M. H. Du, Phys. Rev. B 78, 134514 (2008).

[36] T. Yildirim, Phys. Rev. Lett. 101, 057010 (2008).

[37] S. Ishibashi, K. Terakura, and H. Hosono, J. Phys. Soc. Jpn. 77 (2008) 053709.

[38] Z. P. Yin, et al, Phys. Rev. Lett. 101, 047001 (2008).

[39] Chao Cao, P. J. Hirschfeld, and Hai-Ping Cheng, Phys. Rev. B 77, 220506 (2008). 
[40] Verónica Vildosola, Leonid Pourovskii, Ryotaro Arita, Silke Biermann, and Antoine Georges, Phys. Rev. B 78, 064518 (2008).

[41] H. Eschrig and K. Koepernik, Phys. Rev. B 80, 104503 (2009).

[42] I. I. Mazin, D. J. Singh, M. D. Johannes, and M. H. Du, Phys. Rev. Lett. 101, 057003 (2008).

[43] K. Kuroki, Seiichiro Onari, Ryotaro Arita, Hidetomo Usui, Yukio Tanaka, Hiroshi Kontani, and Hideo Aoki, Phys. Rev. Lett. 101, 087004 (2008).

[44] L. Boeri, et al., Rev. Lett. 101, 026403 (2008) and Physica C 469, 628 (2009).

[45] L. Boeri, M. Calandra, I. I. Mazin, O. V. Dolgov, and F. Mauri, Phys. Rev. B 82, 020506 (2010).

[46] F. Yndurain, cond-mat/1009.4909 (unpublished).

[47] Q. Si and E. Abrahams, Phys. Rev. Lett. 101, 076401 (2008). P. A. Lee and X.-G. Wen, Phys. Rev. B 78, 144517 (2008). G. A. Sawatzky, I. S. Elfimov, J. van den Brink, and J. Zaanen, Europhys. Lett. 86, 17006 (2009); Mona Berciu, Ilya Elfimov, and George A. Sawatzky Phys. Rev. B 79, 214507 (2009).

[48] A. N. Yaresko, G.-Q. Liu, V. N. Antonov, and O. K. Andersen, Phys. Rev. B 79, 144421 (2009).

[49] S. Graser, G. R. Boyd, Chao Cao, Hai-Ping Cheng, P. J. Hirschfeld, and D. J. Scalapino Phys. Rev. B 77, 180514 (2008).

[50] A. V. Chubukov, D. V. Efremov, and I. Eremin Phys. Rev. B 78, 134512 (2008).

[51] L. Benfatto, et al.,Phys. Rev. B 78, 140502 (2008) and Phys. Rev. B 80, 214522 (2009).

[52] Kazuhiko Kuroki, Hidetomo Usui, Seiichiro Onari, Ryotaro Arita, and Hideo Aoki Phys. Rev. B 79, 224511 (2009).

[53] V. Cvetkovic and Z. Tesanovic, Europhys. Lett. 85, 37002 (2009).

[54] G. A. Ummarino, M. Tortello, D. Daghero, and R. S. Gonnelli, Phys. Rev. B 80, 172503 (2009).

[55] F. Wang et al., Phys. Rev. Lett. 102, 047005 (2009).

[56] Ronny Thomale, Christian Platt, Jiangping Hu, Carsten Honerkamp, and B. Andrei Bernevig, Phys. Rev. B 80, 180505 (2009).

[57] R. Thomale, C. Platt, W. Hanke, B. A. Bernevig, arXiv:1002.3599

[58] A. F. Kemper et al., New Journal of Physics 12, 073030 (2010).

[59] Hiroaki Ikeda, Ryotaro Arita, and Jan Kunes Phys. Rev. B 81, 054502 (2010).

[60] P. Popovich, A. V. Boris, O. V. Dolgov, A. A. Golubov, D. L. Sun, C. T. Lin, R. K. Kremer, and B. Keimer, Phys. Rev. Lett. 105, 027003 (2010).

[61] M. D. Johannes and I. I. Mazin, Phys. Rev. B 79, 220510 (2009).

[62] A. V. Boris, N. N. Kovaleva, S. S. A. Seo, J. S. Kim, P. Popovich, Y. Matiks, R. K. Kremer, and B. Keimer, Phys. Rev. Lett. 102, 027001 (2009); A. Charnukha, P. Popovich, Y. Matiks, D. L. Sun, C. T. Lin, A. N. Yaresko, B. Keimer, A. V. Boris, cond-mat/1009.5915; Johannes Ferber, Yu-Zhong Zhang, Harald O. Jeschke, Roser Valenti, , Phys. Rev. B 82, 165102 (2010); A. Sanna, F. Bernardini, G. Profeta, S. Sharma, J.K. Dewhurst, A. Lucarelli, L. De Giorgi, E.K. U. Gross and S. Massidda, cond-mat/1010.0220 (unpublished).

[63] I. I. Mazin, M. D. Johannes, L. Boeri, K. Koepernik, and D. J. Singh, Phys. Rev. B 78, 085104 (2008).

[64] T. Yildirim Phys. Rev. Lett. 102, 037003 (2009) and Physica C 469, 425 (2009).

[65] M. Zbiri, et al, Phys. Rev. B 79, 064511 (2009).

[66] D. Reznik, et al, Phys. Rev. B 80, 214534 (2009).

[67] Chen Fang, Hong Yao, Wei-Feng Tsai, JiangPing Hu, and Steven A. Kivelson, Phys. Rev. B 77, 224509 (2008).

[68] Fengjie Ma, Zhong-Yi Lu2, and Tao Xiang, Phys. Rev. B 78, 224517 (2008).

[69] Chi-Cheng Lee, Wei-Guo Yin, and Wei Ku, Phys. Rev. Lett. 103, 267001 (2009).

[70] S. O. Diallo, V. P. Antropov, T. G. Perring, C. Broholm, J. J. Pulikkotil, N. Ni, S. L. Budko, P. C. Canfield, A. Kreyssig, A. I. Goldman, and R. J. McQueeney, Phys. Rev. Lett. 102, 187206 (2009).

[71] E. Bascones, M. J. Caldern, and B. Valenzuela Phys. Rev. Lett. 104, 227201 (2010).

[72] P. Hansmann, R. Arita, A. Toschi, S. Sakai, G. Sangiovanni, and K. Held, Phys. Rev. Lett. 104, 197002 (2010).

[73] Z. P. Yin, K. Haule, G. Kotliar, arXiv:1007.2867

[74] Markus Aichhorn, Leonid Pourovskii, Veronica Vildosola, Michel Ferrero, Olivier Parcollet, Takashi Miyake, Antoine Georges, and Silke Biermann, Phys. Rev. B 80, 085101 (2009); Markus Aichhorn, Silke Biermann, Takashi Miyake, Antoine Georges, and Masatoshi Imada, Phys. Rev. B 82, 064504 (2010).

[75] S. L. Skornyakov, A. V. Efremov, N. A. Skorikov, M. A. Korotin, Yu. A. Izyumov, V. I. Anisimov, A. V. Kozhevnikov, and D. Vollhardt, Phys. Rev. B 80, 092501 (2009).

[76] O. K. Andersen and T. Saha-Dasgupta, Phys. Rev. B 62, R16219 (2000).

[77] O. K. Andersen and L. Boeri, to appear in the New Journal of Physics (2011). See (hear) also: http://online.itp.ucsb.edu/online/highertc09/andersen/

[78] Ying Ran, Fa Wang, Hui Zhai, Ashvin Vishwanath, and Dung-Hai Lee, Phys. Rev. B 79, 014505 (2009).

[79] P. Richard, K. Nakayama, T. Sato, M. Neupane, Y.-M. Xu, J. H. Bowen, G. F. Chen, J. L. Luo, N. L. Wang, X. Dai, Z. Fang, H. Ding, and T. Takahashi, Phys. Rev. Lett. 104, 137001 (2010). 
[80] P. Blaha et al.,http://www.wien2k.at

[81] O. K. Andersen, Phys. Rev. B 12, 3060 (1975).

[82] A. I. Goldman, D. N. Argyriou, B. Ouladdiaf, T. Chatterji, A. Kreyssig, S. Nandi, N. Ni, S. L. Budko, P. C. Canfield, and R. J. McQueeney Phys. Rev. B 78, 100506 (2008).

[83] P.C. Canfield et al., Physica C 469, 404 (2009).

[84] A. Kreyssig, M. A. Green, Y. Lee, G. D. Samolyuk, P. Zajdel, J. W. Lynn, S. L. Budko, M. S. Torikachvili, N. Ni, S. Nandi, J. B. Leao, S. J. Poulton, D. N. Argyriou, B. N. Harmon, R. J. McQueeney, P. C. Canfield, and A. I. Goldman, Phys. Rev. B 78, 184517 (2008).

[85] Lijun Zhang and D. J. Singh, Phys. Rev. B 79, 174530 (2009).

[86] O. Gunnarsson, J. Phys. F: Metal Phys. 6, 587 (1976).

[87] O. K. Andersen, J. Madsen, U.K. Poulsen, O. Jepsen and J. Kollar: "Magnetic ground state properties of transition metals". Physica B 86-88, 249 (1977).

[88] L. M. Sandratskii, J. Phys. Condensed Matter 38565 (1991).

[89] S Lebgue, Z P Yin and W E Pickett, New Journal of Physics 11, 025004 (2009).

[90] H. Wadati, I. Elfimov, and G. A. Sawatzky, Phys. Rev. Lett. 105, 157004 (2010).

[91] M. Zwierzycki and O. K. Andersen Acta Physica Polonica A 115, 64 (2009). 